\title{
Impact of Optical Transmission on Multi-band OFDM \\ Ultra-Wideband Wireless System with Fiber Distribution
}

\author{
Meer Nazmus Sakib
}

\author{
A Thesis \\ In the Department \\ of
}

Electrical and Computer Engineering

\begin{abstract}
Presented in Partial Fulfillment of the Requirements
For the Degree of Master of Applied Science at

Concordia University

Montréal, Québec, Canada
\end{abstract}

April 2009

(C) Meer Nazmus Sakib, 2009 


$\begin{array}{ll}\begin{array}{l}\text { Library and Archives } \\ \text { Canada }\end{array} & \begin{array}{l}\text { Bibliothèque et } \\ \text { Archives Canada }\end{array} \\ \begin{array}{l}\text { Published Heritage } \\ \text { Branch }\end{array} & \begin{array}{l}\text { Direction du } \\ \text { Patrimoine de l'édition }\end{array} \\ \begin{array}{l}\text { 395 Wellington Street } \\ \text { Ottawa ON K1A ON4 } \\ \text { Canada }\end{array} & \begin{array}{l}395, \text { rue Wellington } \\ \text { Ottawa ON K1A ON4 } \\ \text { Canada }\end{array}\end{array}$

Your file Votre référence

ISBN: 978-0-494-63303-8

Our file Notre reférence

ISBN: 978-0-494-63303-8

NOTICE:

The author has granted a nonexclusive license allowing Library and Archives Canada to reproduce, publish, archive, preserve, conserve, communicate to the public by telecommunication or on the Internet, loan, distribute and sell theses worldwide, for commercial or noncommercial purposes, in microform, paper, electronic and/or any other formats.

The author retains copyright ownership and moral rights in this thesis. Neither the thesis nor substantial extracts from it may be printed or otherwise reproduced without the author's permission.

\begin{abstract}
AVIS:
L'auteur a accordé une licence non exclusive permettant à la Bibliothèque et Archives Canada de reproduire, publier, archiver, sauvegarder, conserver, transmettre au public par télécommunication ou par l'Internet, prêter, distribuer et vendre des thèses partout dans le monde, à des fins commerciales ou autres, sur support microforme, papier, électronique et/ou autres formats.
\end{abstract}

L'auteur conserve la propriété du droit d'auteur et des droits moraux qui protège cette thèse. $\mathrm{Ni}$ la thèse ni des extraits substantiels de celle-ci ne doivent être imprimés ou autrement reproduits sans son autorisation.
In compliance with the Canadian Privacy Act some supporting forms may have been removed from this thesis.

While these forms may be included in the document page count, their removal does not represent any loss of content from the thesis.
Conformément à la loi canadienne sur la protection de la vie privée, quelques formulaires secondaires ont été enlevés de cette thèse.

Bien que ces formulaires aient inclus dans la pagination, il n'y aura aucun contenu manquant.

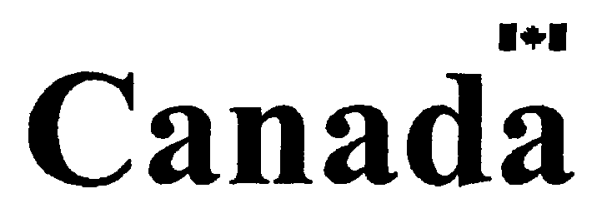




\title{
ABSTRACT \\ Impact of Optical Transmission on Multiband OFDM Ultra-Wideband Wireless System with Fiber Distribution
}

\begin{abstract}
Meer Nazmus Sakib
The performance of MB-OFDM UWB over fiber transmission system is investigated considering optical modulation and demodulation impact. Theoretical analysis of the effect of fiber dispersion, optical transmitter and optical receiver response on system performance is carried out considering amplitude and phase distortion. Experiments are conducted and verified by our theoretical analysis and good agreement is obtained. It is found that RF modulation index of $\sim 4 \%$ is optimum for optical transmitter with Mach-Zehnder modulator, and optical receiver with Chebyshev-II response is the best for MB-OFDM UWB over fiber. It is also found that high received optical power is required for transmission of MB-OFDM UWB signal over fiber.
\end{abstract}

Theoretical analysis of the effect of fiber chromatic dispersion induced laser phase to intensity noise or relative intensity noise (RIN) on system performance is carried out. Experiments are also conducted to verify our theoretical analysis. Simulation is also carried out to show the relationship between RIN and center frequency of UWB bands. It is found that the parameters like laser output power, laser linewidth and fiber dispersion that control RIN, will critically affect the overall performance of a UWB over fiber system.

The performance of MB-OFDM UWB over fiber transmission system is also studied considering the effect of in-band jammers such as WiMAX, WLAN MIMO, 
WLAN and marine radar. Experiments were performed to show the effect of fiber transmission under various interferer power levels. It is found that in-band interferers can cause severe degradation in system performance if certain interferer to UWB peak power ratio is not maintained. 


\section{Acknowledgements}

I would like to express my sincere gratitude to my supervisor Dr. X. Zhang for his guidance, advice and financial support for me to finish this dissertation.

I thank Bouchaib Hraimel, for his support and help in my experiment and also Mohmoud Mohamed and Wei Jiang, for their suggestions this thesis.

I have sincere appreciation to the Fonds de recherché sur la nature et les technologies (FQRNT), Quebec, Canada for supporting this research project.

I would like to thank Adi Levi from WisAir for providing technical information about the UWB transmitter/ receiver module. I am grateful to Luay Thomas from TMetrix, Richard Dhummel from Tektronix and Genevieve Landry from Agilent Technologies for the expensive LeCroy, Tektronix and Agilent data analyzers, respectively. Also thanks to Dr. C.W. Trueman and Dr. Don Davis for helping me with the vector network analyzer.

Last but not least, I am very grateful to my father and mother for their love and support throughout my life. 


\section{Table of Contents}

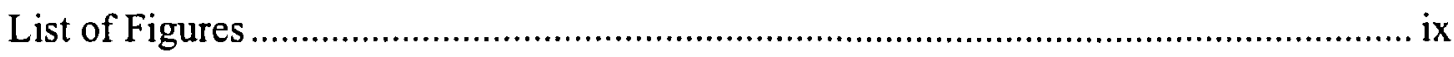

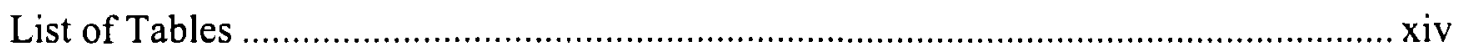

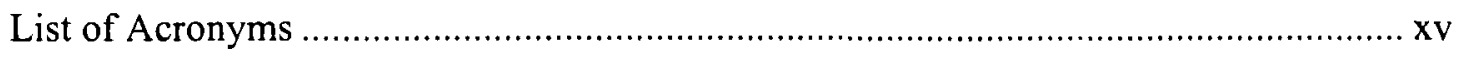

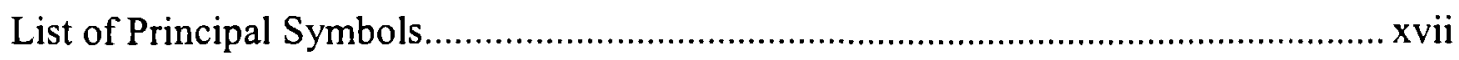

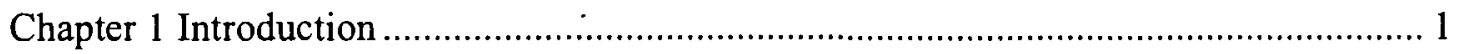

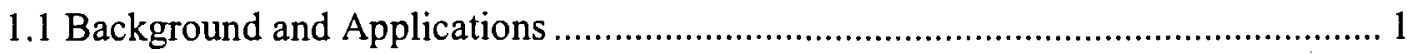

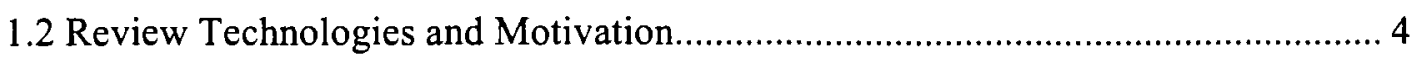

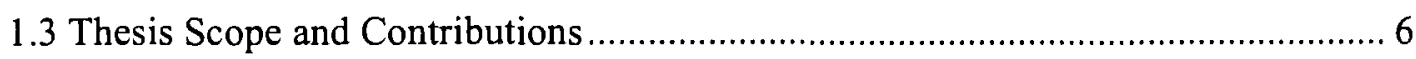

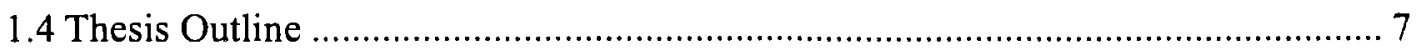

Chapter 2 Radio over Fiber Technologies ........................................................... 10

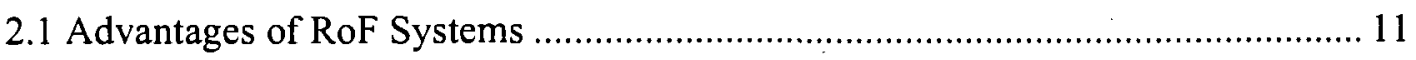

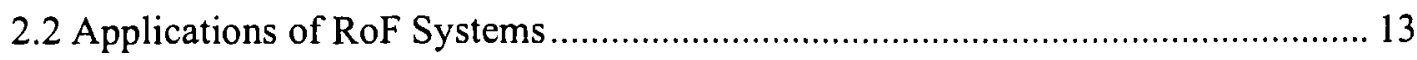

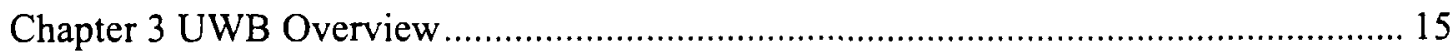

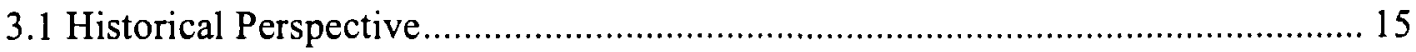

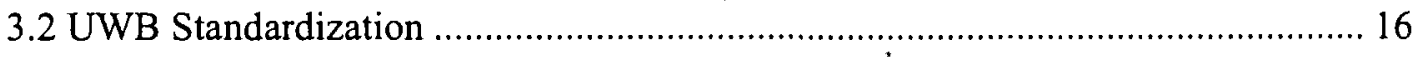

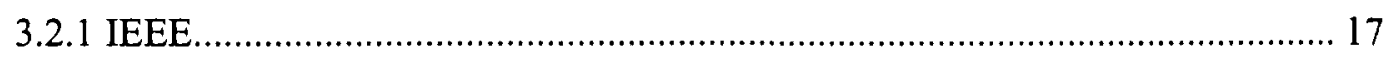

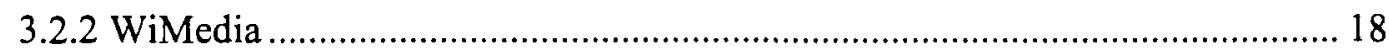

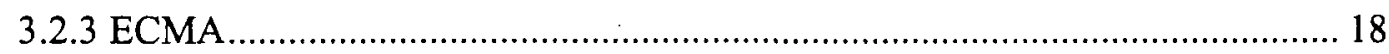

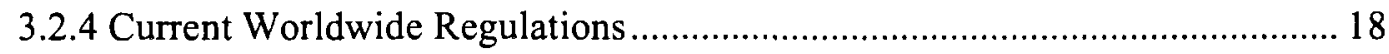

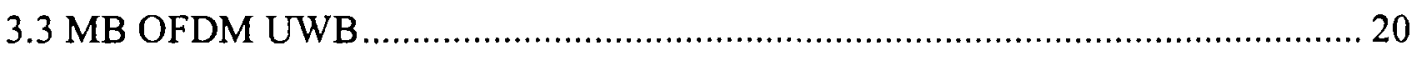

3.3.1 MB OFDM UWB Wireless Transmitter and Receiver............................ 21 
3.3.2 Timing and Synchronization Parameters .................................................. 24

3.3.3 Modes of Operation ............................................................................ 26

3.3.4 Data Rate Dependent Parameters................................................................ 29

3.4 Mathematical Framework for MB OFDM UWB............................................ 31

3.5 UWB over Fiber Technologies …………………......................................... 32

Chapter 4 Experimental Setups and Theoretical analysis............................................... 33

4.1 Experimental System Configuration for UWB over Fiber System.......................... 33

4.1.1 Single Channel UWB over Fiber System .......................................................... 33

4.1.2 MB-OFDM UWB over Fiber System and In-band Interferers........................ 36

4.2 Measures of performance: EVM versus PER …………...................................... 40

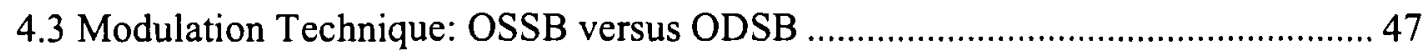

4.4 Calculation of EVM for transmission through optical fiber using DE-MZM......... 50

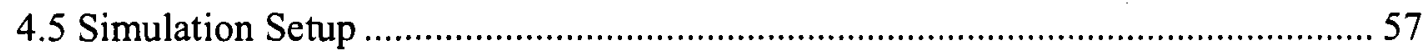

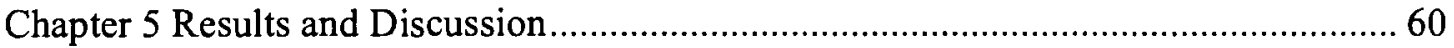

5.1 Impact of optical Modulation and Fiber Transmission ............................................ 61

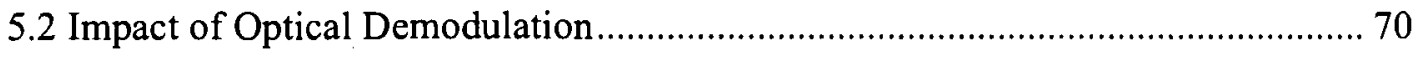

5.3 Impact of Received Optical Power................................................................... 77

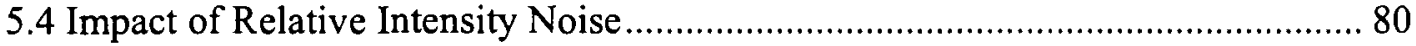

5.4.1 Laser Intrinsic RIN, Resonant Frequency and Laser Output Power ................ 80

5.4.2 Laser Linewidth, Fiber Dispersion and RF frequency .................................. 84

5.5 Performance of Multi-band OFDM Ultra-Wideband over Fiber Transmission under

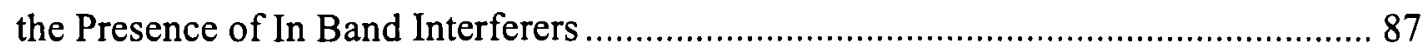

5.5.1 UWB over Single Mode Fiber without Interferers .......................................... 89 
5.5.2 Performance of Band Group 1 of MB OFDM UWB under the presence of

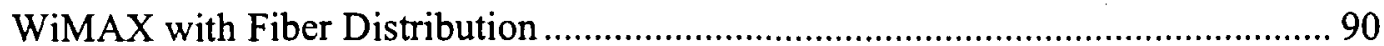

5.5.3 Performance of Band Group 2 of MB OFDM UWB under the presence of

WLAN MIMO and WLAN with Fiber Distribution 92

5.5.3 Performance of Band Group 4 of MB OFDM UWB under the presence of Marine Radar with Fiber Distribution 96

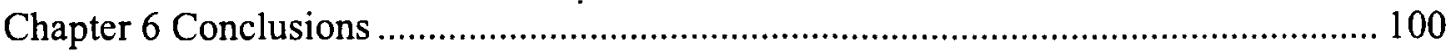

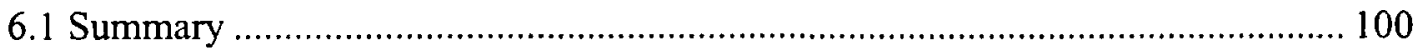

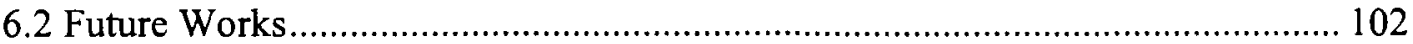

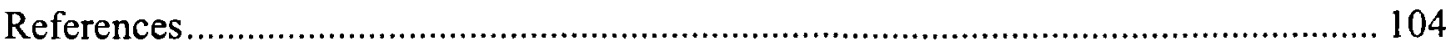

Appendix A Analysis of Optical Receiver Noise ................................................ 109

Appendix B Simulated magnitude and Delay Response of Chebyshev-II Filter............ 113 


\section{List of Figures}

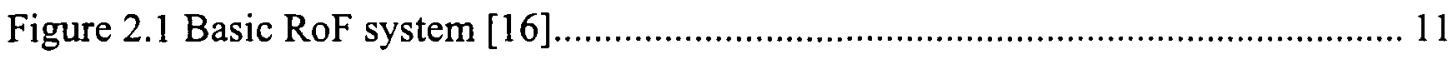

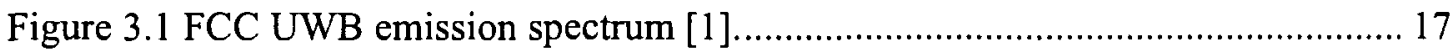

Figure 3.2 Current worldwide regulation for UWB [21] .......................................... 19

Figure 3.3 First three channels of MB-OFDM UWB wireless in (a) frequency domain, (b)

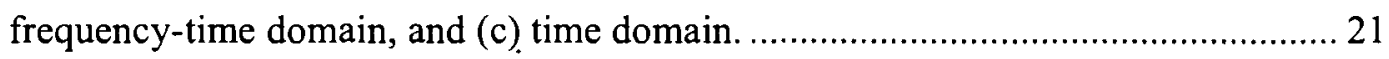

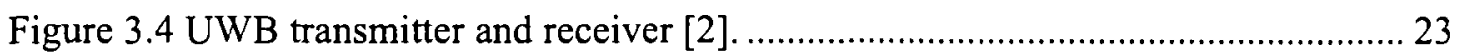

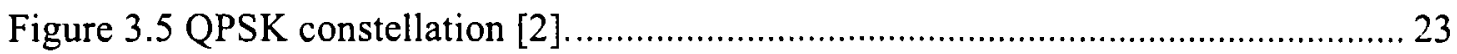

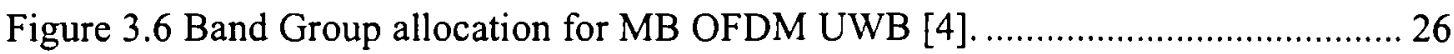

Figure 4.1 Experimental setup for externally modulated MB-OFDM UWB over fiber

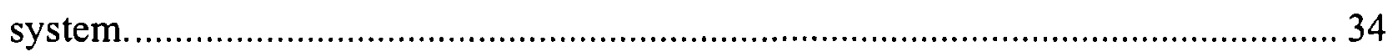

Figure 4.2 Photograph of experimental setup for externally modulated MB-OFDM UWB

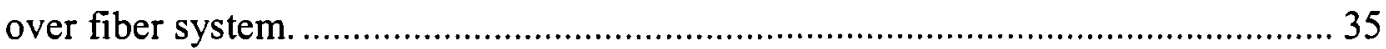

Figure 4.3 Experimental setup for externally modulated MB-OFDM UWB over fiber system under the presence of in-band interferers (NF: noise figure, G: Gain, R: responsivity). 37

Figure 4.4 PER versus UWB power and data rate for $20 \mathrm{~km}$ optical link and $1 \mathrm{~m}$ wireless link (dotted line corresponds to a PER of $8 \%$ )

Figure 4.5 PER versus wireless range and data rate for $20 \mathrm{~km}$ of optical link.

Figure 4.6 Measured optical signal (a) OSSB and (b) ODSB. 48

Figure 4.7 Measured EVM using OSSB and ODSB. 49

Figure 4.8 QPSK OFDM constellation in a) two dimension b) three dimension received at the real time oscilloscope for band group 1 after $20 \mathrm{Km}$ of fiber transmission with bit 
rate of $200 \mathrm{Mb} / \mathrm{s}$ (Colors indicate intensity of power concentration in the received symbol) 50

Figure 4.9 Simulation setup for MB OFDM UWB (OSA: optical spectrum analyzer, RSA: RF spectrum analyzer) 58

Figure 5.1 Spectral mask test for UWB. 60

Figure 5.2 Measured EVM with RF modulation index with a parameter of fiber length for bit rate of (a) $53.3 \mathrm{Mb} / \mathrm{s}$ and (b) $200 \mathrm{Mb} / \mathrm{s}$. 63

Figure 5.3 Relative amplitude of subcarriers in second band versus RF modulation index for back-to-back UWB over fiber. 63

Figure 5.4 Calculated relative amplitude of subcarriers at 1, 32, 64, 96 and 128 in band 2 versus RF modulation index for UWB over fiber at (a) 20 and (b) $40 \mathrm{~km}$ of fiber... 65

Figure 5.5 Calculated phase distortion of subcarriers (1,32, 64, 96 and 128) in band-two versus RF modulation index at (a) 20 and (b) $40 \mathrm{~km}$ of fiber transmission. 67

Figure 5.6 Calculated EVM degradation versus fiber length with respect to back to back and $0 \mathrm{dBm}$ received optical power. Black square: experimental results for 20,40 and $52 \mathrm{~km}$. 68

Figure 5.7(a) Measured magnitude $\left|S_{21}\right|$ and (b) measured Phase response of the experimental filter measured with a HP 8720 vector network analyzer. 71

Figure 5.8 Measured (symbol) and simulated (line) EVM using two receivers. Black square: experimental results using optical $\mathrm{Rx}$ with Chebyshev-I response, Black circle: experimental results using the "ideal" optical Rx. 72

Figure 5.9 Simulated EVM using optical receiver with different responses. 72 
Figure 5.10 Simulated EVM with Chebyshev-II filter order and bandwidth for $200 \mathrm{Mb} / \mathrm{s}$ UWB signal transmitted over $20 \mathrm{~km}$ of fiber. 75

Figure 5.11 Measured EVM versus received optical power at photodetector. 78

Figure 5.12 Calculated EVM degradation versus received optical power for back-to-back transmission with respect to $0 \mathrm{dBm}$ received optical power. 79

Figure 5.13 Measured spectral density of RIN as a function of frequency for back to back transmission. 82

Figure 5.14 Measured RIN peak frequency and corresponding spectral density of RIN for back to back transmission. 83

Figure 5.15 Measured EVM with laser output power for back to back transmission at bit rate of $200 \mathrm{Mb} / \mathrm{s}$. 83

Figure 5.16 Calculated RIN versus frequency for 20,40 and $60 \mathrm{~km}$ (Solid: linewidth of $30 \mathrm{MHz}$. Dotted: linewidth of $1 \mathrm{GHz}$ ). 84

Figure 5.17 Calculated EVM degradation versus fiber length with respect to back-toback. Square: experimental results for 20,40 and $52 \mathrm{~km}$. 85

Figure 5.18 Simulated (line) EVM versus bands in an MB UWB over fiber system. Square: experimental results for 0,20 and $40 \mathrm{~km}$ centered at $3.432,3.96$ and 4.488 $\mathrm{GHz}$ band...... 86

Figure 5.19 Spectrum of UWB signal with narrow band interferes. 88

Figure 5.20 RF spectrum of UWB band group 1 and WiMAX (a) transmitted at point A (b) received at point $F$ in Fig. 2 for bit rate of $200 \mathrm{Mb} / \mathrm{s}$ with $20 \mathrm{~km}$ fiber transmission (Interferer to UWB peak power ratio is $20 \mathrm{~dB}$ ). 91 
Figure 5.21 EVM performance of UWB over fiber transmission under the presence of WiMAX as a function of WiMAX to UWB peak power ratio (Solid lines: best fitted curves, dotted lines: without interference).

Figure 5.22 RF spectrum of UWB band group 1 and WLAN MIMO (a) transmitted at point $A(b)$ received at point $F$ in Fig. 2 for bit rate of $200 \mathrm{Mb} / \mathrm{s}$ with $20 \mathrm{~km}$ fiber transmission (Interferer to UWB peak power ratio is $20 \mathrm{~dB}$ ). 93

Figure 5.23 EVM performance of UWB over fiber transmission under the presence of WLAN MIMO as a function of WLAN MIMO to UWB peak power ratio (Solid lines: best fitted curves, dotted lines: without interference) 94

Figure 5.24 RF spectrum of UWB band group 2 and WLAN (a) transmitted at point A (b) received at point $\mathrm{F}$ in Fig. 2. for bit rate of $200 \mathrm{Mb} / \mathrm{s}$ with $20 \mathrm{~km}$ fiber transmission (Interferer to UWB peak power ratio is $20 \mathrm{~dB}$ ). 95

Figure 5.25 EVM performance of UWB over fiber transmission under the presence of WLAN as a function of WLAN to UWB peak power ratio (Solid lines: best fitted curves, dotted lines: without interference). 96

Figure 5.26 RF spectrum of UWB band group 4 and marine radar (a) transmitted at point A (b) received at point $F$ in Fig. 2 for bit rate of $200 \mathrm{Mb} / \mathrm{s}$ with $20 \mathrm{~km}$ fiber transmission (Interferer to UWB peak power ratio is $20 \mathrm{~dB}$ ) 97

Figure 5.27 EVM performance of UWB over fiber transmission under the presence of WLAN as a function of WLAN to UWB peak power ratio (Solid lines: best fitted curves, dotted lines: without interference). 98

Figure 5.28 Received time domain spectrum for band group 4 after $20 \mathrm{Km}$ of fiber transmission with bit rate of $200 \mathrm{Mb} / \mathrm{s}$ without any signal interferer. 98 
Figure 5.29 Received time domain spectrum for band group 4 after $20 \mathrm{Km}$ of fiber transmission with bit rate of $200 \mathrm{Mb} / \mathrm{s}$ with radar signal as interferer (Interferer to UWB peak power ratio is $20 \mathrm{~dB}$ ).

Figure A.1 Noise power level at the receiver versus received optical power for back-toback transmission. 111

Figure A.2 Calculated total RIN versus fiber length. 111

Figure B.1 Simulated (a) magnitude and (b) delay response of Chebyshev-II filter...... 114 


\section{List of Tables}

Table 2.1: Comparison between SMF and coaxial cable

Table 3.1: QPSK Encoding Table [2]

Table 3.2: Timing Related Parameters of MB OFDM [2] .25

Table 3.3: Band Group allocation for MB OFDM UWB [4].... .26

Table 3.4: Time frequency codes for Group-1 MB OFDM system [4] .28

Table 3.5: Data rate dependent parameters MB OFDM system [4] .29

Table 3.6: Sensitivity of UWB receiver [4] .30

Table 3.7: Permissible Relative Constellation Error (EVM) 30

Table 4.1: Generated UWB Sub Bands and Corresponding Interferers. 38

Table 4.2: Generated UWB Sub-Bands, Interferers and Their Corresponding PSD......38

Table 5.1: Measured EVM Performance of UWB Transmission without interferers......90 


\section{List of Acronyms}

\begin{tabular}{|c|c|}
\hline ACPR & Adjacent Channel Power \\
\hline ASE & Amplified Spontaneous Emission \\
\hline $\mathrm{AM} / \mathrm{AM}$ & Amplitude/ Amplitude \\
\hline $\mathrm{AM} / \mathrm{PM}$ & Amplitude/ Phase \\
\hline BPSK & Binary Phase Shift Keying \\
\hline DCF & Dispersion Compensating Fiber \\
\hline $\mathrm{DE}$ & Dual Electrode \\
\hline DFB & Distributed Feedback \\
\hline $\mathrm{CW}$ & Continuous Wave \\
\hline EBF & Electrical Bandpass Filter \\
\hline ECMA & European Computer Manufacturers' Association \\
\hline EDFA & Erbium Doped Fiber Amplifier \\
\hline EIRP & Effective Isotropic Radiated Power \\
\hline EVM & Error Vector Magnitude \\
\hline FCC & Federal Communications Commission \\
\hline FFT & Fast Fourier Transform \\
\hline GVD & Group Velocity Dispersion \\
\hline IFFT & Inverse Fast Fourier Transform \\
\hline $\mathrm{MAC}$ & Multiple Access \\
\hline MB & Multi-Band \\
\hline MMF & Multi Mode Fiber \\
\hline MZM & Mach-Zehnder Modulator \\
\hline
\end{tabular}




$\begin{array}{ll}\text { ODSB } & \text { Optical Double Side Band } \\ \text { OFDM } & \text { Orthogonal Frequency Division Multiplexing } \\ \text { OSSB } & \text { Optical Single Side Band } \\ \text { OSA } & \text { Optical Spectrum Analyzer } \\ \text { PER } & \text { Packet Error Rate } \\ \text { PHY } & \text { Physical Layer } \\ \text { QAM } & \text { Qudrature Amplitude Modulation } \\ \text { QPSK } & \text { Quadrature Phase Shift keying } \\ \text { QoS } & \text { Quality of Service } \\ \text { RF } & \text { Radio Frequency } \\ \text { RIN } & \text { Relative Intensity Noise } \\ \text { RoF } & \text { Radio over Fiber } \\ \text { SMF } & \text { Single Mode Fiber } \\ \text { VNR } & \text { Signal to Noise Ratio } \\ \text { TFC } & \text { Time Frequency Code } \\ \text { UWB } & \text { Ultra wideband } \\ \text { WLAN } & \text { Wireless Access Networks } \\ \text { VA } & \text { Variable Attenuator } \\ \text { VOriable Optical Attenuator } \\ \text { OAvity Surface Emitting Laser }\end{array}$




\section{List of Principal Symbols}
A
Ampere
D
Dispersion Parameter $(\mathrm{ps} /(\mathrm{nm} \cdot \mathrm{km}))$
$D_{M}$
Material Dispersion $(p s /(n m \cdot k m))$
$D_{w}$
Waveguide Dispersion ( $p s /(\mathrm{nm} \cdot \mathrm{km}))$
$f_{c} \quad$ RF carrier frequency
$\hbar$
The Reduced Planck Constant $\left(6.582 \times 10^{-16} \mathrm{eV} \cdot \mathrm{s}\right)$
$L_{\text {eff }} \quad$ Effective Interaction Length $(\mathbf{k m})$
$N_{S T} \quad$ Number of subcarriers
$s$
Dispersion Slope $\left(\mathrm{ps} /\left(\mathrm{nm}^{2} \cdot \mathrm{km}\right)\right)$
$T_{C P} \quad$ Cyclic prefix (ns)
$T_{G I} \quad$ Guard Interval
$\lambda \quad$ Wavelength (nm)
$\omega \quad$ Frequency $\left(T H_{z}\right)$
$\beta \quad$ Propagation Constant (radians/meter)
$\alpha \quad$ Fiber loss $(d B / k m)$ 
$\beta_{2} \quad$ GVD Coefficient $\left(p s^{2} / \mathrm{km}\right)$

$\Delta f \quad$ Subcarrier frequency spacing $(\mathrm{MHz})$ 


\section{CHAPTER 1 INTRODUCTION}

\subsection{Background and Applications}

The basic concept of ultra wideband (UWB) is to use ultra short pulse $(<2 \mathrm{~ns})$ in time domain to spread the frequency energy over wide bandwidth (>500MHz) to a low level, in order to share the spectrum with existing narrowband transmission without causing unwanted interference. The US Federal Communications Commission (FCC) was the first to open radio spectrum of 3.1-10.6 GHz for UWB use [1]. The wide personal area network (WPAN) working group of IEEE responded by the draft $802.15 .3 \mathrm{a}$ standard, which divides the whole spectrum into 14 bands with bandwidth of $528 \mathrm{MHz}$ for each band [2]. The 14 channels are organized in five groups. Each group has three channels except group five which has only two channels. A variable throughput from 53.3 to $480 \mathrm{Mb} / \mathrm{s}$ in each channel is suggested. To enable operation of multiple UWB systems at the same time, the carrier hops around in frequency. The carrier can hop to one of fourteen channels $(2904+528 \mathrm{n} \mathrm{MHz}, \mathrm{n}=1,2 \ldots 14)$. Other countries quickly followed the FCC and IEEE initiative. Though in Japan, Korea, China and European Union countries, transmission in band group two $(4.752-6.336 \mathrm{GHz})$ is not permitted to avoid interference with existing IEEE 802.11a WLAN. The greatest advantage with UWB radio is that it is software configurable, so any of the frequency bands can be turned off to meet specific spectral requirements.

However, the WiMedia alliance was the first to take major initiative towards the implementation by selecting multi-band (MB) orthogonal frequency division 
multiplexing (OFDM) for high speed UWB wireless [3]. In December 2007, European Computer Manufacturers' Association (ECMA) adopted the WiMedia approach and ratified ECMA-368 standard [4] which gave huge boost for the industry and academia. Various prototypes of WiMedia UWB devices are already in the market. Low cost UWB devices are expected to hit the mass market by early 2012 .

UWB technology shows a lot of potential. It has many important applications. These include the following:

- Broad Band Wireless Access Networks - The most important use of UWB is in the field of broadband wireless local access networks (WLANs). UWB devices can support bit rates of up to $480 \mathrm{Mb} / \mathrm{s}$ compared to $54 \mathrm{Mb} / \mathrm{s}$ in IEEE 802.11 WLAN and $3 \mathrm{Mb} / \mathrm{s}$ in Bluetooth, respectively. IEEE 802.11 requires high power so it cannot be used in portable handheld devices. Using Bluetooth it takes about 45 minutes to transfer data from a $1 \mathrm{~Gb}$ memory card whereas it takes about only 3 minutes to transfer the same data if UWB is used. Sony and Hitachi already included UWB interfaces with their new DVD cameras. It is thought that small UWB device will soon replace USB pen drives, garage door openers, etc.

- Stealth - In order to satisfy the effective isotropic radiated power (EIRP) requirement defined by FCC, UWB signals are designed to have noise-like properties and the energy of the high frequency signal is spread over a very large 
bandwidth. As a result the signal appears to be very low level background noise to an unintended narrowband receiver.

- Sensor Application - UWB can transfer data over a high bit rate without causing interference to other narrowband systems. Especially in the field of medical sensors UWB can replace wires connected to a patient's body and improve comfort.

- Position Location - UWB systems are capable of determining the 3D location of any of its transponders to within a few centimeters.

- Radar Imaging - UWB systems can be used as an open-air through-wall or ground-penetrating radar imager. In several airports of Denmark and Netherlands UWB radar imaging systems are used for searching guns and explosives. Also UWB's ability to penetrate through walls makes it perfect for use in cubicles under office environments.

- Vehicular Radar Systems - UWB also has application in vehicular radar systems for use in collision avoidance and parking aids.

Despite of its promises UWB has a serious limitation in terms of transmission coverage. Due to very low transmitted power, it is impossible to distribute UWB signals over air for more than tens of meter. On the other hand, due to the wide bandwidth of 7.5 
$\mathrm{GHz}$ it is very hard and expensive to distribute UWB radio signals over coaxial cable. Optical fiber is well known for its low loss, low cost and wide bandwidth in addition to light weight and mature technology. Therefore it will be very promising using UWB radios over fiber technique for UWB distribution.

\subsection{Review Technologies and Motivation}

UWB over fiber is a fast emerging technology. The field is relatively new and the total number of publications on UWB over fiber will not be more than fifteen. Y. Guennec, et al, first presented the idea of transmission of MB OFDM UWB over fiber in an invited paper at the Optical Fiber Communication Conference and Exposition (OFC), 2006 that reflected French and Orange Telecom's contemporary research and development in this field [5]. In the same conference A. Pizzinat, et al, showed that the direct laser modulation can be used to transmit UWB signals over single mode fiber (SMF), and it was found that direct modulation is limited by laser bandwidth, linewidth and stability [6]. Distribution of UWB wireless over SMF was also studied by M. Yee, et al [7]. In early 2007 the feasibility of using a low bandwidth multimode fiber to transmit $3 \times 640 \mathrm{Mb} / \mathrm{s}$ MB OFDM UWB radio signals with pre-distortion over a distance of $500 \mathrm{~m}$ by using low cost transmitter optical subassembly (TOSA) and receiver optical subassembly (ROSA) [8]. In September 2007 Y. Guo, et al, presented the packet error rate performance (PER) of UWB over fiber systems when transmitted over multimode

fiber [9]. Y. Ben-Ezra, et al, showed the combined wireless and optical fiber transmission of MB OFDM UWB by directly modulating $4.8 \mathrm{GHz}$ vertical cavity surface emitting 
lasers (VCSELs) [10]. The results showed that transmission of UWB signals over multimode fiber (MMF) is limited to several hundred meters and it was demonstrated that frequency bands of UWB higher than $4.5 \mathrm{GHz}$ are not suitable for transmission over MMF. In an article published in IEEE International Microwave Symposium in May 2008, M. Lee, et al, investigated the performance of a WLAN over fiber system due to band group $1(3.168 \sim 4.752 \mathrm{GHz})$ of UWB and it was found that Band Group 1 will not interfere with WLAN at 2.4 and $5 \mathrm{GHz}$ since frequency spectrum of WLAN at that frequency is far away [11]. All these experiments were performed by directly modulating a laser.

To have UWB wireless coverage of larger than $20 \mathrm{~km}$, similar to fiber-to-the home (FTTH) applications, it was proposed to use externally modulated UWB over fiber [12]. Recently, transmission UWB signals over SMF using external and direct modulation was examined and compared between impulse and MB OFDM UWB [13]. Bidirectional transmission of UWB over fiber was also studied by D. Smith, et al, using a reflective electro-absorption transducer using two different optical wavelengths of 1310 and $1550 \mathrm{~nm}$ for downstream and upstream transmission, respectively [14]. Apart from these work on physical (PHY) layer A. pizzinat, et al, also proposed several multiple access (MAC) layer architectures for distribution of MB OFDM UWB wireless over fiber [15]. However, there are many areas of UWB over fiber transmission that is yet to be covered.

Motivated by the previous researchers the aims of this thesis are to:

- Introduce different UWB over fiber case scenarios, system and novel device concepts. 
- Provide detailed performance analysis of UWB over fiber applications with different optical modulation schemes to select the best technique for UWB radio over optical fiber distribution.

- Focus on the low-cost and high performance transmission UWB over fiber for mass application.

- Identify future research areas related to the design and performance of UWB over fiber.

\subsection{Thesis Scope and Contributions}

In this thesis for the first time to our knowledge, the combined effect of fiber dispersion, and nonlinearities of optical transmitter's and optical receiver's response on the OFDM subcarriers for MB OFDM UWB over fiber system is investigated through detailed theoretical and experimental analysis. The effect of optical receiver response, noise and received optical power at the photodetector is investigated in an MB-OFDM UWB over fiber system. The main contributions of this thesis are:

1. The performance of MB OFDM UWB over fiber transmission system is investigated in details. Theoretical analysis of the effect of fiber dispersion, optical transmitter and optical receiver response on system performance is carried out considering amplitude and phase distortion. Experiments are conducted and verified by our theoretical analysis and good agreement is obtained. 
2. The response of the optical receiver is studied in terms of amplitude and phase distortion. The effect of receiver bandwidth on system performance also investigated.

3. The effect of received optical power on system performance and optical receiver noise is examined. High received optical power requirement of MB UWB over fiber system is elucidated.

4. The effect of fiber chromatic dispersion induced laser phase to intensity noise conversion or relative intensity noise (RIN) on performance is investigated in terms of laser intrinsic RIN, laser linewidth, fiber dispersion and carrier frequency.

5. The performance of MB-OFDM UWB over fiber transmission system is investigated considering the effect of in-band narrowband jammers such as WIMAX, WLAN MIMO, WLAN and marine radar.

\subsection{Thesis Outline}

The objective of this research work is to develop a UWB over fiber system that will be ensure best performance and will provide economic solution to this fast growing technology. The rest of the thesis is organized as follows. 
Chapter 2 introduces the basic operation of a radio over fiber system, its advantages and real world applications.

Chapter 3 presents an overview of UWB wireless technology. This chapter focuses on key aspects of MB OFDM UWB with focus on IEEE 802.15.3 draft and ECMA-368 standard. The current frequency bands and standardization issues are also discussed. Basic mathematical framework and physical layer structure is described.

Chapter 4 presents experimental setup for single channel MB OFDM UWB over fiber systems. Then the experimental setup for UWB over fiber transmission with multiple narrow band interferers is depicted. The chapter starts with a relative comparison between PER and error vector magnitude (EVM) as measures of system performance. Optical single sideband (OSSB) and optical double sideband (ODSB) are compared, as commonly used optical external modulation techniques. Then theoretical analyses are given for single channel externally modulated UWB over fibers system considering amplitude and phase distortion experienced by each of the OFDM subcarriers within one symbol. At the end of the chapter the simulation setup for UWB over fiber transmission using a commercial software VPI Transmission maker is presented.

Chapter 5 the performance of UWB over fiber system is clarified with focus on the ECMA-368 standard using MB OFDM OFDM. Optical transmitter's nonlinearities and fiber dispersion effect on system performance are studied experimentally and compared to the theoretical analysis. Simulations and experiments are performed to 
assess the effect of optical receiver's response on UWB performance. In addition, received optical power requirement in the UWB over fiber system, which was not clarified in [12], is elucidated. Also, the effect of RIN is discussed through simulations and experiments in details. Then the performance degradation of a MB-OFDM UWB over fiber is presented under the presence of in-band interferers, i.e. WiMAX, WLAN MIMO, WLAN and radar signals.

Chapter 6 concludes the thesis with the amount of progress that was accomplished and potential implication to knowledge, theory and practice. Also discussed is the future work that remains and over all insights. 


\section{Chapter 2 Radio over Fiber Technologies}

In RoF the light is modulated by a high frequency RF signal and transmitted over optical fiber. RoF technology was developed by US Defense Advanced Research Projects Agency (DARPA) during early 1980s mainly for military applications. The purpose of the project was to place the radars and antennas far away from the command and control centers due to the development of new radar-seeking missiles at that time. However, the technology has initially limited applications mainly due to high manufacturing cost of wide bandwidth lasers. Later, mass production of lasers and photodetectors and the need for communication networks covering wide areas eventually lead to RoF projects like Quasi-Sync in Europe and Simulcast in US. Since then RoF technology has moved forward a long way. Now, it is thought as one of the most potential technologies for 4-G communication. Major players in telecommunication industry like Nortel and Telus have already implemented RoF links for commercial purposes. It is expected RoF technology will be widely used for home networking applications over the coming years.

A basic RoF configuration consists of a two-way interface that contains a laser transmitter and photodiode receiver which connects the base station transmitters and receivers to a pair of single mode optical fibers [16] as shown in Fig. 2.1. At the other end of the fibers is a remote unit that uses a similar photodiode receiver and laser transmitter to convert optical signals to and from an antenna. 


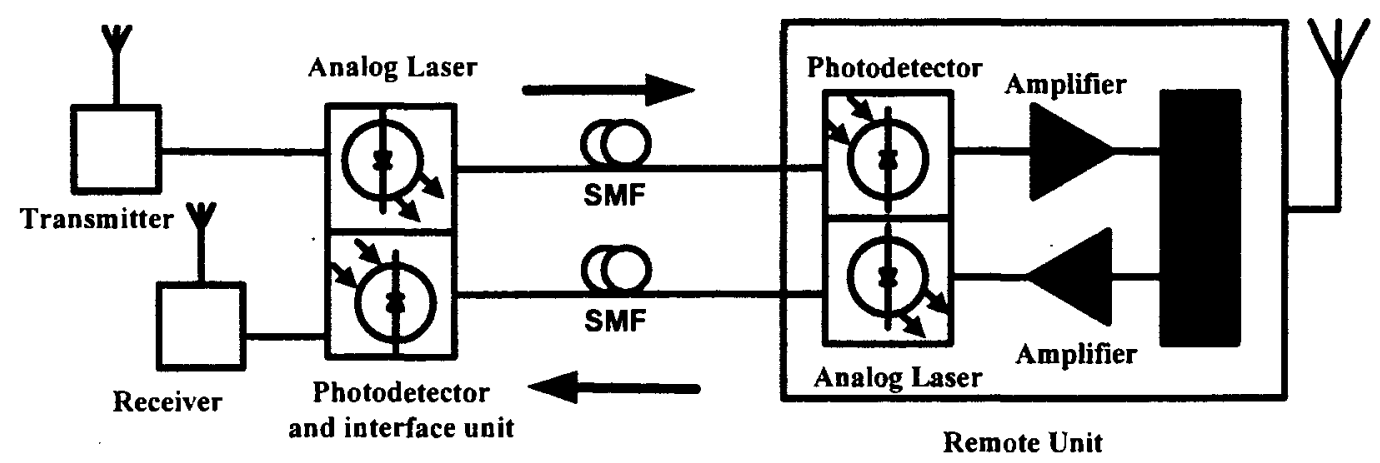

Figure 2.1 Basic RoF system [16].

\subsection{Advantages of RoF Systems}

Some of the benefits of RoF technology are discussed below-

- Low attenuation - The transmission loss optical fiber is very low. Table 2.1 shows a relative comparison of transmission loss and cost using standard single mode fiber (SMF), standard coaxial cable and special coaxial cable for high frequency application. The table shows that SMF will perform much better than coaxial cable for transmission of high frequency signal.

Table 2.1 Comparison between SMF and coaxial cable.

\begin{tabular}{|c|c|c|c|}
\hline Frequency & SMF & $\begin{array}{c}\text { Standard coaxial } \\
\text { cable }\end{array}$ & $\begin{array}{c}\text { Special coaxial } \\
\text { cable }\end{array}$ \\
\hline $1 \mathrm{GHz}$ & $0.2 \mathrm{~dB} / \mathrm{km}$ & $0.29 \mathrm{~dB} / \mathrm{Ft}$. & $0.2 \mathrm{~dB} / \mathrm{Ft}$. \\
\hline
\end{tabular}




\begin{tabular}{|c|c|c|c|}
\hline $18 \mathrm{GHz}$ & $0.2 \mathrm{~dB} / \mathrm{km}$ & $4 \mathrm{~dB} / \mathrm{Ft}$. & $1.1 \mathrm{~dB} / \mathrm{Ft}$. \\
\hline Cost & $20 \mathrm{USD} / \mathrm{km}$ & $50 \mathrm{USD} / \mathrm{Ft}$. & $70 \mathrm{USD} / \mathrm{Ft}$. \\
& & & \\
\hline
\end{tabular}

- Wide bandwidth - One of the most important advantages of optical fiber communication is that it has very large bandwidth. As a result it is transparent to any type of network or application.

- Immune of interference - In optical fiber communication information is transmitted by modulating the light with $\mathrm{RF}$ signal. As a result RoF communication is free from electromagnetic interference. Also, RoF does not suffer from multipath interference which is a common problem in normal wireless communication.

- Low RF power - Low RF power antennas are required if fiber is used for distribution. This has many advantages. Reducing the RF power radiated by antennas reduce interference. Reducing emitted power is also environmentally friendly.

- Economical solution - Optical fiber is very cheap (Table 2.1). If low cost lasers and photodetectors are used RoF can provide a very economic solution for 
broadband access networks. Also optical fiber communication is more reliable and costs less for maintenance.

- Easier cell planning - In conventional wireless communication cell planning is a complicated task. Designers have to be careful that interference from the edge one cell does not affect the other. Also RoF reduces the number of handovers.

\subsection{Applications of RoF Systems}

Some of the applications of RoF technology are discussed below-

- CATV distribution systems - RoF technology is widely used to provide video distribution services in $40 \mathrm{GHz}$ band over the last decade. Fiber to home networks (FTTH) are used for multi point video distribution service (MVDS) and transmission of IEEE 802.16 broadband services.

- Local access networks - Recently there is new grown interest in transmitting wireless signals over fiber networks for local access network (LAN) applications. Fiber can provide a low cost communication under office environments. Over the last few years many work has been done on transmission of $2.4 \mathrm{GHz}$ IEEE 802.1lb over fiber [17-18] and it is found fiber has superb potential for this application. In December 2008 Nortel has inaugurated their solution for Ethernet over fiber in USA for LAN applications. 
- Cellular networks - Under densely populated areas RoF fiber can become very handy for transmission of mobile signals. In fact during 2000 Sydney Olympics Allen Telecom installed BriteCell, a fiber optic-based mobile communications system. According to press releases, on the opening day of the Olympics, over 500,000 wireless calls were made from Olympic Park venues. RoF systems are an effective solution under situations like this where the number subscriber increase suddenly in an area at particular time. In Canada Telus has established their network for transmission of mobile signals through optical fiber.

- Vehicular Technology - RoF is also used for toll collection data transfer, intelligent transport and road to vehicle communication systems. Also a wireless sensor network with fiber distribution is used for traffic control and traffic data collection. 


\section{Chapter 3 UWB Overview}

UWB is not a new technology. The first spark-gap radios developed in early nineteenth century were in fact UWB radios. In early days the advantages of UWB cannot be fully exploited because of immature technology. However, over the course of the last half a century, the UWB signals have re-emerged. In this chapter the early history of UWB, legislation and its relentless march towards current position is presented.

\subsection{Historical Perspective}

James Clerk Maxwell formulated the concept of electricity and magnetism in 1864. His theory predicted that energy can be transported through materials and through space at a finite velocity by the action of electric and magnetic waves moving through time and space [19-20]. In 1886 Rudolf Hertz, created and detected such oscillations over a distance of several meters in his lab using a spark-gap apparatus to generate radio energy. Through these experiments, the era of wireless begun. Later, Alexander S. Popov and Nikola Tesla developed their tuned resonant transmitter and receiver circuits. In 1895 A. Popov transmitted and received signals across a distance of $600 \mathrm{~m}$. In that same year, Guglielmo Marconi, using a Hertz oscillator, antenna, and receiver very similar to Popov's, successfully transmitted and received signals within the limits of his father's estate at Bologna, Italy. In 1897 Marconi, formed his first company, Wireless Telegraph and Signal Company, in Britain, and began manufacturing wireless sets in 1898 . By 
1901, Marconi bridged the $3,000-\mathrm{km}$ distance between St. John's Newfoundland and Cornwall, on the southwest tip of England, using Morse code transmissions. With this achievement, Marconi introduced long-distance communication. Marconi brought his technology to the United States in 1899 with the Marconi Company. Soon, he controlled patents for the tuner, patented by British inventor Oliver J. Lodge in 1898 and for the John A. Fleming valve (vacuum tube) of 1904 that acted as a diode tube to efficiently detect wireless signals. The Lodge patent is particularly interesting in that it offers advantages in transmitting and receiving tuning circuits so that multiple stations may operate side by side in the radio spectrum without mutual interference. The movement was primarily away from wideband signals because at that time there was no way to effectively recover the wideband energy emitted by a spark-gap transmitter. There was also no way to discriminate among many such wideband signals in a receiver. Wideband signals simply caused too much interference with one another to be useful.

\subsection{UWB Standardization}

During February 2002, the FCC of United States approved a spectrum in the range of 3.1-10.6 GHz to be available for the unlicensed use of UWB signals. The US FCC provided the following guidelines-

\section{Definition of UWB}

According to FCC UWB signal either occupies at least $500 \mathrm{MHz}$ of spectrum or its $10 \mathrm{~dB}$ bandwidth is at least $20 \%$ of the size of the center frequency. 


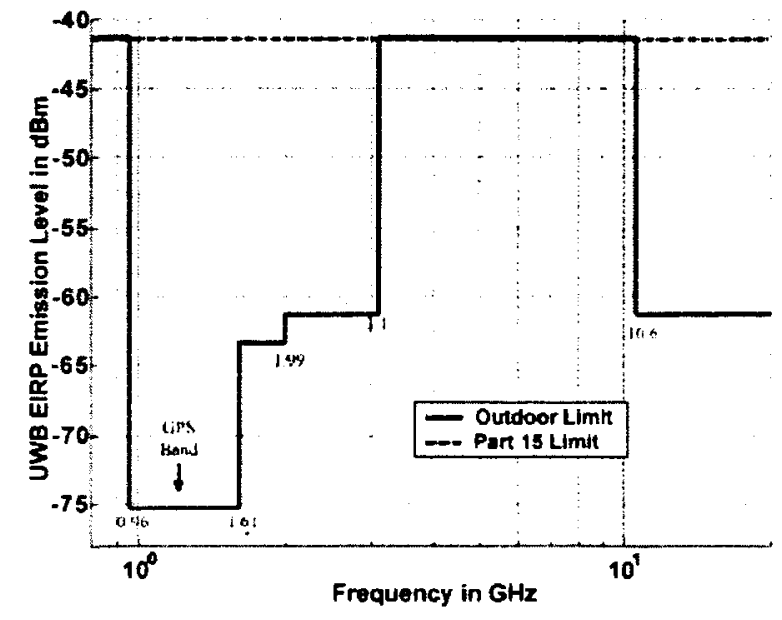

Figure 3.1 FCC UWB emission spectrum [1].

\section{Spectrum Allocation}

FCC requires that the spectrum must use the band allocation showed in Fig. 3.1.

\section{Emission Limits}

The power spectral mask is depicted in Fig. 3.1. The average in band power emission is limited to $-41.3 \mathrm{dBm} / \mathrm{MHz}$ in terms of effective isotropic radiated power (EIRP).

\subsubsection{IEEE}

IEEE is one of the pioneers in standardizing the UWB. In December 2002, IEEE 802.15.3a was formed to address high data rate UWB. The task groups eventually choose two technologies for UWB: MB-OFDM or Direct sequence (DS) CDMA. MB OFDM 
approach has more supporters but it failed to achieve super majority (70\%). The battle between two sides became more political and the draft standard came to a standstill.

\subsubsection{WiMedia}

The supporters for MB OFDM approach bypassed IEEE and started their own standardization effort. The group was led by Intel, Texas Instruments, Nokia, HP, Samsung, and Sony and was followed by many others. They formed WiMedia Alliance which is a nonprofit organization that promotes MB OFDM UWB.

\subsubsection{ECMA}

ECMA International is a standardization body which aims to facilitate the standardization of technologies in information and communication technology. WiMedia submitted its specification to ECMA in 2005. In the same year ECMA approved the publication of the first combined PHY layer and MAC sub layer specification in single document called ECMA-368. ECMA-368 has been widely accepted by most UWB PHY developers. In December 2007 the ECMA-368 underwent another revision. ECMA-368 has also been published by International Organization for Standardization (ISO) and European Telecommunication Standards Institute (ETSI) named ISO/IEC 26907 and ETSI IS 102455 respectively.

\subsubsection{Current Worldwide Regulations}

US FCC is the pioneer in standardizing the UWB. Other countries quickly followed FCC initiative. The current worldwide regulation for UWB is presented in Fig. 3.2. After FCC Japan's Ministry of Internal affairs and Communications (MIC) made its 
rulings in September 2005. MIC introduced some new concepts in their rulings. One of them is the introduction of Detection-And-Avoidance (DAA) scheme. In DAA scheme UWB transceiver first send a beacon signal to detect any other narrowband transmitters in its close proximity and if an interferer is present UWB devices switch to next available frequency sub-band. On February 21, 2007 European Commission (EC) released a policy document in favour of allocating spectrum for UWB. The policy requires a DAA scheme for Band Group 1. Ministry of Information and Communications (MIC) in Korea adopted a UWB spectrum similar to Japan and EC. Chinese Ministry of information and Industry (MII) has not yet finalized its policy on UWB. MII's draft resolution requires DAA scheme for sub-band 3 of Band group 1.

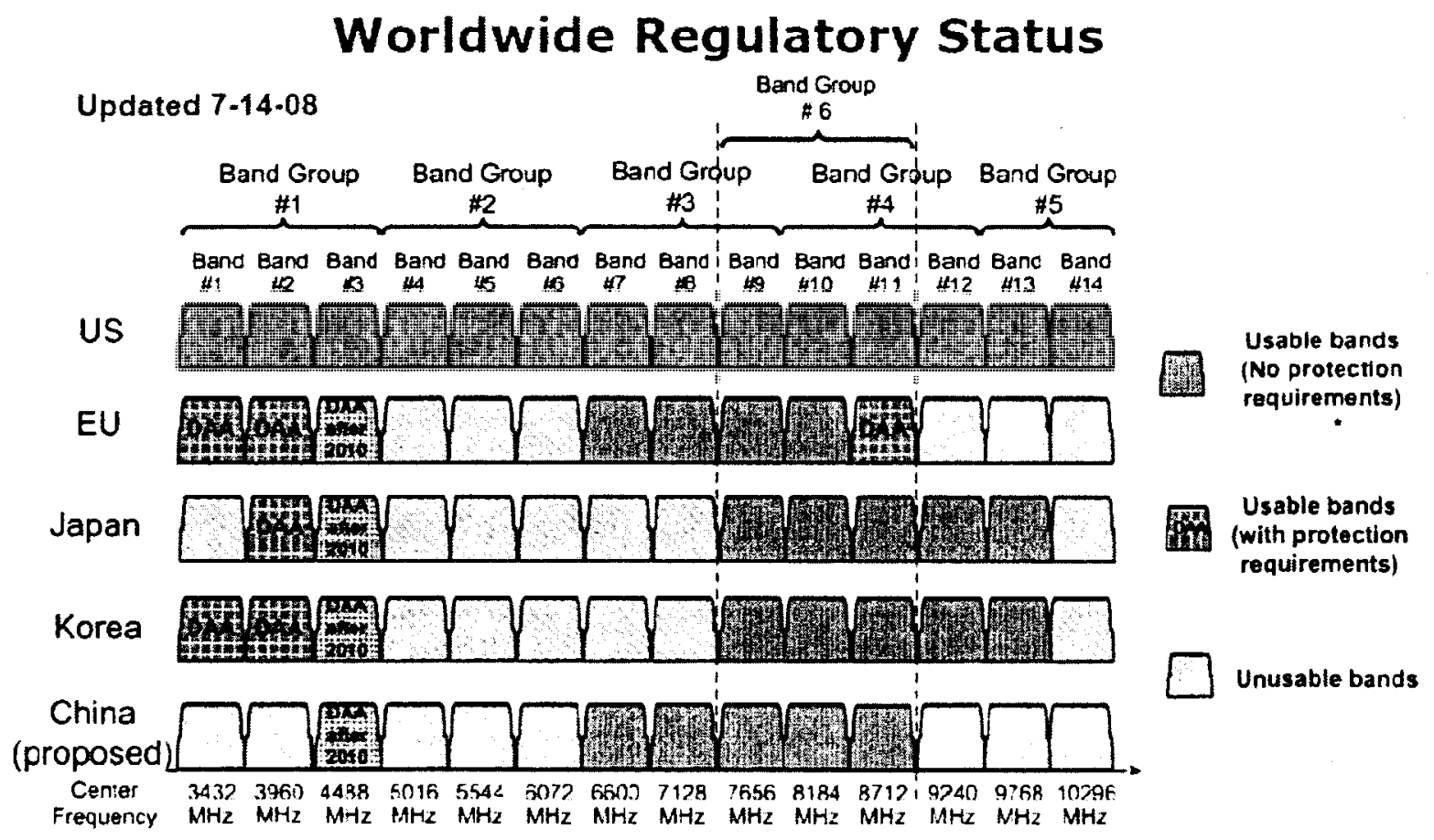

Figure 3.2 Current worldwide regulation for UWB [21]. 


\subsection{MB OFDM UWB}

FCC only provides a guideline for UWB rather than giving details about the PHY layer implementation. So, several UWB transmission techniques have been proposed. These techniques can be categorized in two major groups: single band and multi-band UWB. The single band approach is implemented by direct modulation of information into a sequence of impulse like waveforms which occupy the available bandwidth of $7.5 \mathrm{GHz}$ [22]. Multiple users are supported by using a complex time hopping sequence. But building devices to handle ultra short pulse signal is quite challenging. Also this approach requires a very complex receiver structure. In MB approach the information is processed over a small bandwidth of at least $500 \mathrm{MHz}$ [2]. This reduces and design complexity and provides flexible worldwide compliance.

An MB OFDM signal consists of 128 subcarriers using quadrature phase shift keying (QPSK) for lower bit rates. However, advanced dual carrier modulation technique is used for bit rate of higher than $200 \mathrm{Mb} / \mathrm{s}$. Figure 3.4(a) shows RF spectrum for the first three-bands of the 14 bands with bit rate of $200 \mathrm{Mb} / \mathrm{s}$ for each band, and the three bands are centered at frequency of $f_{l}=3.432, f_{2}=3.96$ and $f_{3}=4.488 \mathrm{GHz}$. The signal follows simple frequency hoping sequences like $f_{l}, f_{2}$ and $f_{3}$ as shown in Fig. 3.3(b) that is the spectrogram. Figure 3.3(c) shows the three bands in the time domain.

In the next sections the physical layer of MB OFDM system is described as proposed in the IEEE 802.15.3a and ECMA-368 standard. 


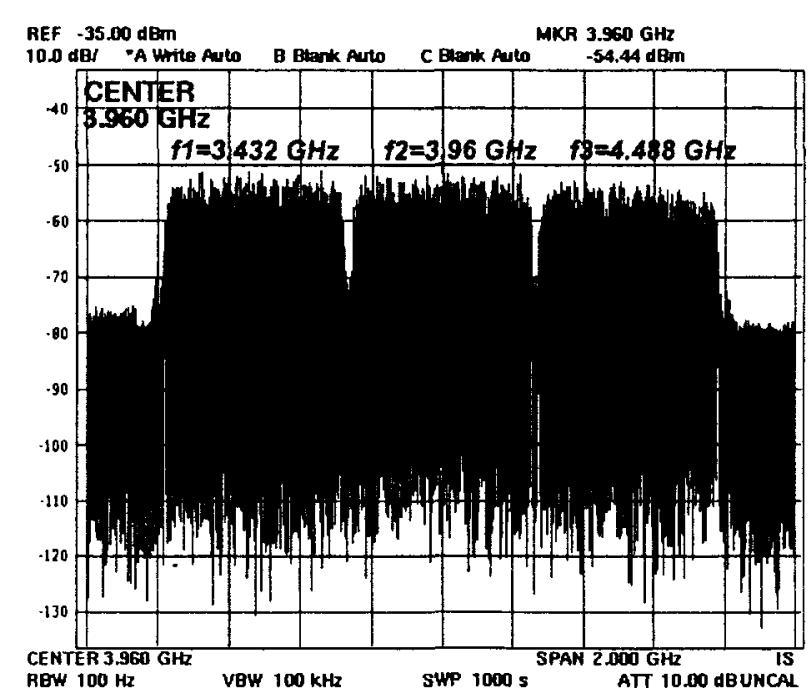

(a)

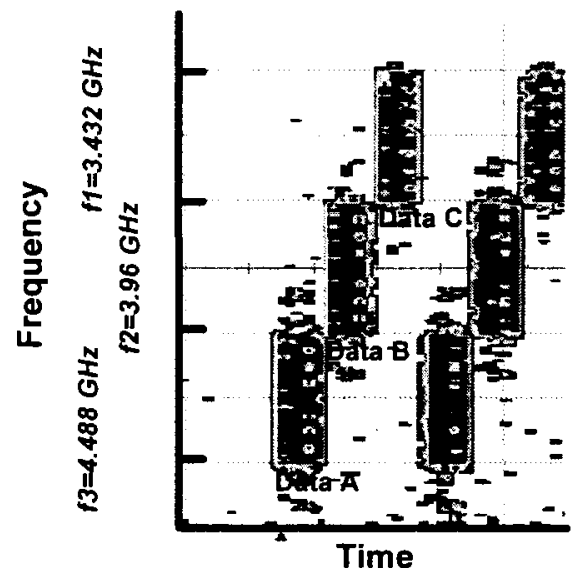

(b)

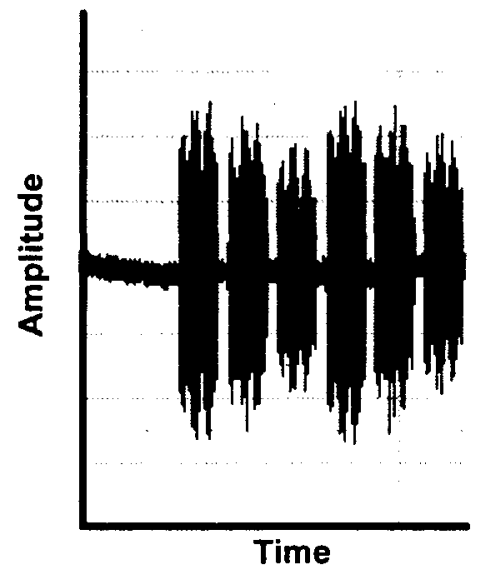

(c)

Figure 3.3 First three channels of MB-OFDM UWB wireless in (a) frequency domain, (b) frequency-time domain, and (c) time domain.

\subsubsection{MB OFDM UWB Wireless Transmitter and Receiver}

The structure of an MB OFDM transmitter and receiver is shown in Fig. 3.4. At the transmitter the input data stream is first scrambled. The purpose of the data scrambler is to convert a data bit sequence into pseudorandom sequence that is free from long 
strings of simple patterns such as ones and zeroes. Then the data is conventionally encoded which improves the signal noise ratio (SNR) due to addition of patterns of redundancy. The basic coding rate is usually called mother encoding rate. To obtain other coding rates the coded sequence again punctured. Puncturing is a procedure for omitting some encoded bits at the transmitter and inserting dummy zero into the sequence received at the receiver in place of the bits omitted. The third block in the UWB transmitter is the bit interleaver. Bit interlaever provides robustness against burst errors, which consists of a symbol interleaving followed by tone interleaving. Then the bit interleaved sequence is mapped into a sequence of QPSK symbols according to gray coded constellation as shown in Fig. 3.5 and Table 3.2.

The complex valued sequence from constellation mapper is then OFDM modulated. The sequence is first converted into parallel sequence. After adding pilot, guard and null tones the inverse fast Fourier transform (IFFT) is performed. The OFDM signal is up-converted to a specific sub band. Each OFDM symbol contains 128 subcarriers with symbol duration of $T_{S}=242.42 \mathrm{~ns}$ as presented in Table 3.2. After digital to analog conversion, each band or channel will be imposed on a RF carrier as specified in Table 3.3 and Fig. 3.6. 

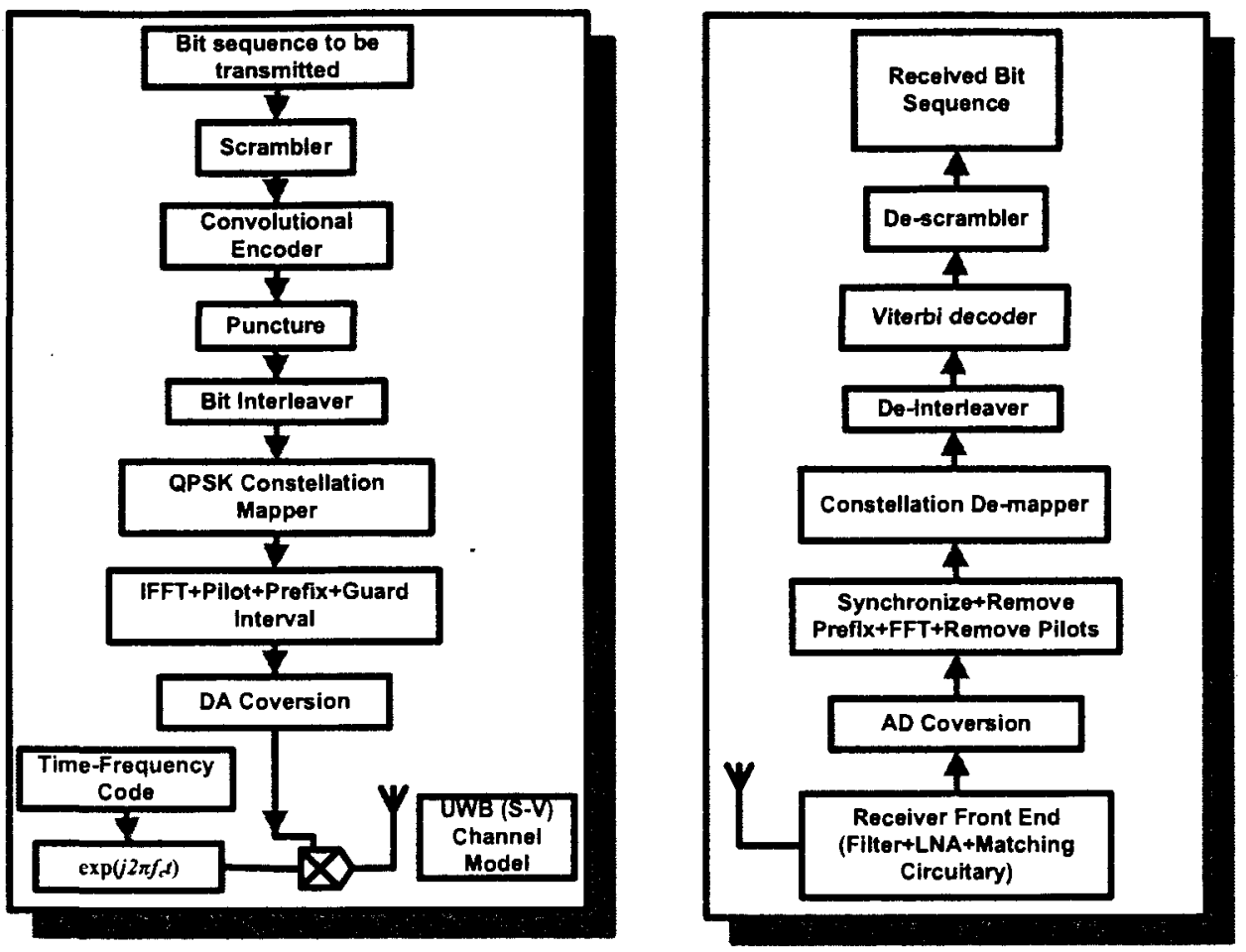

Figure 3.4 UWB transmitter and receiver [2].

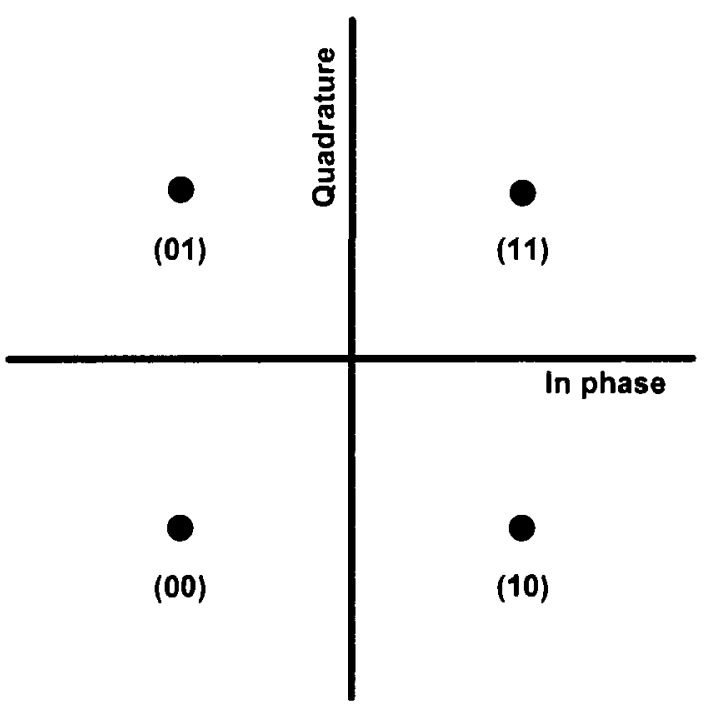

Figure 3.5 QPSK constellation [2]. 
Table 3.1 - QPSK Encoding Table [2].

\begin{tabular}{|l|l|l|}
\hline Input Bit & I-out & Q-out \\
\hline 00 & -1 & -1 \\
\hline 01. & -1 & 1 \\
\hline 10 & 1 & -1 \\
\hline
\end{tabular}

The operation of the receiver is similar to the transmitter. The high frequency signal is passed through a preselect filter, amplified with an RF amplifier and down converted into baseband. After performing synchronization fast Fourier transform (FFT) is done to demodulate QPSK symbols from the received OFDM signal. From the QPSK symbols an estimated bit sequence is reconstructed and de-interleaved. The QPSK sequence is then channel decoded using Viterbi algorithm [2]. Finally the decoder output is descrambled to desired bit sequence.

\subsubsection{Timing and Synchronization Parameters}

The main timing related parameters for an MB OFDM system are shown in Table 3.2 . 
Table 3.2 Timing Related Parameters of MB OFDM [2].

\begin{tabular}{|c|c|c|}
\hline Parameter & Description & Value \\
\hline$f_{s}$ & Sampling frequency & $528 \mathrm{MHz}$ \\
\hline $\mathrm{N}_{\mathrm{FFT}}$ & Total number of subcarriers (FFT & 128 \\
\hline $\mathrm{N}_{\mathrm{D}}$ & Number of data subcarriers & 100 \\
\hline $\mathrm{N}_{\mathrm{P}}$ & Number of pilot subcarriers & 12 \\
\hline $\mathrm{N}_{\mathrm{G}}$ & Number of guard subcarriers & 10 \\
\hline $\mathrm{N}_{\mathrm{T}}$ & Total number of subcarriers used & $122\left(=N_{D}+N_{P}+N_{G}\right)$ \\
\hline$\overline{\Delta f}$ & Subcarrier frequency spacing & $4.125 \mathrm{MHz}\left(=\mathrm{f}_{\mathrm{s}} / \mathrm{N}_{\mathrm{FFT}}\right)$ \\
\hline$\overline{T_{F F T}}$ & IFFT and FFT period & $242.42 \mathrm{~ns}(=1 / \Delta \mathrm{f})$ \\
\hline $\mathrm{T}_{\mathrm{CP}}$ & Cyclic prefix duration & $60.61 \mathrm{~ns}(=32 / 528 \mathrm{MHz})$ \\
\hline $\mathrm{T}_{\mathrm{Gl}}$ & Guard interval duration & $9.47 \mathrm{~ns}(=5 / 528 \mathrm{MHz})$ \\
\hline $\mathrm{T}_{\text {SYM }}$ & Symbol duration & $\begin{array}{c}312.5 \mathrm{~ns}\left(=\mathrm{T}_{\mathrm{FFT}}+\mathrm{T}_{\mathrm{CP}}+\right. \\
\left.\mathrm{T}_{\mathrm{GI}}\right)\end{array}$ \\
\hline
\end{tabular}




\subsubsection{Modes of Operation}

Table 3.3 and Fig. 3.6 show different bands MB OFDM UWB. Group 1 is mandatory and other groups are optional. This is mainly due to the unavailability of the CMOS hardware at higher frequencies. As stated earlier the bands are hoped using simple sequences as shown in Table 3.4 to avoid unwanted interference and support multiple users.

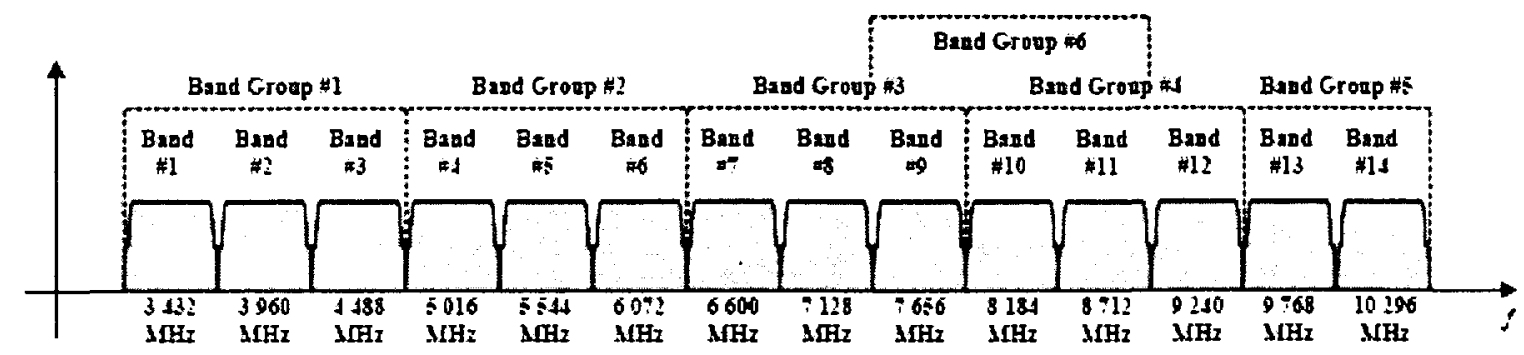

Figure 3.6 Band Group allocation for MB OFDM UWB [4].

Table 3.3: Band Group allocation for MB OFDM UWB [4].

\begin{tabular}{|c|c|c|c|c|}
\hline Band & BAND & $\begin{array}{c}\text { Lower Frequency } \\
(\mathrm{MHz})\end{array}$ & $\begin{array}{c}\text { Center Frequency } \\
(\mathrm{MHz})\end{array}$ & $\begin{array}{c}\text { Upper Frequency } \\
(\mathrm{MHz})\end{array}$ \\
\hline 1 & 1 & 3168 & 3432 & 3696 \\
\cline { 2 - 5 } & 2 & 3696 & 3960 & 4224 \\
\hline
\end{tabular}




\begin{tabular}{|c|c|c|c|c|}
\hline & 3 & 4224 & 4488 & 4752 \\
\hline \multirow[t]{3}{*}{2} & $\overline{4}$ & 4752 & 5016 & 5280 \\
\hline & 5 & 5280 & 5544 & 5808 \\
\hline & 6 & 5808 & 6072 & 6336 \\
\hline \multirow[t]{3}{*}{3} & 7 & 6336 & 6600 & 6864 \\
\hline & 8 & 6864 & 7128 & 7392 \\
\hline & 9 & 7392 & 7656 & 7920 \\
\hline \multirow[t]{3}{*}{4} & 10 & 7920 & 8184 & 8448 \\
\hline & 11 & 8448 & 8712 & 8976 \\
\hline & 12 & 8976 & 9240 & 9504 \\
\hline \multirow[t]{2}{*}{5} & 13 & 9504 & 9768 & 10032 \\
\hline & 14 & 10032 & 10296 & 10560 \\
\hline \multirow[t]{3}{*}{6} & 9 & 7392 & 7656 & 7920 \\
\hline & 10 & 7920 & 8184 & 8448 \\
\hline & 11 & 8448 & 8712 & 8976 \\
\hline
\end{tabular}


Table 3.4: Time frequency codes for Group-1 MB OFDM system [4].

\begin{tabular}{|c|c|c|c|c|c|c|}
\hline TFC & \multicolumn{6}{|c|}{ Hoping Sequence } \\
\hline 1 & 1 & 2 & 3 & 1 & 2 & 3 \\
\hline 2 & 1 & 3 & 2 & 1 & 3 & 2 \\
\hline 3 & 1 & 1 & 2 & 2 & 3 & 3 \\
\hline 4 & 1 & 1 & 3 & 3 & 2 & 2 \\
\hline 5 & 1 & 1 & 1 & 1 & 1 & 1 \\
\hline 6 & 2 & 2 & 2 & 2 & 2 & 2 \\
\hline 7 & 3 & 3 & 3 & 3 & 3 & 3 \\
\hline 8 & 1 & 2 & 1 & 2 & 1 & 2 \\
\hline 9 & 1 & 3 & 1 & 3 & 1 & 3 \\
\hline 10 & 2 & 3 & 2 & 3 & 2 & 3 \\
\hline
\end{tabular}




\subsubsection{Data Rate Dependent Parameters}

Table 3.5 and Table 3.6 lists data rate dependent parameters and receiver sensitivity for an MB OFDM UWB system, respectively. Maximum permissible relative constellation error or root mean square error vector magnitude (EVM) is depicted in

Table 3.7.

Table 3.5: Data rate dependent parameters MB OFDM system [4].

\begin{tabular}{|c|c|c|c|c|c|c|}
\hline $\begin{array}{c}\text { Data } \\
\text { Rate } \\
(\mathrm{Mb} / \mathrm{s})\end{array}$ & Modulation & $\begin{array}{c}\text { Rate } \\
(\mathrm{R})\end{array}$ & $\begin{array}{c}\text { Frequency } \\
\text { Spreading }\end{array}$ & $\begin{array}{c}\text { Time } \\
\text { Spreading }\end{array}$ & $\begin{array}{c}\text { Coded Bits } \\
\text { /6 OFDM } \\
\text { Symbol }\end{array}$ & $\begin{array}{c}\text { Info Bits / } \\
6 \text { OFDM } \\
\text { Symbol }\end{array}$ \\
\hline 53.3 & QPSK & $1 / 3$ & YES & YES & 300 & 100 \\
\hline 80 & QPSK & $1 / 2$ & YES & YES & 300 & 150 \\
\hline 106.7 & QPSK & $1 / 3$ & NO & YES & 600 & 200 \\
\hline 160 & QPSK & $1 / 2$ & NO & YES & 600 & 300 \\
\hline 200 & QPSK & $5 / 8$ & NO & YES & 600 & 375 \\
\hline 320 & DCM & $1 / 2$ & NO & NO & 1200 & 600 \\
\hline 400 & DCM & $5 / 8$ & NO & NO & 1200 & 750 \\
\hline 480 & DCM & $3 / 4$ & NO & NO & 1200 & 900 \\
\hline
\end{tabular}


Table 3.6: Sensitivity of UWB receiver [4].

\begin{tabular}{|l|c|}
\hline Data Rate $(\mathrm{Mb} / \mathrm{s})$ & Minimum Receiver Sensitivity $(\mathrm{dBm})$ \\
\hline 53.3 & -80.8 \\
\hline 80 & -78.9 \\
\hline 106.7 & -77.8 \\
\hline 160 & -75.9 \\
\hline 200 & -74.5 \\
\hline 320 & -72.8 \\
\hline 400 & -71.5 \\
\hline 480 & -70.4 \\
\hline
\end{tabular}

Table 3.7: Permissible Relative Constellation Error (EVM).

\begin{tabular}{|l|c|c|c|}
\hline & \multicolumn{3}{|c|}{ Relative Constellation RMS Error } \\
\hline & No TX & TX Attenuation & \\
Data Rate & Attenuation & TX Attenuation of 8, \\
& & (All TFCs) & $10,12 \mathrm{~dB}$ (All TFCs) \\
\hline $53.3 \mathrm{Mb} / \mathrm{s}, 80 \mathrm{Mb} / \mathrm{s}$, & $-17.0 \mathrm{~dB}$ & $-15.5 \mathrm{~dB}$ & $-14.5 \mathrm{~dB}$ \\
$106.7 \mathrm{Mb} / \mathrm{s}, \quad 160$ & & & \\
$\mathrm{Mb} / \mathrm{s}, 200 \mathrm{Mb} / \mathrm{s}$ & & & \\
\hline $320 \mathrm{Mb} / \mathrm{s}, 400$ & $-19.5 \mathrm{~dB}$ & $-18.0 \mathrm{~dB}$ & $-17.0 \mathrm{~dB}$ \\
$\mathrm{Mb} / \mathrm{s}, 480 \mathrm{Mb} / \mathrm{s}$ & & & \\
\hline
\end{tabular}




\subsection{Mathematical Framework for MB OFDM UWB}

The MB OFDM RF signal $\left(y_{R F}(\mathrm{t})\right)$ is related to the complex baseband signal $\left(x_{k}(\mathrm{t})\right)$ of the $k^{t h}$ OFDM symbol as [2]

$$
y_{R F}(t)=\operatorname{Re}\left\{\sum_{k=0}^{N-1} x_{k}\left(t-k T_{S Y M}\right) \exp \left(j 2 \pi f_{c} t\right)\right\}
$$

where $\operatorname{Re}(\cdot)$ represents the real part of a complex variable, $x_{k}(t)$ is the complex baseband signal of the $k^{\text {th }}$ OFDM symbol and is nonzero over the interval from 0 to $T_{S Y M}, N$ is the number of OFDM symbols, $T_{S Y M}$ is the symbol interval, and $f_{c}$ is the center frequency for the $\mathrm{k}^{\text {th }}$ band.

The OFDM symbols $x_{k}(\mathrm{t})$ can be constructed using inverse Fast Fourier transform (IFFT) with a certain coefficients $C_{n}$, which can consist of data symbols, pilots, and training symbols,

$$
\begin{aligned}
& x_{k}(t)=\sum_{n=-N_{S T} / 2}^{N_{S T} / 2} C_{n} \exp \left(j 2 \pi n \Delta f\left(t-T_{C P}\right)\right) \\
& t \in\left[T_{C P}, T_{F F T}+T_{C P}\right], \text { otherwise } 0
\end{aligned}
$$

where, $N_{S T}=128$ is the total number of subcarrier used, $\Delta f=B W / N=4.125 \mathrm{MHz}$ is the subcarrier frequency spacing, $n$ is the subcarrier number, $T_{C P}=60.61 \mathrm{~ns}$ is the cyclic prefix, $T_{F F T}=1 / \Delta f=242.42 n s$ is the IFFT/FFT period, and $B W$ is the signal bandwidth. 


\subsection{UWB over Fiber Technologies}

FCC imposed a strict spectral mask for the UWB to avoid interference with existing narrow band wireless systems. It is required that allowed effective isotropic radiated power (EIRP) is $-41.3 \mathrm{dBm} / \mathrm{MHz}$, and the total transmitted power from a UWB antenna is limited to $0.5 \mathrm{~mW}$ only. In order to satisfy the EIRP requirement defined by FCC, UWB signals are designed to have noise-like properties and the energy of the high frequency signal is spread over a very large bandwidth. This is why distribution of UWB over coaxial cable is exceedingly expensive. Optical fiber provides an excellent alternative for distribution of UWB signals due to its low loss, low cost and wide bandwidth characteristics.

First the UWB signal is generated from UWB transmitter as depicted in 3.3.1. This signal directly drives a laser or an optical modulator, and then MB OFDM UWB signal becomes optical subcarriers. After fiber distribution photodiode directly converts optical UWB subcarrier to UWB radio signal, which will be emitted by a UWB antenna at user's location. All the features of UWB over fiber will be discussed in details in the upcoming chapters. 


\section{Chapter 4 Experimental Setups and Theoretical}

\section{analysis}

In this chapter we investigate the experimental setup for UWB over fiber transmission and perform theoretical analysis.

\subsection{Experimental System Configuration for UWB over Fiber}

\section{System}

In this section we will discuss experimental setup for single channel and WDM UWB over fiber systems.

\subsubsection{Single Channel UWB over Fiber System}

The considered system setup for the performance evaluation of UWB over fiber is shown in Fig. 4.1 and 4.2. A commercially available evaluation board, DV9110M, from WisAir, providing MB-OFDM compliant modulation with three WiMedia sub-bands allocated at center frequency of $3.432,3.96$ and $4.488 \mathrm{GHz}$, is used for MB OFDM UWB generation and reception. Number of transmitted packets is $10^{6}$. Each packet has 1024 octets. The RF signal is amplified and variable attenuator (VA) is used to vary the RF power. A continuous wave (CW) light from tunable laser source (Anritsu MG9541A) has a wavelength of $1550 \mathrm{~nm}$, linewidth of $800 \mathrm{KHz}$, intrinsic relative intensity noise of -155 $\mathrm{dB} / \mathrm{Hz}$ and output optical power of $0 \mathrm{dBm}$. For experiments concerning $\mathrm{RNN}$ a continuous wave $(\mathrm{CW})$ light from multi-channel fiber optic source module ILX Lightwave FOM$7900 \mathrm{~B}$ is set to have a wavelength of $1553.3 \mathrm{~nm}$ and output power from -6 to $5 \mathrm{dBm}$. The 
linewidth of the laser can be tuned to either $30 \mathrm{MHz}$ or $1 \mathrm{GHz}$. The $\mathrm{CW}$ light is injected into a $10.7 \mathrm{~Gb} / \mathrm{s}$ dual-electrode Mach-Zehnder modulator (DE-MZM) from Fujitsu, driven by the UWB signal from the output of the DV9110M Tx module. The MZM has 6 $\mathrm{dB}$ insertion loss and an extinction ratio of $28.5 \mathrm{~dB}$.

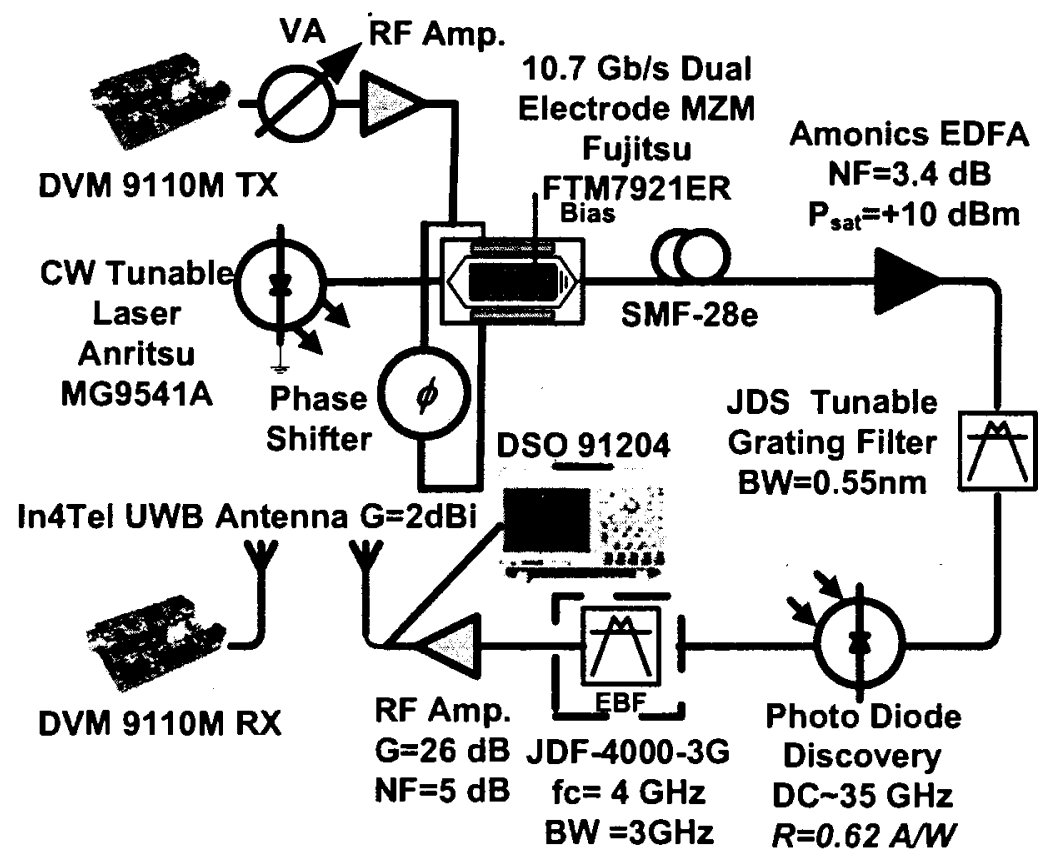

Figure 4.1 Experimental setup for externally modulated MB-OFDM UWB over fiber system.

To obtain optical single side band (OSSB) modulation the UWB signal is applied to both branches of the DE-MZM through a hybrid coupler and bias-T with a $90^{\circ}$ phase shifter in one branch. On the contrary, to generate optical double side band (ODSB), the signal is directly applied to both electrodes. The modulated lightwave is sent through $\mathrm{SMF}$, with fiber loss of $\alpha=0.21 \mathrm{~dB} / \mathrm{km}$ and chromatic dispersion of $17 \mathrm{ps} /(\mathrm{nm} . \mathrm{km})$. We consider UWB over fiber with back-to-back, 20 and $40 \mathrm{~km}$ of fiber transmission. After 
fiber transmission, the UWB signal is optically amplified by an erbium doped fiber amplifier (EDFA) and then a JDS tunable grating filter with bandwidth of $0.55 \mathrm{~nm}$ and insertion loss of $2 \mathrm{~dB}$ is used before being detected by a high speed photodiode (Discovery DSC-740 with 3-dB bandwidth of $35 \mathrm{GHz}$ and responsivity of $0.62 \mathrm{~A} / \mathrm{W}$ ).

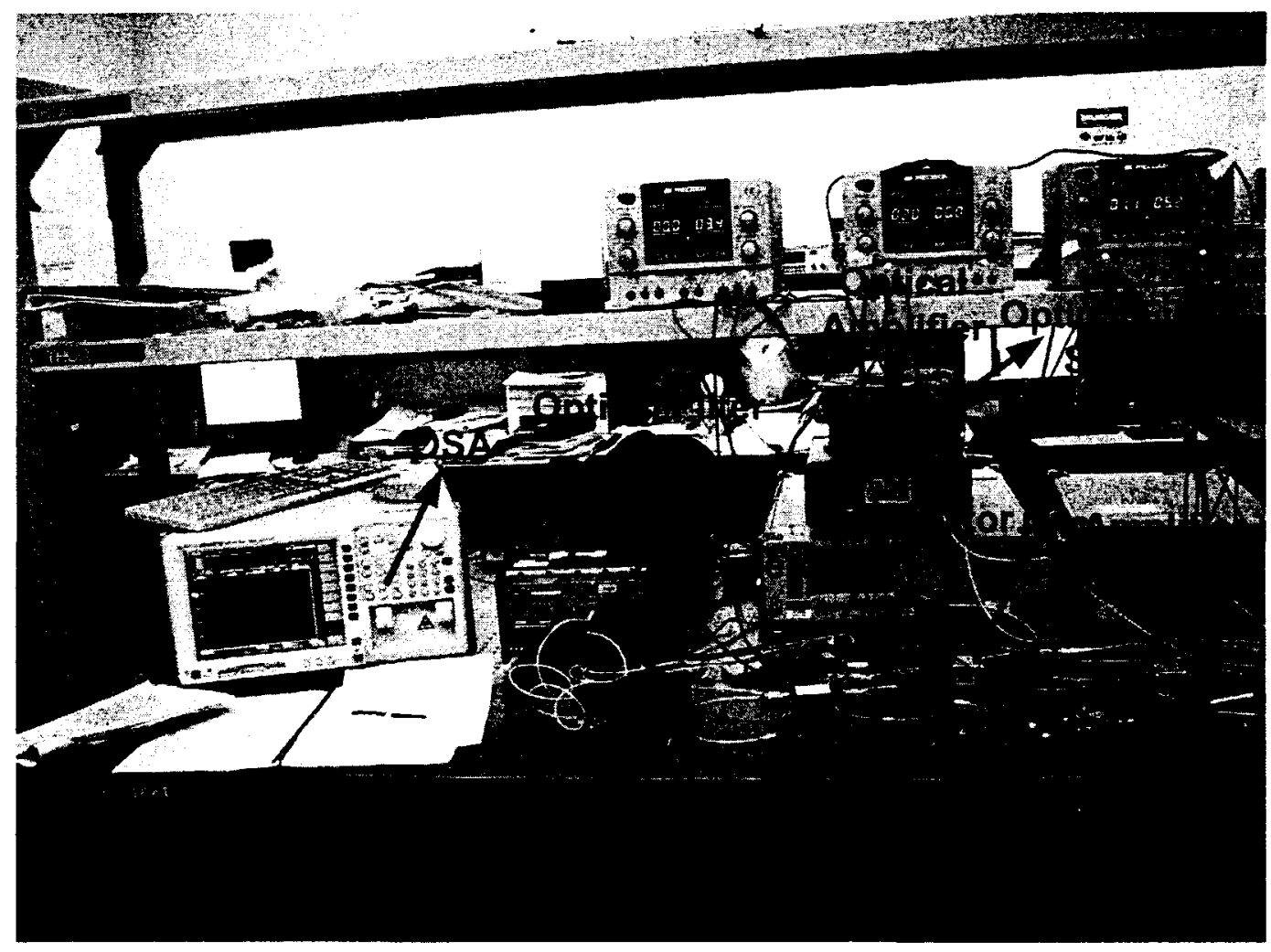

Figure 4.2 Photograph of experimental setup for externally modulated MB-OFDM UWB over fiber system. 
The EDFA gain is controlled in such a way that the insertion loss of all optical components and fiber is compensated. After photodetection the UWB signal is electrically filtered by a JDF electrical bandpass filter (EBF) of bandwidth of $3 \mathrm{GHz}$ and then amplified by a broadband RF amplifier from SHF (SHF-810) $(>30 \mathrm{GHz})$ as shown in Fig. 4.1. The broadband photodetector combined with the broadband RF amplifier is referred to "ideal" optical receiver. Here the "ideal" optical receiver means that it does not introduce amplitude and phase distortion to the OFDM signal. The received signal is evaluated with a high speed real time oscilloscope DSO 91204 from Agilent Technologies. The received UWB signal is launched to air by an antenna. Then the UWB wireless signal is received and processed by DV9110M Rx module after $1 \mathrm{~m}$ wireless over air transmission.

\subsubsection{MB-OFDM UWB over Fiber System and In-band Interferers}

The considered system setup for the performance evaluation of UWB over fiber under the presence of in-band interferers is shown in Fig. 4.3. An arbitrary waveform generator, AWG 7122B, from Tektronix with $12 \mathrm{GHz}$ RF bandwidth is used for UWB signal generation. First the MB-OFDM UWB signals compliant with WiMedia standard [1] and the narrow band interferers are generated using MATLAB. Then by using the RF Express software from Tektronix the signal is interfaced into the waveform generator which provides the desired RF signal. Each generated sub-band has an RF bandwidth of $528 \mathrm{MHz}$ with $128 \mathrm{OFDM}$ subcarriers and bit rate of $200 \mathrm{Mb} / \mathrm{s}$. Among the subcarriers 100 carriers carry data. The rest are null, pilot and guard tones. The separation between 
subcarriers is $4.125 \mathrm{MHz}$. The signal also has a cyclic prefix and guard interval of 60.61 and $9.47 \mathrm{~ns}$, respectively. The generated WiMedia sub-bands are presented in Table 1 . The sub-bands follow a simple hopping sequence as $f_{l}, f_{2}, f_{3}$ according to Time Frequency Code (TFC) 1, defined in WiMedia standard so that only one channel exists at any particular time.

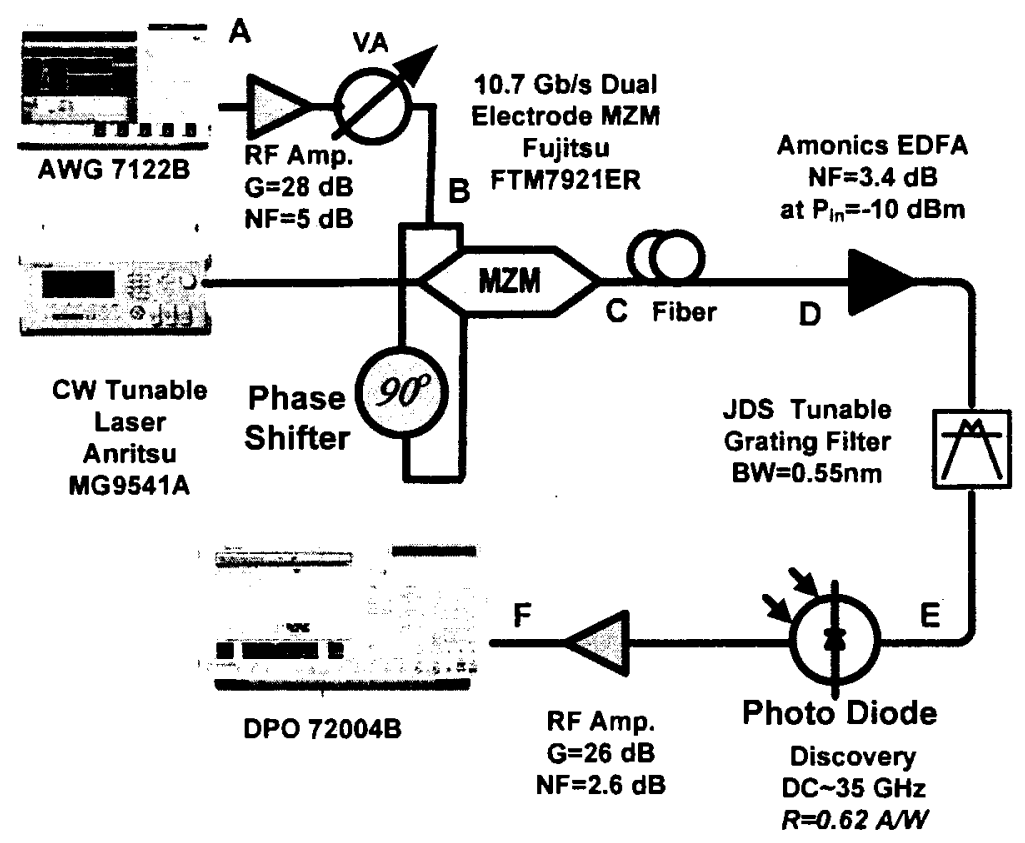

Figure 4.3 Experimental setup for externally modulated MB-OFDM UWB over fiber system under the presence of in-band interferers (NF: noise figure, G: Gain, R: responsivity).

The generated UWB signal has a low power spectral density (PSD) and small footprint. As a result, the UWB signal appears to be very low level background noise to an unintended narrowband receiver. The PSD of the generated signal and interferers (for interferer to UWB peak power ratio of $20 \mathrm{~dB}$ ) at point $\mathrm{A}$ in Fig. 4.3 is given in Table 4.2. 
It is visible from Table 2 that the PSD level difference between UWB and interferer is roughly $20 \mathrm{~dB}$. This difference gives a rough estimation of signal to noise ratio at the narrowband receiver.

Table 4.1 Generated UWB Sub Bands and Corresponding Interferers.

\begin{tabular}{|l|l|}
\hline Band Group & Corresponding in band interferer \\
\hline $\begin{array}{l}\text { Band group 1, 3 sub-bands centered at } f_{l}= \\
\text { 3.432, } f_{2}=3.96 \text { and } f_{3}=4.488 \mathrm{GHz}\end{array}$ & $\mathrm{GHz}$ \\
\hline $\begin{array}{l}\text { Band group 2,3 sub-bands centered at } f_{l}= \\
5.016, f_{2}=5.544 \text { and } f_{3}=6.072 \mathrm{GHz}\end{array}$ & $5 \mathrm{GHz}$ and IEEE 802.11a, WLAN \\
& at $5.8 \mathrm{GHz}$ \\
\hline $\begin{array}{l}\text { Band group } 4,3 \text { sub-bands centered at } f_{1}= \\
8.184, f_{2}=8.712 \text { and } f_{3}=9.240 \mathrm{GHz}\end{array}$ & Marine radar at $8 \mathrm{GHz}$ \\
\hline
\end{tabular}

Table 4.2 Generated UWB Sub-Bands, Interferers and Their Corresponding PSD.

\begin{tabular}{|l|c|c|}
\hline Band Group & $\begin{array}{c}\text { UWB PSD } \\
(\mathbf{d B m} / \mathbf{H z})\end{array}$ & $\begin{array}{c}\text { Interferer PSD } \\
(\mathbf{d B m} / \mathbf{H z})\end{array}$ \\
\hline Band group 1 & -111.48 & -91.53 \\
\hline Band group 2 & -112.35 & $-92.19 /-92.29$ \\
\hline Band group 4 & -118.19 & -98.07 \\
\hline
\end{tabular}


The RF signal is amplified with a broadband RF amplifier from SHF (SHF-810) and a variable attenuator (VA) is used to vary the RF power. A continuous wave (CW) light from tunable laser source (Anritsu MG9541A) has a wavelength of $1550 \mathrm{~nm}$, linewidth of $800 \mathrm{KHz}$, intrinsic relative intensity noise of $-155 \mathrm{~dB} / \mathrm{Hz}$ and output power of $8 \mathrm{dBm}$. The $\mathrm{CW}$ light is injected into a $10.7 \mathrm{GHz}$ dual-electrode Mach-Zehnder modulator (MZM) from Fujitsu, driven by the UWB signal from the output of the arbitrary waveform generator. The MZM has $6 \mathrm{~dB}$ insertion loss and an extinction ratio of $28.5 \mathrm{~dB}$. Using the RF attenuator the RF modulation index $\left(m_{R F}=V_{R F} / V_{\pi}\right)$ of the MZM is kept at $4 \%$ for optimum electrical to optical conversion without causing nonlinear distortion. Here, $V_{\pi}=3.8 \mathrm{~V}$ is the voltage required to induce a $\pi$ phase shift at the MZM.

The average optical power after modulation and before transmission (point $\mathrm{C}$ in Fig. 4.3) is $2 \mathrm{dBm}$. The modulated lightwave is sent through single mode fiber, with fiber loss of $\alpha=0.21 \mathrm{~dB} / \mathrm{km}$ and chromatic dispersion of $17 \mathrm{ps} /(\mathrm{nm} . \mathrm{km})$. We consider UWB over fiber with back-to-back and $20 \mathrm{~km}$ of fiber transmission. After fiber transmission, the UWB signal is optically amplified by an erbium doped fiber amplifier (EDFA) (from Amonics) and then a JDSU tunable grating filter with bandwidth of $0.55 \mathrm{~nm}$ and insertion loss of $3 \mathrm{~dB}$ is used before being detected by a high speed PIN photodiode (Discovery DSC-740 with $3 \mathrm{~dB}$ bandwidth of $35 \mathrm{GHz}$ and responsivity of $0.62 \mathrm{~A} / \mathrm{W}$ ). We used an optical attenuator to keep the input power to EDFA fixed at $-20 \mathrm{dBm}$ and keep the optical signal to noise ratio constant. The EDFA gain is controlled in such a way that the 
insertion loss of all optical components and fiber is compensated at the received power at the photodetector at point $\mathrm{E}$ in Fig. 4.3 is fixed at $4 \mathrm{dBm}$ in all cases. After photodetection the UWB signal is amplified by a broadband RF amplifier from MiniCircuits (ZVA-213) with gain of $26 \mathrm{~dB}$ as shown in Fig. 2. The received signal is evaluated with a high speed real time oscilloscope DPO 72004B from Tektronix.

\subsection{Measures of performance: EVM versus PER}

PER is ratio of the number of received packets in error to the number of transmitted packets. It is a measure of quality of service (QoS) at the receiver. It is notable that a packet will not be transferred to the upper layer even if only one bit is in error. So, PER is a network/ application layer performance. The maximum PER allowable in ECMA-368 standard is $8 \%$ for packet size of 1024 octets with minimum of 100 packets transmitted [4].

The PER depends on the received signal to noise ratio (SNR) and receiver sensitivity. As the power level of the input UWB signal to the MZM increases the transmitted output power increases up to a certain value corresponding to a modulation index $\mathrm{m}_{\mathrm{RF}}=4 \%$ then starts decreasing due to amplitude to amplitude (AM/AM) compression of the MZM. If the SNR increases the packet error rate will decrease. We vary the transmitted UWB power level from $-46.5 \mathrm{dBm}$ up to $-11.13 \mathrm{dBm}$ by changing the UWB input power to the MZM from -32.5 to $3.17 \mathrm{dBm}$ and measure transmitted, 
received RF power and the corresponding PER at the receiver after $1 \mathrm{~m}$ distance of wireless link, shown in Fig. 4.4.

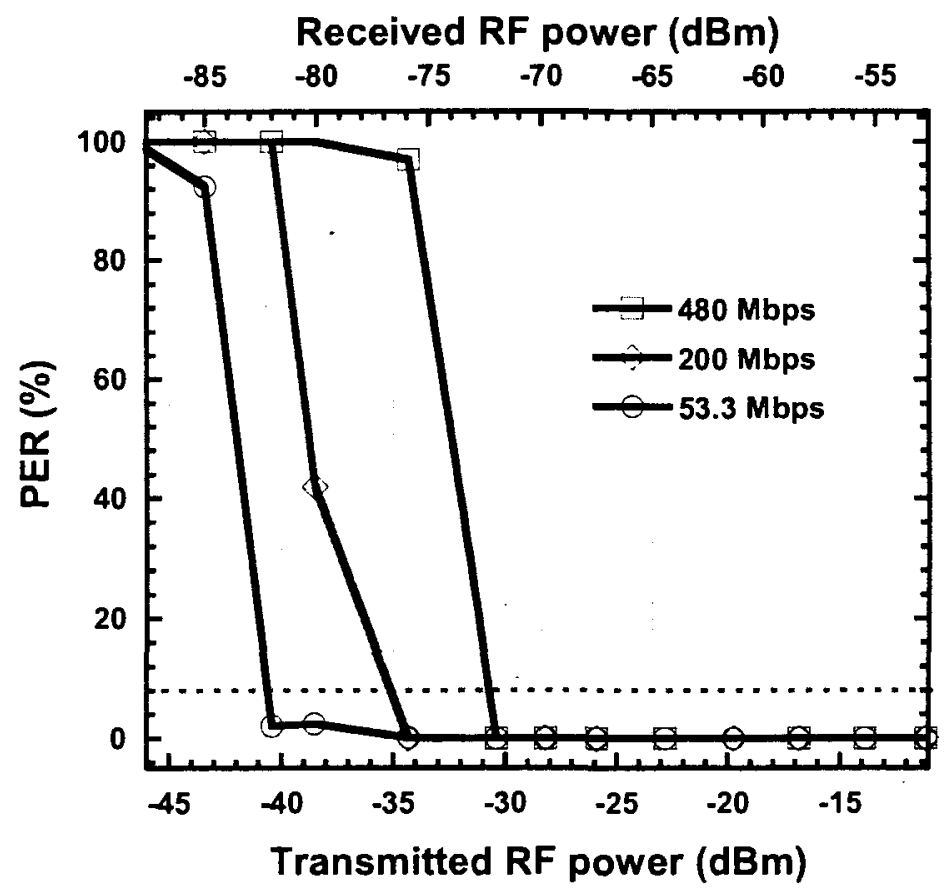

Figure 4.4 PER versus UWB power and data rate for $20 \mathrm{~km}$ optical link and $1 \mathrm{~m}$ wireless link (dotted line corresponds to a PER of $8 \%$ ).

From Fig. 4.4 we found that for maximum allowable PER of $8 \%$ and for $53.3,200$ and $480 \mathrm{Mb} / \mathrm{s}$ the receiver sensitivity is $-82,-76.7$ and $-72.3 \mathrm{dBm}$, respectively, which meet the required receiver sensitivity of $-80.8,-74.9$ and $-70.4 \mathrm{dBm}$ specified by WiMedia physical (PHY) layer. Because the measured UWB receiver sensitivity determines the minimum transmitted power before the transmitting antenna, the required minimum as shown in Fig. 4.5 is $-30.7,-35$ and $-40.5 \mathrm{dBm}$ for 480,200 and $53.3 \mathrm{Mb} / \mathrm{s}$, 
respectively. In addition, it is clear that the receiver sensitivity is worst for the $480 \mathrm{Mb} / \mathrm{s}$. This will limit the dynamic range and wireless transmission range for high bit rates. This is why for $480 \mathrm{Mb} / \mathrm{s}$ the PER performance is poor at low transmitted power levels.

The radiation from a UWB system can cover 2 to $7 \mathrm{GHz}$ of bandwidth. This wide bandwidth operation makes the UWB wireless channel distinct from narrow band channels. The performance will depend on two factors. Firstly, the multi-path reception from the adjacent objects can affect the performance, but UWB signal has an ultra-short duration in time domain, so the number of multi-path components that arrives at the receiver within the period is small and will not have a serious effect as it would have in a narrowband channel [23]. The analysis of the effect of multi-path is rather complex and highly dependent on the environment. As a rule of thumb, the loss increases due to multipath when the distance between the transmitting and receiving antenna is increased. Secondly, the path loss is of an important issue, which at a distance $d$ is given by [24] $P_{L}(d)=\left[P_{L 0}+10 \gamma \log _{10}\left(d / d_{0}\right)\right]+S(d)$, here $P_{L O}=20 \log _{10}\left(4 \pi f_{c} d / c\right) \approx 44 \mathrm{~dB}$ is the path loss for $\mathrm{d}_{0}=1 \mathrm{~m}, f_{c}=\sqrt{f_{\min } f_{\max }}-3.88 \mathrm{GHz}$ where $\mathrm{f}_{\min }=3.168 \mathrm{GHz}$ and $\mathrm{fmax}=4.752 \mathrm{GHz}$ being the lower and upper $-10 \mathrm{~dB}$ cutoff frequencies of the power spectrum of band group 1 for MB-OFDM UWB system, $10 \gamma \log _{10}\left(d / d_{0}\right)$ is the mean path-loss referenced to $1 \mathrm{~m}, \gamma=2$ is the path-loss exponent and $\mathrm{S}(\mathrm{d})$ represents the lognormal shadowing and can be approximated from transmitted and received RF power measurement to $1.85 \mathrm{~dB}$ with a standard deviation of 0.3 . All the values stated are empirical values for a line of sight (LOS) model with omni-directional/omni-directional antenna combination and can vary from location to location [24]. Then the $R F$ power at the receiver $\left(\mathrm{P}_{\text {rec }}\right)$ in terms of transmitted UWB power $\left(\mathrm{P}_{\mathrm{uwb}}\right)$, transmit antenna gain $\left(\mathrm{G}_{T}\right)$ and receive antenna gain $\left(\mathrm{G}_{\mathrm{R}}\right)$ 
can be expressed by $P_{r e c}=P_{u w b}+G_{T}+G_{R}-P_{L}(d)$. For this experiment, we used pair of In4Tel antennas with gain $G_{T}=G_{R}=2 \mathrm{dBi}$. Therefore, from the above equation it is seen that the received power will depend mainly on the mean path-loss referenced to $1 \mathrm{~m}$. To show the performance under a wireless channel, we vary the distance between two UWB antennas in Fig. 4.1 from 1 to $4 \mathrm{~m}$ and observe the corresponding PER at the UWB receiver as shown in Fig. 4.5. From the equation stated before mean path-loss referenced to $1 \mathrm{~m}$ is $6,9.6$ and $12 \mathrm{~dB}$ for 2,3 and $4 \mathrm{~m}$, respectively. As the distance between two antennas increases the packet error rate will aggravate, and this is because the losses are increased and the received signal is attenuated.

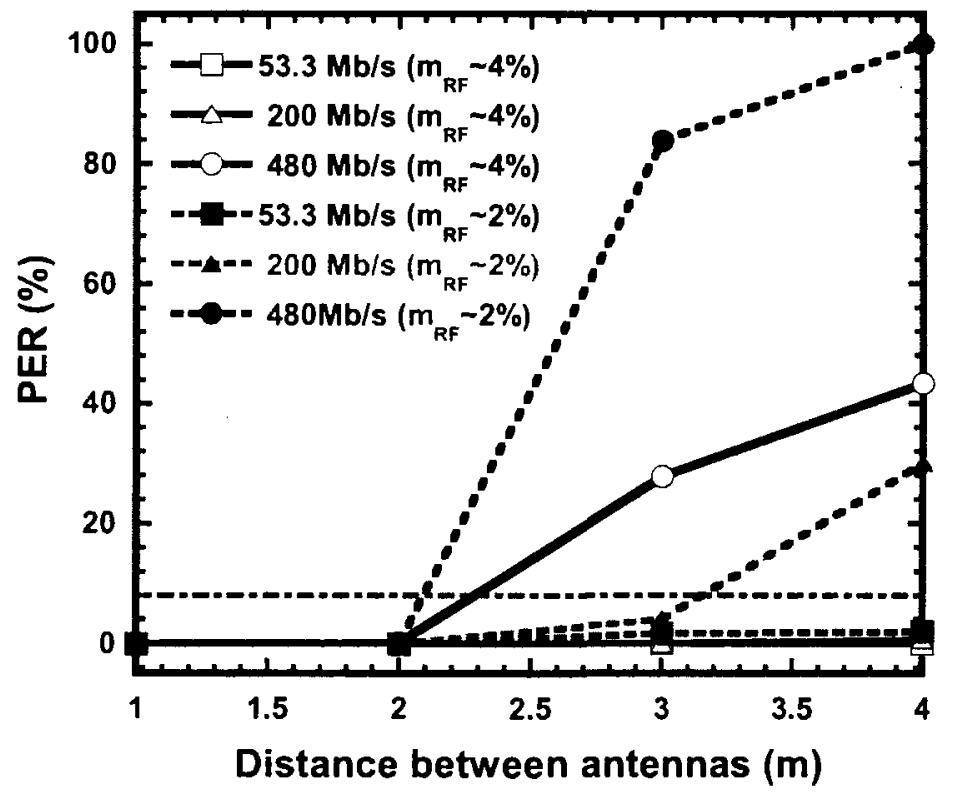

Figure 4.2 PER versus wireless range and data rate for $20 \mathrm{~km}$ of optical link. 
From Fig. 4.5 it is found that PER is almost zero for up to $2 \mathrm{~m}$ wireless at bit rates of up to $480 \mathrm{Mb} / \mathrm{s}$ and input power level of more than $-12.5 \mathrm{dBm}\left(m_{R F}=2 \%\right)$ at the $\mathrm{MZM}$ which corresponds to average transmitted power before the antenna of $P_{u w b}=-26 \mathrm{dBm}$. It is also found that the highest bit rate $480 \mathrm{Mb} / \mathrm{s}$ is the most degraded one by the wireless link length. This is primarily due to the fact that the receiver sensitivity is the worst for this bit rate.

After $4 \mathrm{~m}$ wireless transmission and for typical average transmitted power of -20 and $-26 \mathrm{dBm}$ (corresponding to RF modulation index of $4 \%$ and $2 \%$, respectively) the received RF power using the equation of $P_{r e c}$ is -73.85 and $-79.85 \mathrm{dBm}$, respectively, which is below the receiver sensitivity at 480 and $200 \mathrm{Mb} / \mathrm{s}$, respectively. The corresponding PER can be easily deduced from Fig. 4.6, for example for received RF power of $-73.85 \mathrm{dBm}\left(m_{R F}=4 \%\right)$ the PER is $\sim 45 \%$ and $\sim 0 \%$ for 480 and $200 \mathrm{Mb} / \mathrm{s}$, respectively, while for received $\mathrm{RF}$ power of $-79.85 \mathrm{dBm}\left(m_{R F}=2 \%\right)$ the PER is almost $100 \%$ and $38 \%$ at 480 and $200 \mathrm{Mb} / \mathrm{s}$, respectively. Conversely, at $53.3 \mathrm{Mb} / \mathrm{s}$ the PER is almost $0 \%$ at $\mathrm{RF}$ modulation index of $2 \%$ and $4 \%$. This is because the corresponding received RF power is higher than the receiver sensitivity at this data rate.

EVM or relative constellation root mean square (rms) error is the physical layer (PHY) performance and it contains information about both phase and amplitude errors that are more useful for assessing microwave properties of the signal like the random 
noise, phase noise, AM/AM distortion, AM/PM distortion, delay distortion and interference effects. Average EVM in WiMedia PHY 1.2 standard [4] is computed as follows

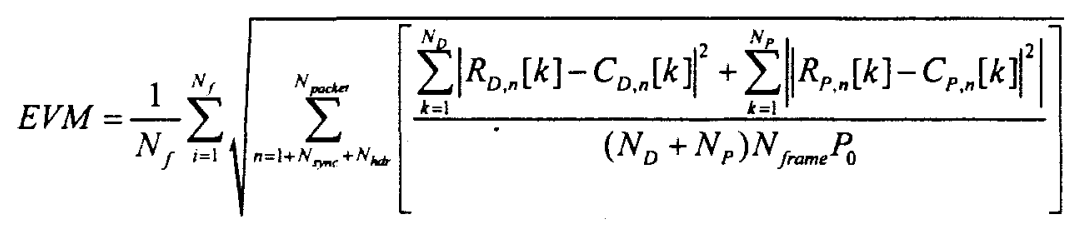

where $N_{f}$ is the number of packets under test, $N_{\text {packet }}$ is the number of symbols in the packet, $N_{\text {sync }}$ is the number of symbols in the preamble, $N_{h d r}$ is the number of symbols in the header, $N_{\text {frame }}=N_{\text {packet }}-N_{\text {sync }}-N_{\text {hdr }}$ is the number of symbols in the frame, $N_{D}$ is the number of data subcarriers, $N_{P}$ is the number of pilot subcarriers, $P_{0}$ is the average power over all payload symbols of the data and pilot constellations, $C_{D, n}[k]$ and $C_{P, n}[k]$ are the transmitted $k^{t h}$ data subcarrier and $k^{t h}$ pilot subcarrier for the $n^{\text {th }}$ OFDM symbol, respectively, and $R_{D, n}[k]$ and $R_{P, n}[k]$ are the observed $k^{\text {th }}$ data subcarrier and $k^{\text {th }}$ pilot subcarrier for the $n^{\text {th }}$ OFDM symbol, respectively.

The EVM required at the transmitter for bit rates of up to $200 \mathrm{Mb} / \mathrm{s}$ is $-17 \mathrm{~dB}$ and above $200 \mathrm{Mb} / \mathrm{s}$ it is $-19.5 \mathrm{~dB}$ respectively with no transmitter attenuation [4]. For conformance testing $-16 \mathrm{~dB}$ is the EVM limit for $200 \mathrm{Mb} / \mathrm{s}$ which is used throughout the paper. The EVM is analyzed on the payload portion of the packet only, over a minimum 
of 100 packets generated from random data. The payload of each packet has to be at least 30 symbols in length. In our experiment each packet consists of 60 symbols. Furthermore, note that PER and EVM are uncorrelated.

But, measuring UWB signals is quite challenging. Firstly, to facilitate the capture of a whole group of time hopped wideband signals, a scope with very wide bandwidth $(>3 \times 528 \mathrm{MHz})$ is required. So, most of the conventional narrowband measuring equipment like electrical spectrum analyzer (ESA) or vector signal analyzers is not compatible with UWB. In addition, a sampling scope [12] cannot be used to measure UWB despite their wide bandwidth. A sampling scope has a limited memory. It accumulates frames over time and gives erroneous results while it is used for measuring the EVM of a continuous signal like UWB. Moreover, at low power, UWB signal is almost in the noise floor of many of the ESAs. So, highly sensitive test equipments are required.

One solution to assess the PHY quality of UWB signals is to use an expensive, wide bandwidth, real time oscilloscope with internal Fast Fourier Transform (FFT) capability to capture the time frames continuously and demodulate using the corresponding software, after doing carrier-phase correction. In this paper a precision high speed real time oscilloscope is used to measure and characterize a MB UWB over fiber system. 


\subsection{Modulation Technique: OSSB versus ODSB}

We compare the OSSB and ODSB, as commonly used optical external modulation techniques. Figure 4.6(a) and (b) shows measured optical spectrum using OSSB and ODSB, respectively. It is well known that OSSB is used to overcome chromatic dispersion caused power fading by suppression of one of the optical sidebands [16]. However, there is a $3 \mathrm{~dB}$ power loss if compared to ODSB. Conversely, ODSB does suffer from power fading specially for back to back because optical sidebands are out of phase and will beat with the optical carrier and add destructively to produce a single RF signal.

Figure 4.7 shows comparison of EVM using OSSB and ODSB for back to back and after $20 \mathrm{~km}$ of fiber transmission. Figure 4.7 shows that the EVM is $-3 \mathrm{~dB}$ or $71 \%$ at data rate of $200 \mathrm{Mb} / \mathrm{s}$ for the back to back with ODSB. At $20 \mathrm{~km}$ the EVM is significantly improved compared to the back-to-back because chromatic dispersion of fiber causes each optical sideband to have different phase shift depending on the fiber length, frequency of RF signal and fiber dispersion and thus the power of the detected RF signal changes. 


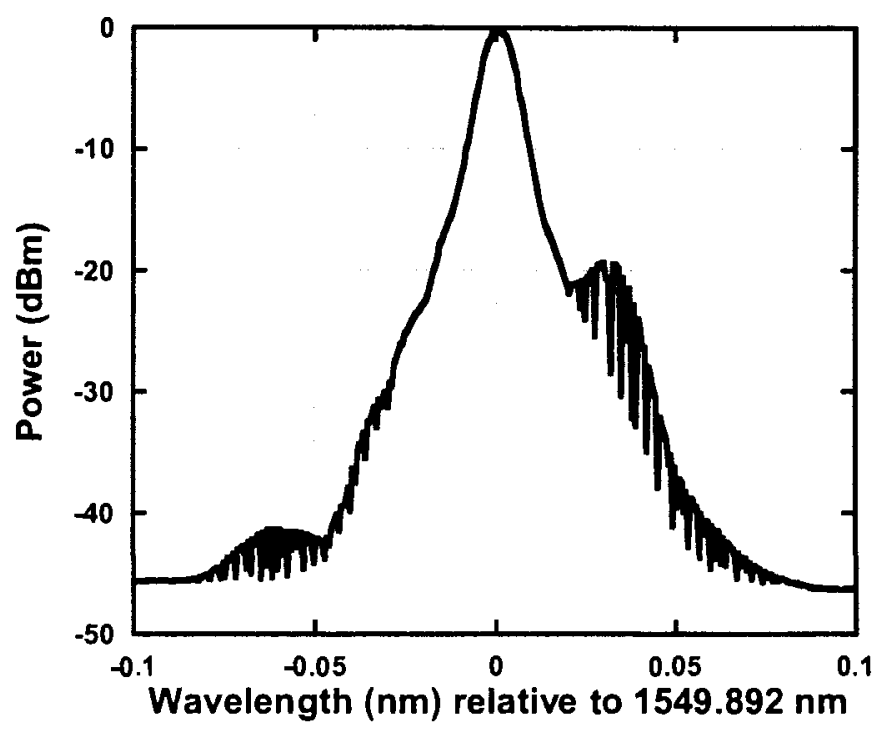

(a)

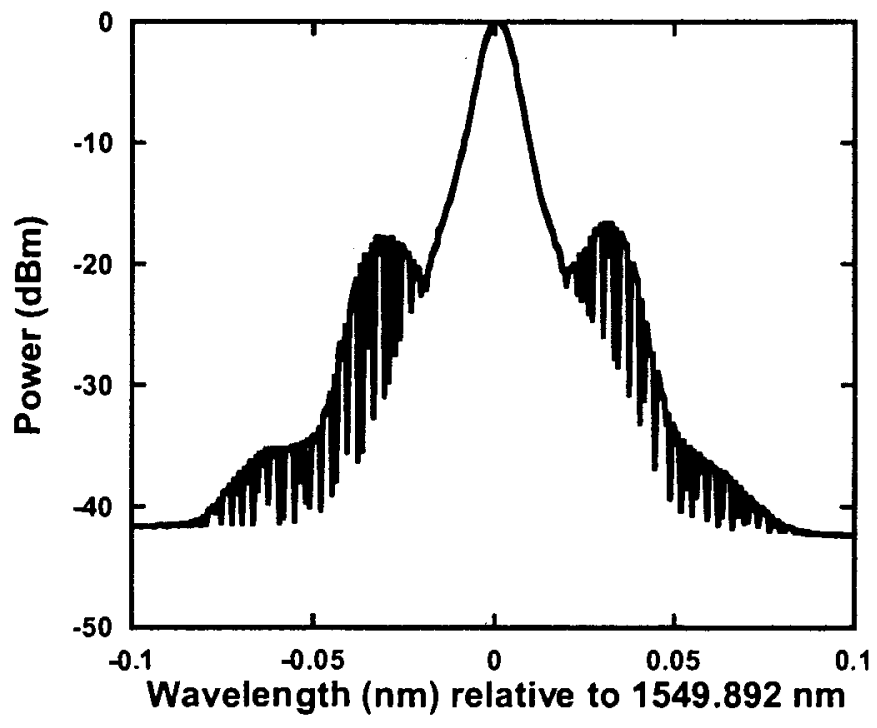

Figure 4.6 Measured optical signal (a) OSSB and (b) ODSB. 


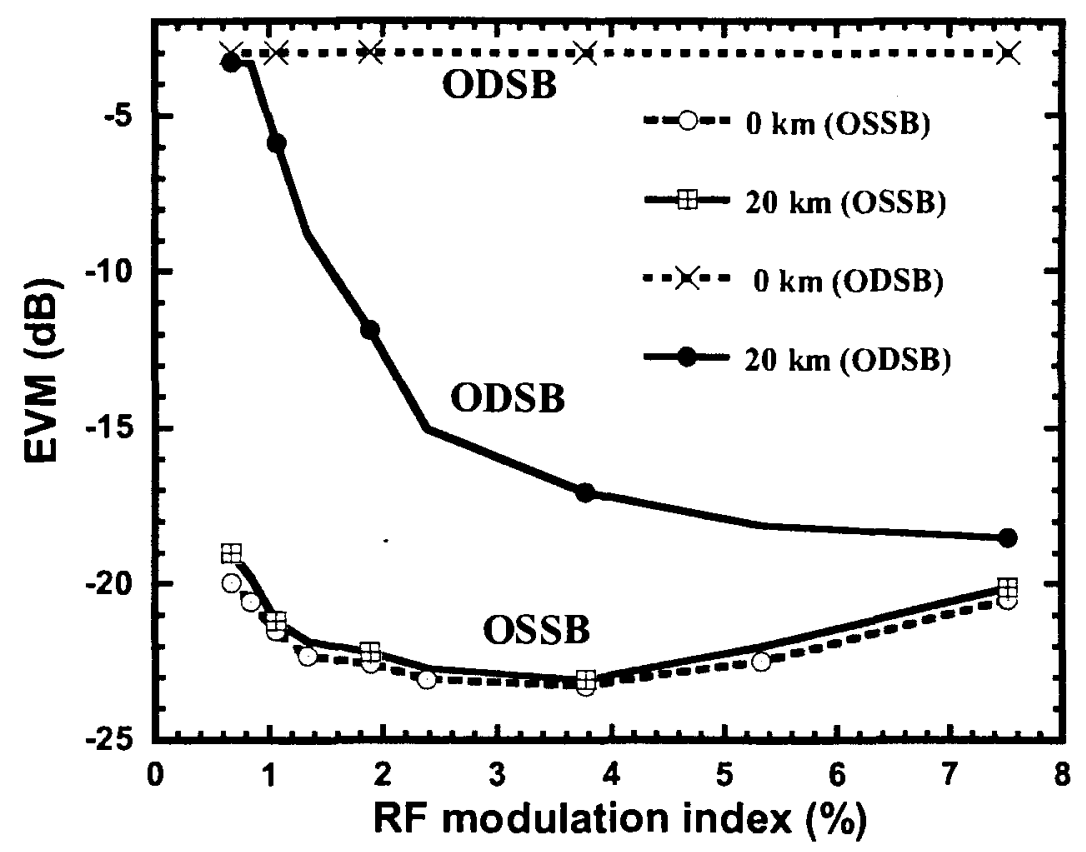

Figure 4.7 Measured EVM using OSSB and ODSB.

In opposition, the OSSB is almost independent of fiber dispersion. As shown in Fig. 4.7 , the EVM is very close and less than $-20 \mathrm{~dB}$ for both back-to back and after 20 $\mathrm{km}$ of fiber transmission. The power fading in ODSB repeats at periodic fiber lengths and can be compensated using different compensation techniques at the expense of increased complexity and cost [16]. It is clearly seen from Fig. 4.7 that OSSB outperforms ODSB at any power level and fiber length. Therefore OSSB modulation is only considered in this paper. 

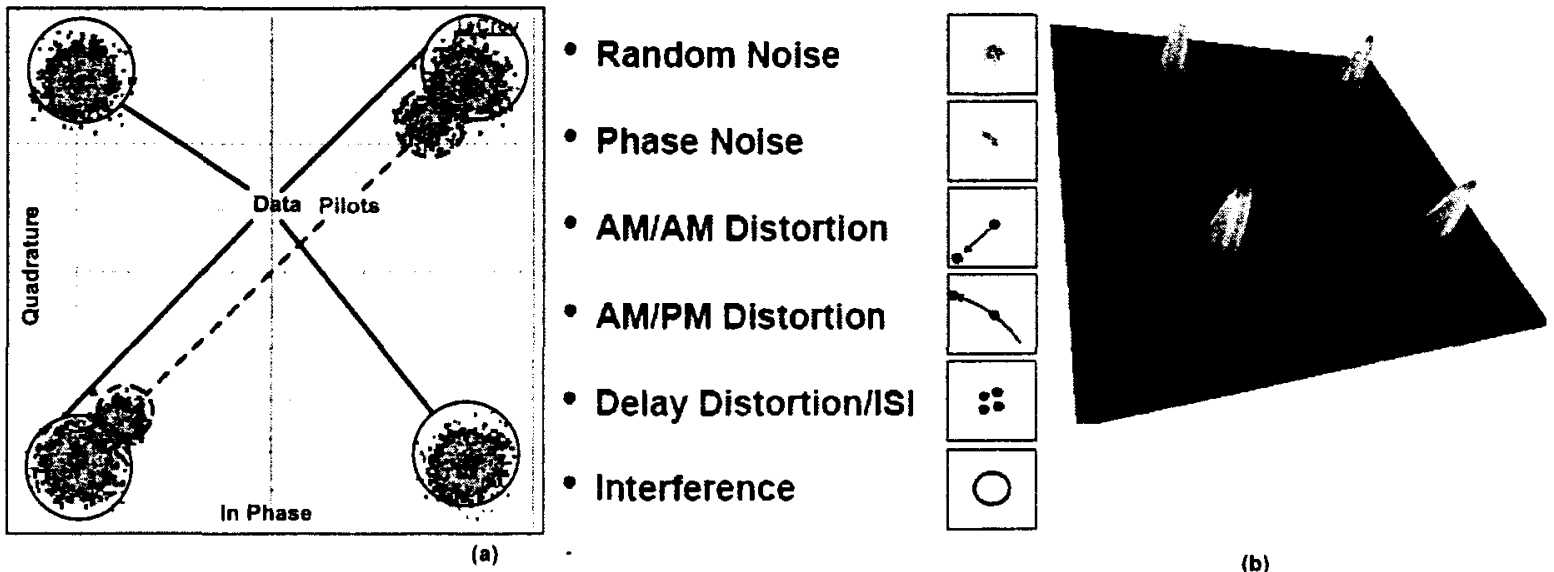

(b)

Figure 4.8 QPSK OFDM constellation in a) two dimension b) three dimension received at the real time oscilloscope for band group 1 after $20 \mathrm{Km}$ of fiber transmission with bit rate of $200 \mathrm{Mb} / \mathrm{s}$ (Colors indicate intensity of power concentration in the received symbol).

With OSSB modulation, the QPSK constellation for data and BPSK constellation for pilots after $20 \mathrm{~km}$ at $200 \mathrm{Mb} / \mathrm{s}$ is presented in Fig. 4.8 (measured with a Lecroy serial data analyzer SDA 11000). The good quality of the constellation is an indication of suitability of the RoF systems for UWB applications.

\subsection{Calculation of EVM for transmission through optical fiber using DE-MZM}

In this section we theoretically analyze MB-OFDM UWB over fiber transmission with OSSB modulation. The common way to generate OSSB is to use a dual electrode 
MZM modulator. However, an MZM modulator is known to be inherently nonlinear in response and may introduce high nonlinear distortion to which OFDM signals are exposed. On the other hand, at the receiver side a narrowband electrical bandpass filter is usually used to filter out the data modulated RF carrier, and the filter determines the optical receiver's response. This filter may have a great impact on the performance of the received data because of its response that may introduce phase and amplitude distortion to which MB UWB OFDM signal is vulnerable. Therefore the theoretical analysis includes the effect of MZM response nonlinearities, fiber dispersion and optical receiver's response. Finally EVM due to fiber dispersion and RF carrier phase noise induced phase distortion is given.

The MB OFDM RF signal $\left(y_{R F}(\mathrm{t})\right)$ is related to the complex baseband signal $\left(x_{k}(\mathrm{t})\right)$ of the $k^{\text {th }}$ OFDM symbol as [2]

$$
y_{R F}(t)=\operatorname{Re}\left\{\sum_{k=0}^{N-1} x_{k}\left(t-k T_{S Y M}\right) V_{R F} \exp \left(j 2 \pi f_{c} t+\varphi(t)\right)\right\}
$$

where $T_{S Y M}$ is the symbol period, $N$ is the number of OFDM symbols, $V_{R F}$ is the driving voltage of the UWB signal, and $f_{c}$ and $\varphi(t)$ is the carrier frequency and phase noise of RF carrier local oscillator, respectively. 
The OFDM symbols $x_{k}(\mathrm{t})$ can be constructed using inverse Fast Fourier transform (IFFT) with a certain coefficients $C_{n}$, which can consist of data symbols, pilots, and training symbols,

$$
\begin{aligned}
& x_{k}(t)=\sum_{n=-N_{S T} / 2}^{N_{S T} / 2} C_{n} \exp \left(j 2 \pi n \Delta f\left(t-T_{C P}\right)\right) \\
& t \in\left[T_{C P}, T_{F F T}+T_{C P}\right], \text { otherwise } 0
\end{aligned}
$$

where, $N_{S T}=128$ is the total number of subcarrier used, $\Delta f=B W / N=4.125 \mathrm{MHz}$ is the subcarrier frequency spacing, $n$ is the subcarrier number, $T_{C P}=60.61 n s$ is the cyclic prefix, $T_{F F T}=1 / \Delta f=242.42 n s$ is the IFFT/FFT period, and $B W$ is the signal bandwidth.

For one OFDM symbol we have

$$
\begin{aligned}
y_{k}(t) & =\operatorname{Re}\left\{x_{k}(t) V_{R F} \exp \left(j 2 \pi f_{c} t+\varphi(t)\right)\right\} \\
& =V_{R F} \operatorname{Re}\left\{\begin{array}{l}
\sum_{n=-N_{S T} / 2}^{N_{S T} / 2} C_{n} \exp \left\{j 2 \pi n \Delta f\left(t-T_{C P}\right)\right\} \\
x \exp \left(j 2 \pi f_{c} t+\varphi(t)\right)
\end{array}\right\} \\
& =V_{R F} \sum_{n=-N_{S T} / 2}^{N_{S T} / 2}\left|C_{n}\right| \cos \left[\omega_{c} t+n \Delta \omega\left(t-T_{C P}\right)+\theta_{n}+\varphi(t)\right]
\end{aligned}
$$

where $\omega_{c}$ is angular frequency of the RF carrier, $\Delta \omega$ is subcarrier spacing and $C_{n}=\left|C_{n}\right| e^{j \theta_{n}}$ is the baseband QPSK signal. 
The MB- OFDM UWB signal is applied to the DE-MZM to modulate a CW lightwave with an optical power $P_{i n}$ and a random phase $\phi(t)$ at a wavelength $\lambda$. For OSSB modulation the DE-MZM is biased at quadrature. The DE-MZM is assumed to have an optical insertion loss of $t_{\mathscr{J}}$. The output optical field from the modulated DEMZM can be written as

$$
E_{\text {out }}(t)=\sqrt{P_{\text {in }} t_{f f} / 2}\left(e^{j \pi \frac{m_{R F}}{\sqrt{2}} y_{k}(t)}+j e^{j \pi \frac{m_{R F}}{\sqrt{2}} \tilde{y}_{k}(t)}\right) e^{j\left(\frac{2 \pi \varepsilon}{i} t+\phi(t)\right)}
$$

where $\mathrm{c}$ is the speed of light in a vacuum, $m_{R F}=V_{R F} / V_{\pi}$ is $\mathrm{RF}$ modulation index, $V_{\pi}=3.8 \mathrm{~V}$ is the voltage required to induce a $\pi$ phase shift at the MZM, and $\tilde{y}_{k}(t)$ denotes the Hilbert transform of $y_{k}(t)$.

After transmission over optical fiber of length $L$, loss $\alpha$ and dispersion $D$, the optical field can be rewritten

$$
\begin{aligned}
E_{o u t}(t) & =\sqrt{2 P_{i n} t_{f f} G e^{-\alpha L}} \exp \left\{j\left(\frac{2 \pi c}{\lambda} t+\phi(t)\right)\right\} \\
& \times \sum_{n_{-N_{S T} / 2}, n_{-N_{S T} / 2+1} \ldots n_{N_{S T} / 2}=-\infty}^{\infty} \cos \left[\left(1+\sum_{k=-N_{S T} / 2}^{N_{S T} / 2} n_{k}\right) \frac{\pi}{4}\right] \\
& \times \exp \left\{-j \frac{\lambda^{2} D L}{4 \pi c}\left(\sum_{k=-N_{S T} / 2}^{N_{S T} / 2} n_{k} \omega_{k}\right)^{2}\right\} \prod_{k=-N_{S T} / 2}^{N_{S T} / 2} J_{n_{k}}\left(\frac{\pi}{\sqrt{2}} m_{R F}\right) \\
& \times \exp \left\{j \sum_{k=-N_{S T} / 2}^{N_{S T} / 2} n_{k}\left[\begin{array}{l}
\omega_{k}\left(t+\frac{L}{v_{g}}\right)+k \Delta \omega\left(\frac{L}{v_{s}}-T_{C P}\right) \\
+\frac{3 \pi}{4}+\theta_{k}+\varphi(t)
\end{array}\right]\right\}
\end{aligned}
$$


where $G$ is the gain of the optical amplifier, $\omega_{\mathrm{k}}=\omega_{c}+k \Delta \omega$ is the $\mathrm{k}^{\text {th }}$ angular frequency subcarrier of the symbol, $J_{n}(\cdot)$ is the $\mathrm{n}^{\text {th }}$ order Bessel function of first kind, $v_{g}$ and $\beta_{2}=-\lambda^{2} D L /(2 \pi c)$ are group velocity and group velocity dispersion coefficient of the fiber, respectively.

After photodetection and electrical filtering, the received $r^{\text {th }}$ subcarrier current of the OFDM symbol can be expressed as Eq (1), where $\mathfrak{R}$ is the responsivity of the photodetector, $H_{e}(\omega)$ is transfer function of the optical receiver, and $\varphi_{r}$ is Gaussian random phase noise with zero mean and variance $\sigma_{p}^{2}$ from the RF carrier. The laser phase noise $\phi(t)$ is cancelled in Eq. (4.7) due to self heterodyne detection.

$$
\begin{aligned}
i_{r}(t)= & -\frac{1}{2} P_{i n} t_{f f} G e^{-\alpha L} \mathfrak{R}\left(\omega_{r}\right) H_{e}\left(\omega_{r}\right) \\
& \times e^{j\left[\omega_{r}\left(1+\frac{1}{v_{g}}\right)-r \Delta \omega T_{C P}+\frac{\pi}{4}+\theta_{r}+\varphi_{r}\right]} \\
\times & \left\{\begin{array}{l}
\sqrt{2} J_{1}\left(\sqrt{2} \pi m_{R F} \sin \left(\frac{1}{2} \beta_{2} L \omega_{r}^{2}\right)\right) \\
\times \prod_{k=-N_{S T} / 2, \neq r}^{N_{S T} / 2} J_{0}\left(\sqrt{2} \pi m_{R F} \sin \left(\frac{1}{2} \beta_{2} L \omega_{r} \omega_{k}\right)\right) \\
+j J_{1}\left(\sqrt{2} \pi m_{R F} \sin \left(\frac{1}{2} \beta_{2} L \omega_{r}^{2}+\frac{\pi}{4}\right)\right) \\
\times \prod_{k=-N_{S T} / 2, \neq r}^{N_{S T} / 2} J_{0}\left(\sqrt{2} \pi m_{R F} \sin \left(\frac{1}{2} \beta_{2} L \omega_{r} \omega_{k}+\frac{\pi}{4}\right)\right) \\
+j J_{1}\left(\sqrt{2} \pi m_{R F} \cos \left(\frac{1}{2} \beta_{2} L \omega_{r}^{2}+\frac{\pi}{4}\right)\right) \\
\times \prod_{k=-N_{S T} / 2, \neq r}^{N_{S T} / 2} J_{0}\left(\sqrt{2} \pi m_{R F} \cos \left(\frac{1}{2} \beta_{2} L \omega_{r} \omega_{k}+\frac{\pi}{4}\right)\right)
\end{array}\right\}
\end{aligned}
$$


Eq. (4.7) shows that each subcarrier will be distorted in amplitude and phase. This distortion results from intrinsic nonlinearities of the DE-MZM response, fiber dispersion and frequency response of the optical receiver.

Considering low RF modulation index of $m_{R F} \ll 1$ and the ideal optical receiver, we can simplify Eq. (4.7) into

$$
\begin{aligned}
i_{r}(t)= & -\frac{1}{2 \sqrt{2}} P_{i n} t_{f f} G e^{-\alpha L} \pi \frac{V_{R F}}{V_{\pi}} \\
& \times\left|C_{r}\right| e^{j\left[\omega_{r}\left(t+\frac{L}{\mathrm{vg}}\right)-r \Delta \omega T_{C P}+\frac{3 \pi}{4}-\frac{1}{2} \beta_{2} L \omega_{r}^{2}+\theta_{r}+\varphi_{r}\right]}
\end{aligned}
$$

Using the approximation of $\omega_{r}^{2} \sim \omega_{c}^{2}+2 r \omega_{c} \Delta \omega$, the received OFDM symbol can be written

$$
\begin{aligned}
y_{k}(t) & \infty \sum_{r=-N_{S T} / 2}^{N_{S T} / 2} i_{r}(t) \\
& \infty \sum_{r=-N_{S T} / 2}^{N_{S T} / 2} \hat{Y}_{r} e^{j r \Delta \omega\left(t+\frac{L}{g_{g}}-T_{C P}\right)}
\end{aligned}
$$

where $\hat{Y}_{r}=X_{r} e^{j\left(-r \beta_{2} L \omega_{c} \Delta \omega+\varphi_{r}\right)}$ is the normalized received symbol corresponding to the transmitted symbol $X_{r}=\left|C_{r}\right| e^{j \theta_{r}}$ on the $\mathrm{r}^{\text {th }}$ subcarrier. Assuming that constant delay will be compensated by cyclic prefix, the error vector magnitude (EVM) can be approximated using [25]

$$
\begin{aligned}
E V M_{0}^{2} & =\frac{1}{N_{S T}} \sum_{k=-N_{S T} / 2}^{N_{S T} / 2}\left|\hat{Y}_{k}-X_{k}\right|^{2} \\
& =\frac{2}{N_{S T}} \sum_{k=-N_{S T} / 2}^{N_{S T} / 2}\left|X_{k}\right|^{2}\left(1-\cos \left(k \beta_{2} L \omega_{c} \Delta \omega+\varphi_{k}\right)\right)
\end{aligned}
$$


Using the following identities

$$
\begin{aligned}
\frac{1}{N} \sum_{k=-N}^{N} e^{j k \theta} & =\frac{1}{N} \frac{e^{j N \theta}-1}{e^{j \theta}-1} \\
& =\exp \left(j(N+1) \frac{\theta}{2}\right) \operatorname{sinc}\left(N \frac{\theta}{2}\right) / \operatorname{sinc}\left(\frac{\theta}{2}\right)
\end{aligned}
$$

And

$$
\int_{-\infty}^{\infty} \cos \varphi_{k} \frac{1}{\sqrt{2 \pi} \sigma_{\varphi}} e^{-\frac{\rho_{k}^{2}}{2 \sigma_{\varphi}^{2}}} d \varphi_{k}=e^{-\frac{\sigma_{\varphi}^{2}}{2}}
$$

where $\left|X_{k}\right|$ and $\varphi_{k}$ are independent random variables. Averaging $E V M_{0} \quad$ we get

$$
\left\langle E V M_{0}^{2}\right\rangle=2\left(\begin{array}{l}
1-\exp \left(-\frac{1}{2} \sigma_{\varphi}^{2}\right) \cos \left(\frac{1}{4}\left(N_{S T}+2\right) \beta_{2} L \omega_{c} \Delta \omega\right) \\
\times \operatorname{sinc}\left(\frac{1}{4} N_{S T} \beta_{2} L \omega_{c} \Delta \omega\right) / \operatorname{sinc}\left(\frac{1}{2} \beta_{2} L \omega_{c} \Delta \omega\right)
\end{array}\right)
$$

The expression given by Eq. (4.13) is the EVM induced by phase distortion. Both RF carrier phase noise and fiber dispersion will introduce relative phase shift between the OFDM subcarriers. Thus inter-carrier interference (ICI) will be induced and OFDM orthogonality will be lost. 
Accounting for other sources of noise such as thermal, relative intensity (RIN), optical amplifier and shot noise, the total EVM can be expressed as [26]

$$
E V M^{2}=\left\langle E V M_{0}^{2}\right\rangle+1 / S N R
$$

where $S N R=\sigma_{s}^{2} / \sigma_{n}^{2}$ is the received signal to noise ratio, with $\sigma_{s}^{2}$ as the received UWB signal power and $\sigma_{n}^{2}$ as the noise power as explained in Appendix B. Fiber dispersion not only induces relative phase shift between OFDM subcarriers but also converts the laser phase noise to RNN. Therefore, SNR in Eq. (4.14) also includes the converted RIN that depends on fiber dispersion. In Appendix A, the converted RIN is analyzed.

\subsection{Simulation Setup}

Figure 4.10 shows simulation setup for MB OFDM UWB with VPI Transmission Maker and MATLAB. First a pseudo random bit sequence (PRBS) is generated which is scrambled into a bit sequence that is free from long strings of simple patterns such as mark and spaces. Then a mother convolutional encoder with coding rate of $1 / 3$ was used. The convolutionaly encoded bits were punctured to achieve coding rate of $5 / 8$. The data 
was bit interleaved and converted into complex valued QPSK sequence according to gray coded constellation. Then for each 100 data, 12 pilots, 10 guard and also 6 null-tones are added to satisfy the $2^{\mathrm{N}}$ condition for radix butterfly algorithm for IFFT [3]. The data is passed through a Saleh-Valenzuela channel for UWB [1]. Then from this data real and imaginary part are separated and written to different text files. All the wireless signal processing is done using MATLAB ${ }^{\mathrm{TM}}$.

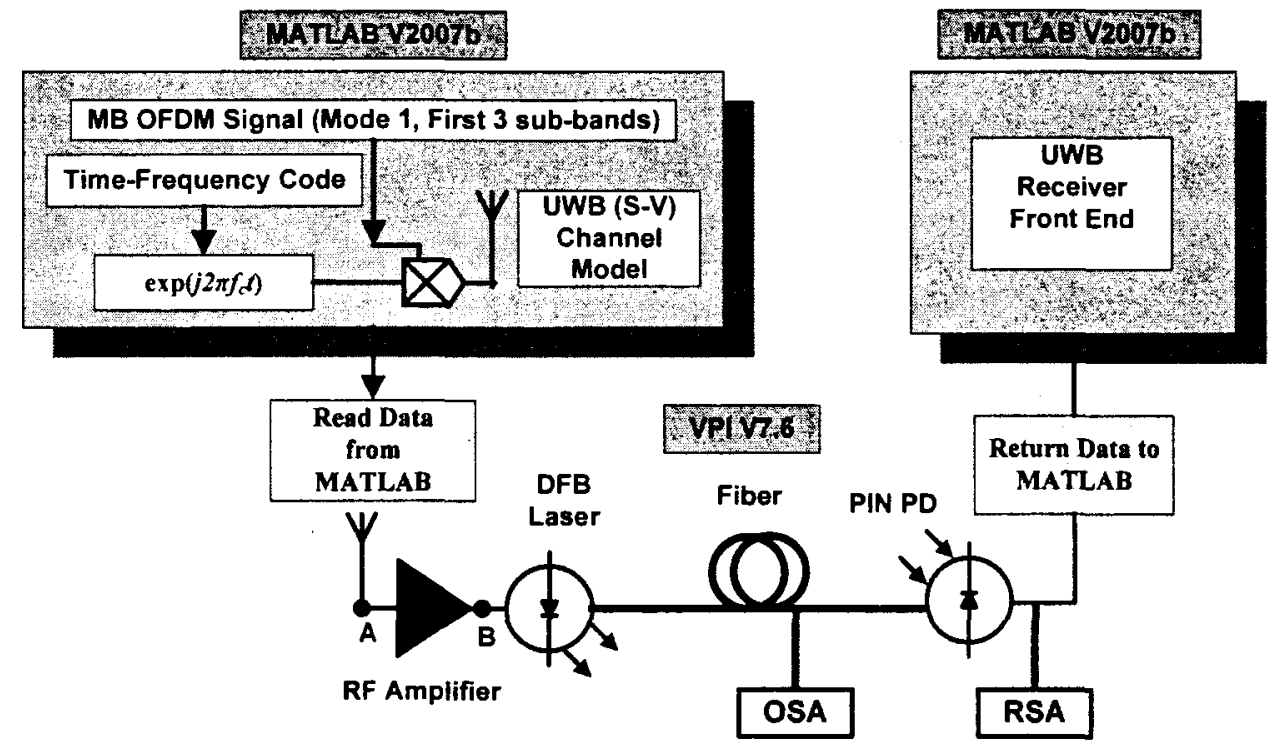

Figure 4.9 Simulation setup for MB OFDM UWB (OSA: optical spectrum analyzer, RSA: RF spectrum analyzer).

This data is read by using file reader of the software VPI7.6 from VPIphotonics $^{\mathrm{TM}}$. A DFB laser is used to directly modulate the signal. The laser has relative intensity noise of $-155 \mathrm{~dB} / \mathrm{Hz}$. The fiber used is a standard single mode fiber 
(SMF) with dispersion of $17 \times 10^{-6} \mathrm{~s} / \mathrm{m}^{2}$, dispersion slope of $0.086 \times 10^{3} \mathrm{~s} / \mathrm{m}^{3}$ and nonlinear refractive index of $2.6 \times 10^{-20} \mathrm{~m}^{2}$. A PIN photodiode with responsivity of $0.62 \mathrm{~A} / \mathrm{W}$ was used as a photo-detector. 


\section{Chapter 5 Results and Discussion}

To make sure that UWB wireless with fiber distribution as shown in Fig. 4.1 satisfies the FCC's spectral requirement, the received signal is tested using the data analyzer. The result from spectral mask test is showed in Fig. 5.1. It is found that the UWB signal passed the spectral mask test and measured adjacent channel power ratio (ACPR) was higher than $20 \mathrm{~dB}$ for RF modulation index of up to $8 \%$ at the MZM. Thus $\mathrm{RF}$ modulation index of up to $8 \%$ is only considered in the following investigation to abide by the FCC regulation.
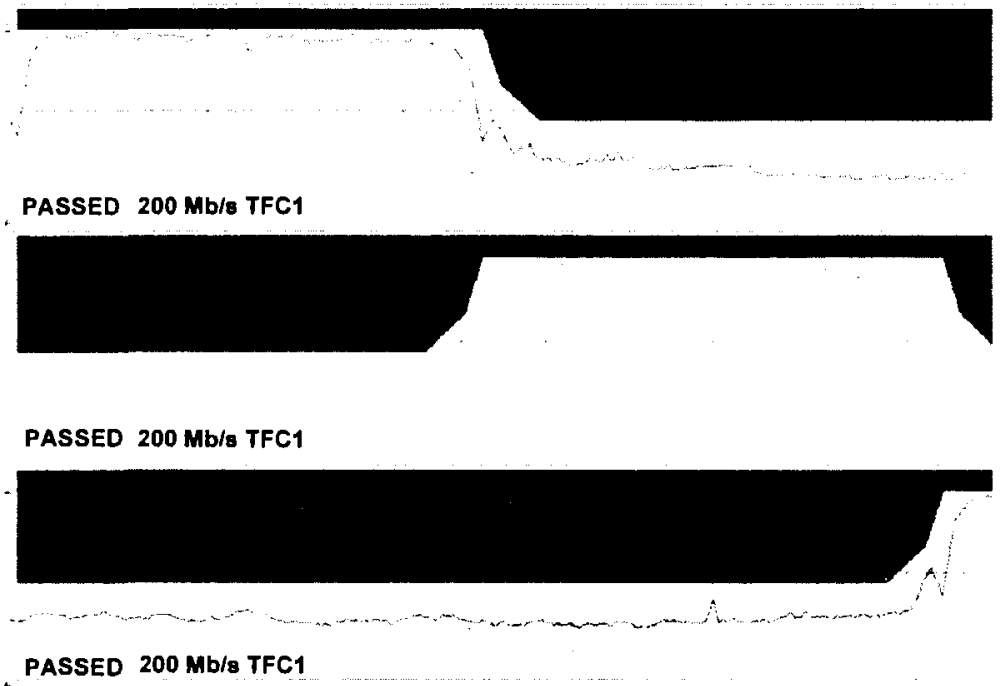

Figure 5.1 Spectral mask test for UWB.

It is well known that there are two optical subcarrier modulation techniques, i.e. OSSB and ODSB. In Section 4.3, we experimentally compared the performance of the two modulation techniques in the system. It was shown that the ODSB modulation cannot 
be used in the MB-OFDM UWB wireless system with fiber distribution. Therefore we only consider the OSSB modulation technique in the following investigation.

In this section we first analyze the impact of optical modulation and fiber transmission. Then the impact of optical demodulation is investigated. Finally we investigate the impact of received optical power on UWB system.

\subsection{Impact of optical Modulation and Fiber Transmission}

Using the setup as shown in Fig. 4.1, we experimentally characterize the impact of optical modulation and fiber transmission using measured EVM. For different fiber lengths, we adjust the gain of the EDFA to fully compensate for all loss and keep the same input power to the photodiode. The ideal optical receiver used has broadband with flat magnitude and linear delay over the considered signal bandwidth. Figure 3 shows measured EVM with RF modulation index for UWB over fiber with fiber length of 0,20 and $40 \mathrm{~km}$, considering bit rate of 53.3 and $200 \mathrm{Mb} / \mathrm{s}$. It is apparent that the minimum EVM is obtained at RF modulation index of $3 \sim 4 \%$ for both 53.3 and $200 \mathrm{Mb} / \mathrm{s}$, almost independent of the bit rate, as shown in Fig. 5.2(a) and (b). At the low RF power the EVM is high due to low SNR. On the other hand, at the high power level the EVM increases due to MZM nonlinearities and fiber dispersion induced nonlinear distortion mainly. 
To fully understand the behaviors in Fig. 5.2, it is required to have full understanding of nonlinear distortion. It is well known that the OFDM signal has a high peak to average power ratio (PAPR). The measured PAPR for the UWB signal is about 14 17 dB from the DV9110 module. Nonlinear distortion for the UWB signal can be induced by RF amplifier due to large PAPR, phase noise of the RF carrier local oscillator due to $\mathrm{PM}$ to $\mathrm{AM}$ (i.e. $\mathrm{PM} / \mathrm{AM}$ ) conversion and nonlinear response of the $\mathrm{MZM}$. However, RF amplifier can induce nonlinear distortion only at higher RF power level. But for OFDM UWB, the RF amplifier is operated in the linear region for most of the time owing to low power spectral density of the UWB. The phase noise may induce nonlinear distortion due to $\mathrm{PM} / \mathrm{AM}$ conversion and created PM/AM modulation is imposed onto the complex waveform.

Nonlinear distortion induced by the nonlinearities of the MZM due to AM/AM modulation and fiber dispersion due to PM/AM conversion is the most important. It is revealed by Eq. (4.7) that the MZM nonlinearities combined with fiber dispersion will induce both $\mathrm{AM} / \mathrm{AM}$ and PM/AM distortion within one symbol.

To distinguish the impact of the MZM response nonlinearities and fiber dispersion, we first consider the back to back UWB over fiber, i.e. without fiber. If the response nonlinearities of the DE-MZM are only considered, Eq. (4.7) is reduced to

$$
\begin{aligned}
i_{r}(t)=P_{i n} t_{f f} G e^{-\alpha L} J_{1}\left(\pi m_{R F}\right)\left[J_{0}\left(\pi m_{R F}\right)\right]^{N_{S T}-1} \\
\times \exp \left\{j\left[\omega_{r} t-r \Delta \omega T_{C P}-\frac{\pi}{4}+\theta_{r}\right]\right\}
\end{aligned}
$$

where the ideal optical receiver response is assumed. 


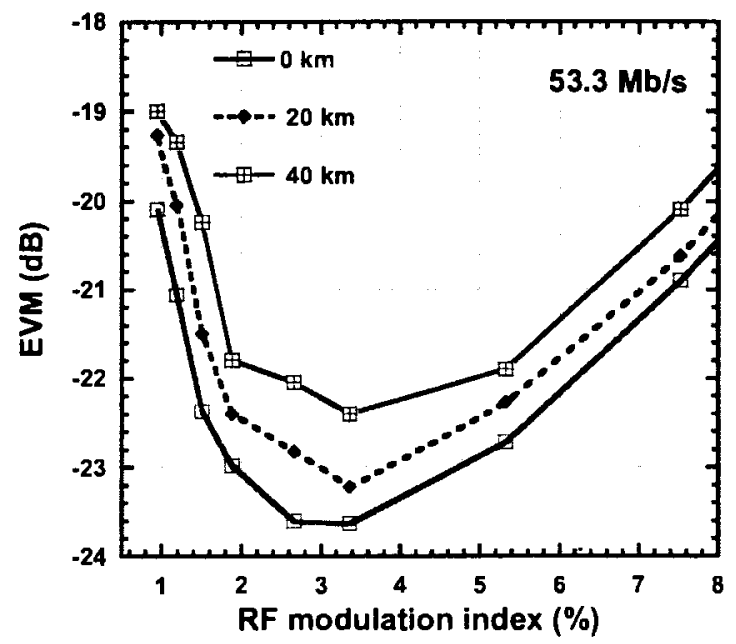

(a)

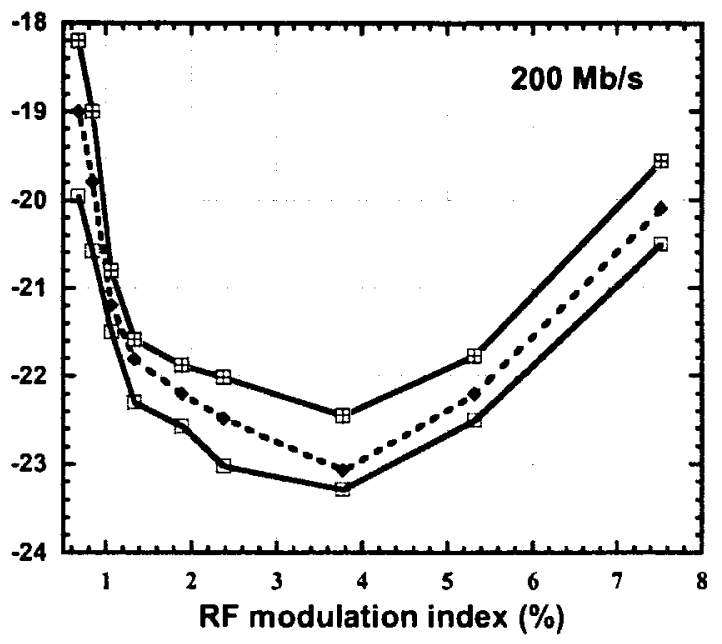

(b)

Figure 5.2 Measured EVM with RF modulation index with a parameter of fiber length for bit rate of (a) $53.3 \mathrm{Mb} / \mathrm{s}$ and (b) $200 \mathrm{Mb} / \mathrm{s}$.

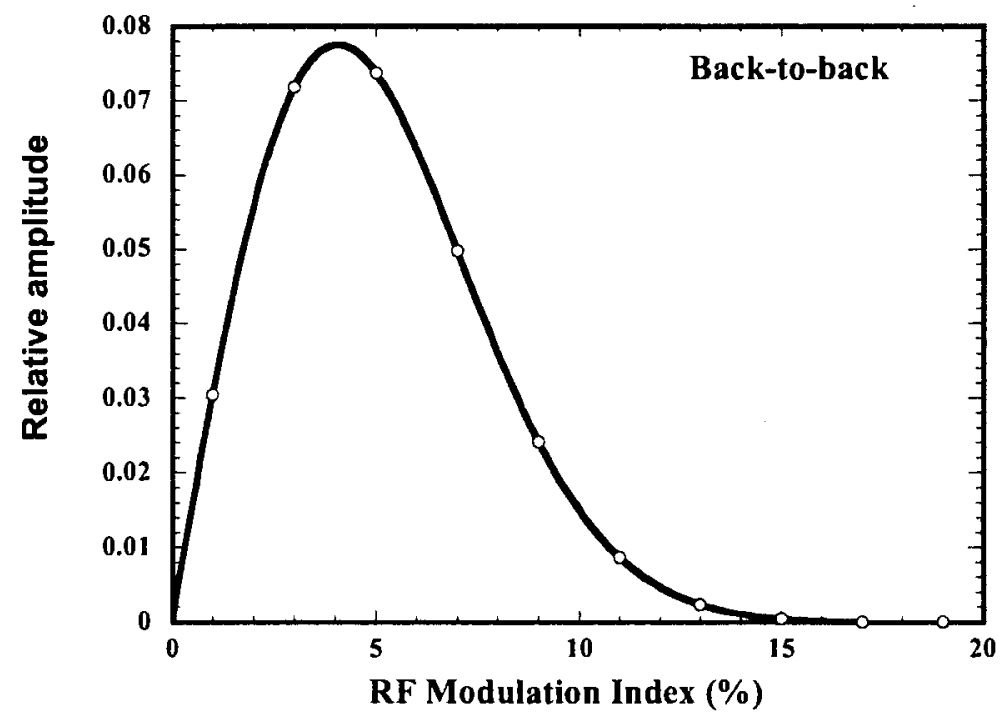

Figure 5.3 Relative amplitude of subcarriers in second band versus RF modulation index for back-to-back UWB over fiber. 
It can be noted from Eq. (5.1), that the received constellation is rotated by $45^{\circ}$. It is also shown that there is a pure AM/AM conversion induced distortion due to the term of $J_{1}\left(\pi m_{R F}\right)\left[J_{0}\left(\pi m_{R F}\right)\right]^{N_{S T}-1}$, which depends only on the total transmitted RF power level at the MZM. Figure 4 shows the relative amplitude (i.e. $\left.2 J_{1}\left(\pi m_{R F}\right)\left[J_{0}\left(\pi m_{R F}\right)\right]^{N_{S \tau^{-1}}}\right)$ of the 128 subcarriers versus RF modulation index for the second band. It is obvious that the amplitude of all the 128 subcarriers is the same for any modulation index, and almost linearly increases with modulation index at RF modulation index of up to $4 \%$. However, RF modulation index of higher than $4 \%$ will decrease the amplitude, i.e. AM/AM compression. Consequently RF modulation index of $4 \%$ is found optimum theoretically. This optimum modulation index of $4 \%$ is almost in good agreement with the above experiment results as shown in Fig. 5.2. However, there is a small discrepancy between the optimum modulation indexes found from Fig. 5.2 and Fig. 5.3. This small discrepancy is mainly due to our simplified approach used in theory where it is assumed that all subcarriers are carrying data, MZM extinction ratio is infinite, and there is no dependence of transmitted power level on bit rate.

In order to investigate the combined effect of fiber dispersion and MZM response nonlinearities on each of the 128 subcarriers, relative amplitude of the subcarriers at 1 , $32,64,96$, and 128 in the second band (centered at $3.96 \mathrm{GHz}$ ) after fiber transmission of 20 and $40 \mathrm{~km}$ is shown in Fig. 5.4, calculated by Eq. (4.7). 


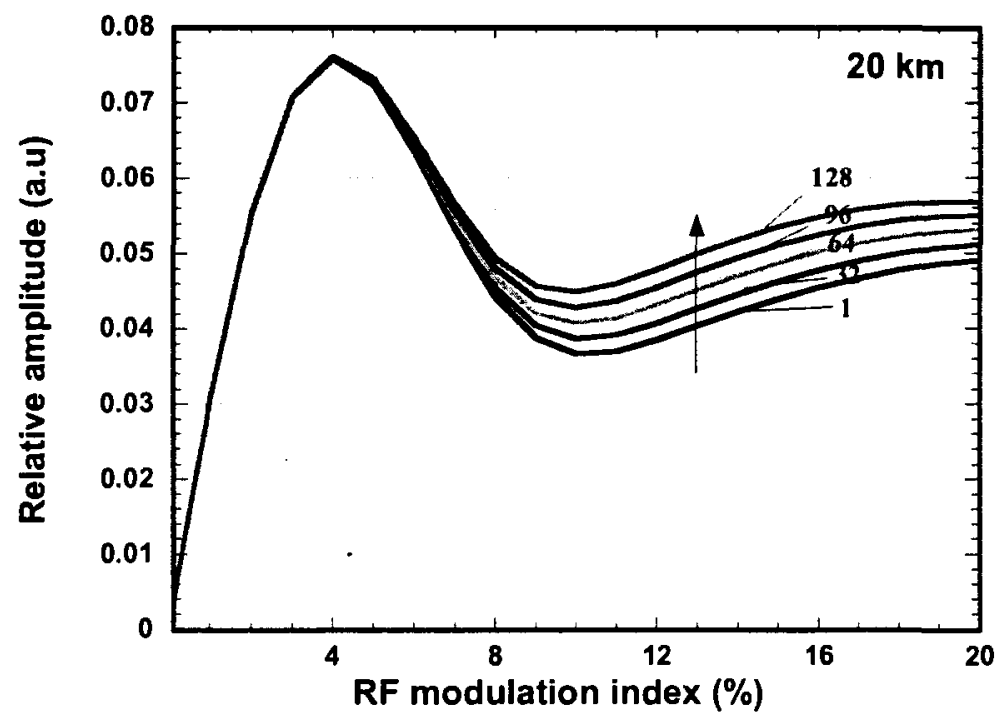

(a)

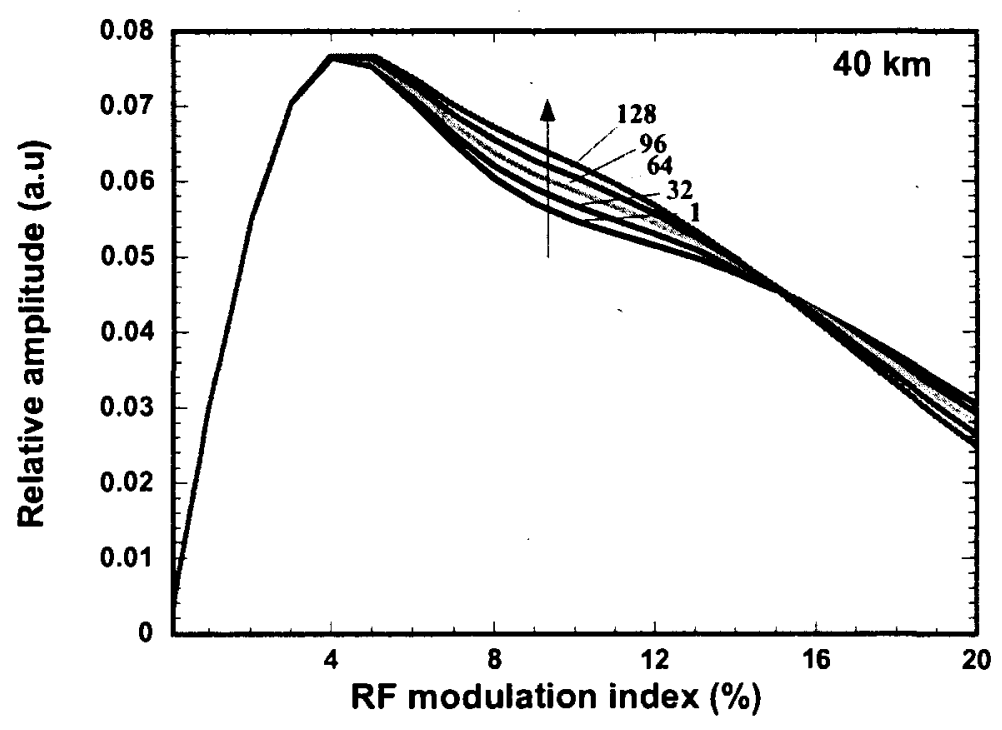

(b)

Figure 5.4 Calculated relative amplitude of subcarriers at 1, 32, 64, 96 and 128 in band 2 versus RF modulation index for UWB over fiber at (a) 20 and (b) $40 \mathrm{~km}$ of fiber. 
It is shown that all the subcarriers have identical relative amplitude that increases almost linearly with the RF modulation index of up to $\sim 4 \%$. However, the subcarriers may not have the same amplitude if modulation index is more than $4 \%$. For example, at the modulation index of $10 \%$, the subcarriers have different amplitudes as shown in Fig. 5.4(a) and (b). This is contrary to the back-to-back transmission as shown in Fig. 5.3. Any amplitude mismatch between subcarriers will distort the received constellation and degrade the EVM.

For the back-to-back UWB over fiber system, the relative phase of the 128 subcarriers is constant and $-45^{\circ}$ for any modulation index. Because fiber dispersion will induce different phase shifts for different subcarriers, the subcarrier phase will depend on fiber length and modulation index. Corresponding to Fig. 5.4, Fig. 5.5 shows the relative phase shift of the subcarriers mentioned above with modulation index. It is seen that the relative phase shift is changed from $-40^{\circ}$ to $45^{\circ}$ and $-30^{\circ}$ to $45^{\circ}$ for 20 and $40 \mathrm{~km}$ of fiber transmission, respectively. Also as expected, different subcarriers have different phase shifts for the same modulation index.

In fact, fiber dispersion induces frequency dependent group delay at each subcarrier. Using Eq. (4.7), the calculated the group delay over the band-two varies by $\sim 0.8$ and $\sim 1.2 \mathrm{ps}$ for 20 and $40 \mathrm{~km}$ of fiber, respectively. 


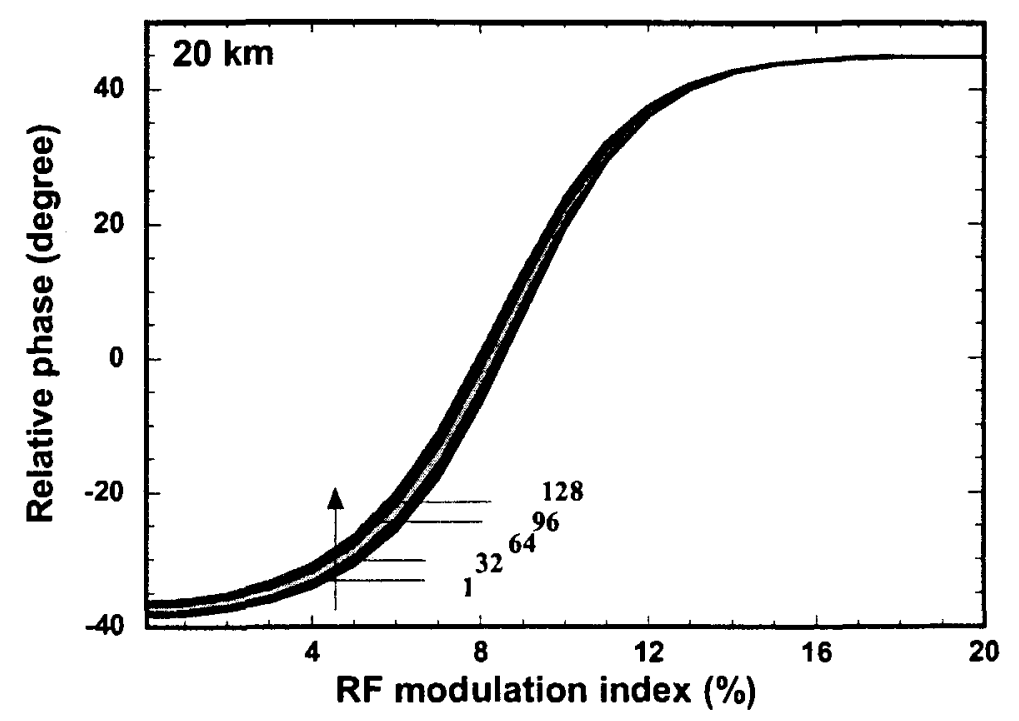

(a)

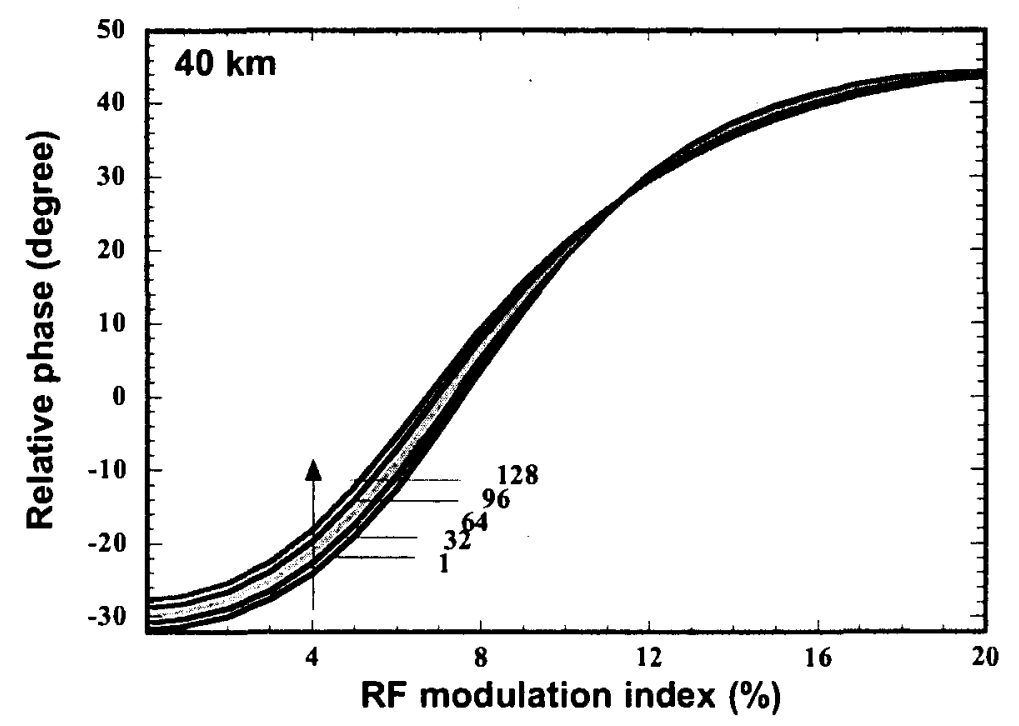

(b)

Figure 5.5 Calculated phase distortion of subcarriers $(1,32,64,96$ and 128) in band-two versus RF modulation index at (a) 20 and (b) $40 \mathrm{~km}$ of fiber transmission. 
Relative phase shift between subcarriers will induce intercarrier interference and result in loss of orthogonality. We then investigate the impact of phase mismatch between subcarriers on the EVM of the system versus fiber dispersion. For RF modulation index of $4 \%$, the amplitude mismatch between subcarriers is negligible. In the EVM computation, we assume that any constant delay can be compensated by cyclic prefix, and the received complex baseband symbol is normalized.

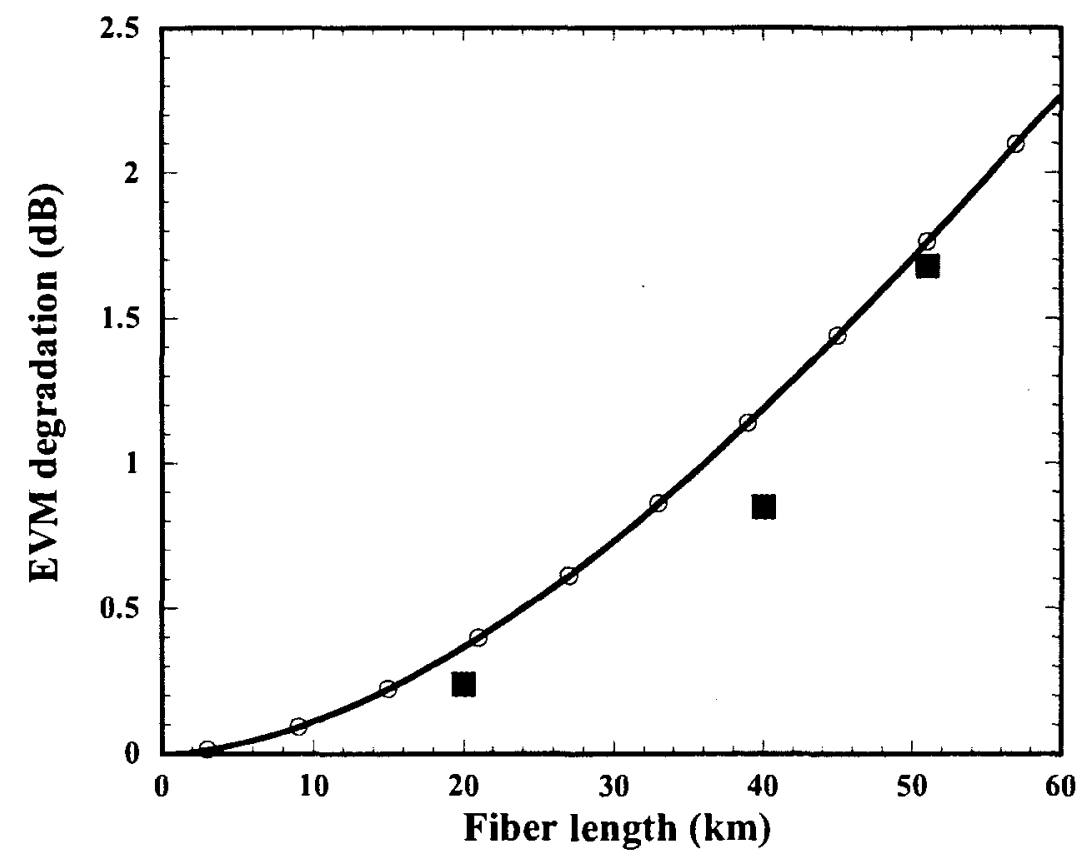

Figure 5.6 Calculated EVM degradation versus fiber length with respect to back to back and 0 $\mathrm{dBm}$ received optical power. Black square: experimental results for 20,40 and $52 \mathrm{~km}$.

Calculated EVM degradation of the system versus fiber length using Eq. (4.14) is shown in Fig. 5.6. In the calculation we used measured EVM of $-24.4 \mathrm{~dB}$ without fiber 
link directly from UWB generator and SNR computed due to thermal noise only, which gives a phase noise power of $\sigma_{\varphi}^{2} \sim 0.0036$. The EVM degradation is obtained with respect to the back to back at modulation index of $4 \%$. Compared to the back to back, it is clearly shown that EVM degradation of $\sim 0.36$ and $\sim 1.1 \mathrm{~dB}$ is expected after 20 and $40 \mathrm{~km}$ of fiber transmission, respectively. This is in good agreement with the measurement in Fig. 5.2. However the experimental EVM is slightly better because of the complex demodulation and error correction schemes used at the UWB receiver. The EVM degradation shown in Fig. 5.6 is due to increased RIN due to the interaction of the laser phase noise and chromatic dispersion as shown in Appendix B, and the phase distortion induced by fiber dispersion as given in Eq. (4.13) Note that for Fig. 5.6, a UWB over fiber system only with a single optical amplifier is considered in order to show the impact of fiber dispersion related penalty.

By the above analysis, we conclude that $\mathrm{RF}$ modulation index of $\sim 4 \%$ is optimum. For modulation index of above $4 \%$, it was found above that MZM response nonlinearities and fiber dispersion induced nonlinear distortion degrades the UWB wireless system performance. This is the reason why the EVM in Fig. 5.2 is increased with modulation index if more than $4 \%$. 


\subsection{Impact of Optical Demodulation}

In this subsection, we will analyze the impact of optical demodulation. We consider two cases: one is the "ideal" optical receiver and the other is bandwidth-limited and has variation of magnitude and time delay over the OFDM signal bandwidth. The bandwidth-limited optical receiver is obtained by inserting a bandwidth-limited electrical filter in the "ideal" optical receiver: Eq. (4.7) shows that the band limited optical receiver response will have great impact on overall performance.

We used a Chebyshev-I bandpass filter centered at frequency, $f_{c}=4 \mathrm{GHz}$ with a 3$\mathrm{dB}$ bandwidth of $3 \mathrm{GHz}$. The magnitude and phase response measured are shown in Fig. 5.7. It is clearly seen from Fig. 5.7(b) that the filter has almost a constant group delay given by the slope of the phase response. However, the response magnitude has a ripple of $\pm 0.5 \mathrm{~dB}$ over the passband. It is expected that the magnitude ripple will induce distortion for the subcarriers of the OFDM signal. We measured EVM for the system with fiber transmission of $20 \mathrm{~km}$, where the two receivers are used. Figure 5.8 shows measured EVM with RF modulation index. We also simulate the UWB over fiber system using VPI-Transmission Maker ${ }^{\mathrm{TM}}$ and MATLAB. The simulated EVM is also shown in

Fig. 5.8. It is seen that a good agreement between the simulated and measured is obtained for using the two optical receivers. However, with ideal receiver response there is discrepancy in EVM performance at higher modulation index due to nonlinearity of the $\mathrm{RF}$ amplifier. It is found that the EVM is degraded by more than $2 \mathrm{~dB}$ at modulation index of $4 \%$ due to using the optical receiver with Chebyshev-I response. Due to the in- 
band ripples of the Chebyshev-I response, some of the sub-carriers of the OFDM signal are distorted in amplitude. Also, the filter's phase or group delay response may cause a slow varying decay trail and can smear the signal at the edges [27]. Smearing will increase the delay spread resulting in inter symbol interference.

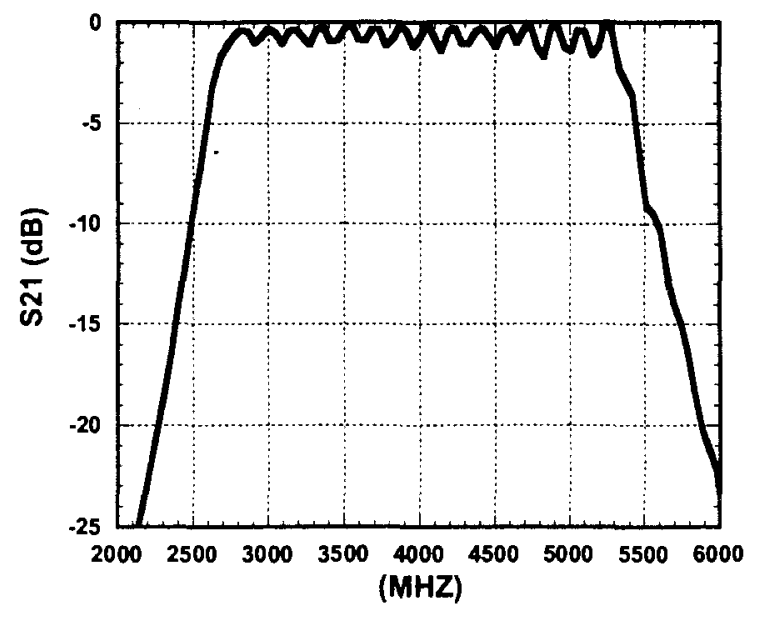

(a)

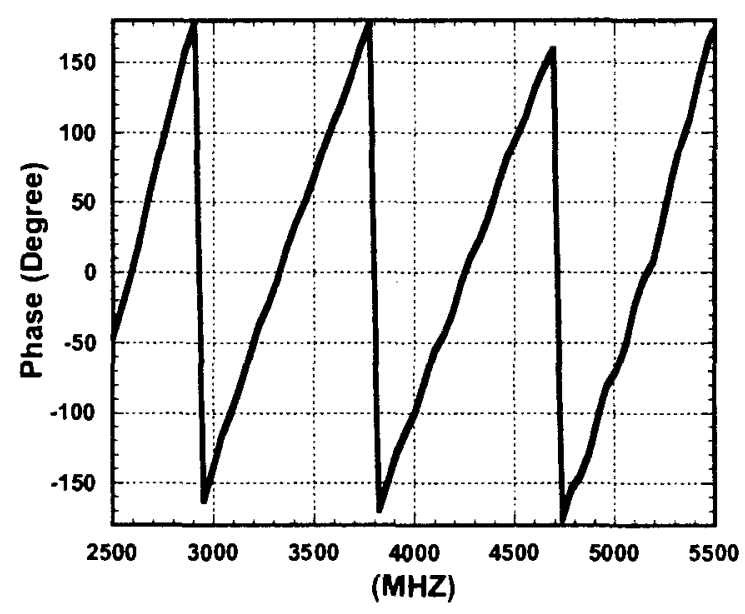

(b)

Figure 5.7(a) Measured magnitude $\left|S_{21}\right|$ and (b) measured Phase response of the experimental filter measured with a HP 8720 vector network analyzer. 


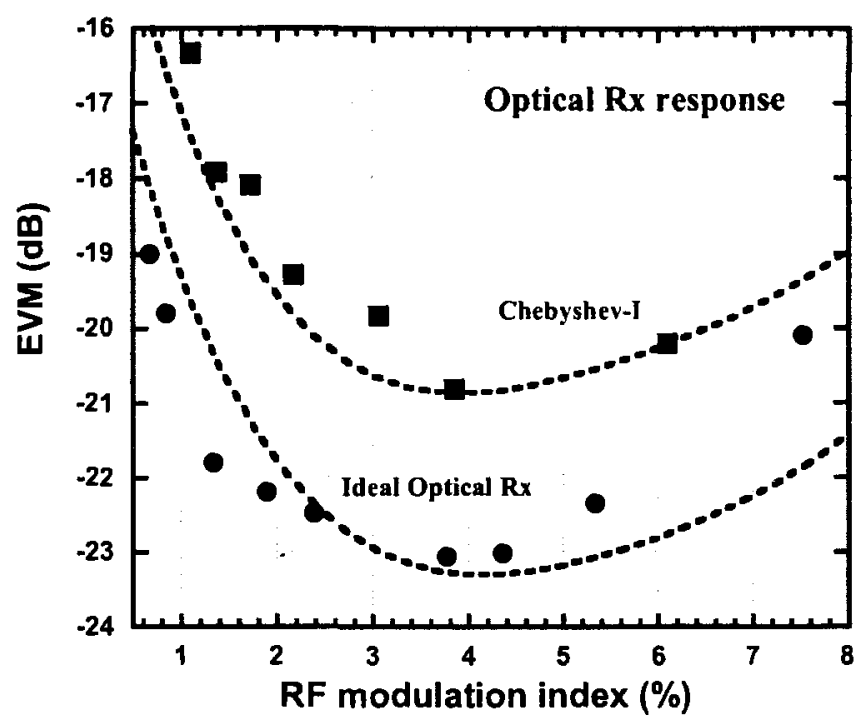

Figure 5.8 Measured (symbol) and simulated (line) EVM using two receivers. Black square: experimental results using optical Rx with Chebyshev-I response, Black circle: experimental results using the "ideal" optical Rx.

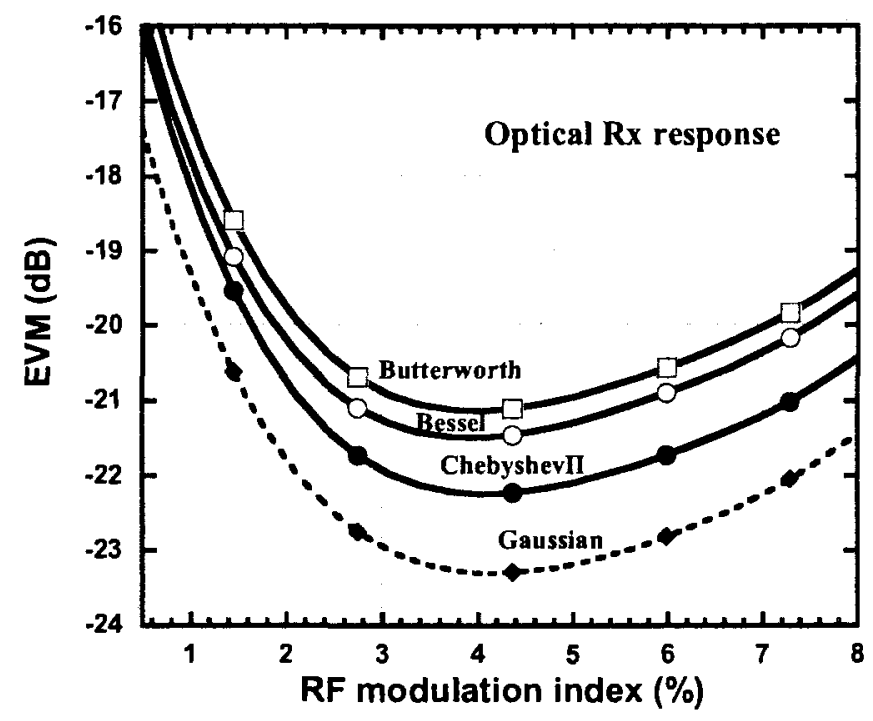

Figure 5.9 Simulated EVM using optical receiver with different responses.

To further understand the impact of optical receiver response, we consider optical receiver with fifth order Butterworth, Bessel, Chebyshev-II, and Gaussian response, 
respectively. Simulated EVM using the above optical receivers is shown in Fig. 5.9. The bandwidth is $3 \mathrm{GHz}$ for all the optical receivers. It is seen that the optical receiver with Gaussian response leads to the best performance. A Gaussian filter with fifth order has flat magnitude and zero delay response in the passband. So, the performance using the optical receiver with Gaussian response will be similar to the ideal receiver. But a Gaussian response is not physically realizable. In Fig. 5.9, the EVM using Butterworth response is $-21.2 \mathrm{~dB}$ compared to $-20.7 \mathrm{~dB}$ using Chebyshev-I in Fig. 5.8 at modulation index of $4 \%$ and it is evident that Butterworth response performs slightly better than Chebyshev-I response of the same order because Butterworth has a flat magnitude response and better delay characteristics than Chebyshev-I filter. To achieve a sharp cutoff, a higher order Butterworth filter is required. But, higher order Butterworth filter will have high overshoot and instability in response compared to Bessel and ChebyshevII filter [28] and lower order Butterworth filter does not fulfill filtering requirements due to its wide passband. Bessel filter's performance is in between Butterworth and Chebyshev-II response since Bessel filter has a linear phase response and excellent impulse response with minimal overshoot within its passband. For a given order, its magnitude response is not as flat as Butterworth and other filters. Also, a Bessel filter requires more complex design and is difficult to integrate with a receiver front end. Consequently it may not be appropriate.

In Fig. 5.9, it is clear that EVM performance using optical receiver with Chebyshev-II response is better than using optical receiver with Chebyshev-I, Butterworth and Bessel response. The response of a Chebyshev-II filter is equiripple in the stopband and 
monotonic in the passband. Also Chebyshev-II's delay response is moderate. In OFDM signal all the subcarriers are independent of each other. As a result, the subcarriers can be added constructively or destructively, resulting in a very large or weak signal, respectively. This is why the OFDM signal has a large PAPR and will suffer badly from nonlinearity. Since OFDM signals are highly sensitive to amplitude distortion, Chebyshev-II response at the receiver front end gives better EVM because the filter will introduce hardly any amplitude distortion. So, Chebyshev-II response is the best choice for optical receiver in UWB over fiber system.

Now, to find out the optimum order and bandwidth, we carry out simulations using Chebyshev-II response with different order and bandwidth. Only odd-orders are considered since even-order Chebyshev filter requires an extra impedance matching network [29]. Simulated EVM with modulation index is presented in Fig. 5.10 for $20 \mathrm{~km}$ of fiber transmission and bit rate of $200 \mathrm{Mb} / \mathrm{s}$. The bandpass filter was centered at $4 \mathrm{GHz}$ and the bandwidth was varied. Figure 5.10 shows that EVM performance is greatly dependent on filter order and bandwidth. The change in EVM can be explained from the magnitude and delay response of the filter which is presented in Appendix C. It is seen that Chebyshev-II has flat magnitude response in the passband. So, only delay response may degrade EVM performance. 


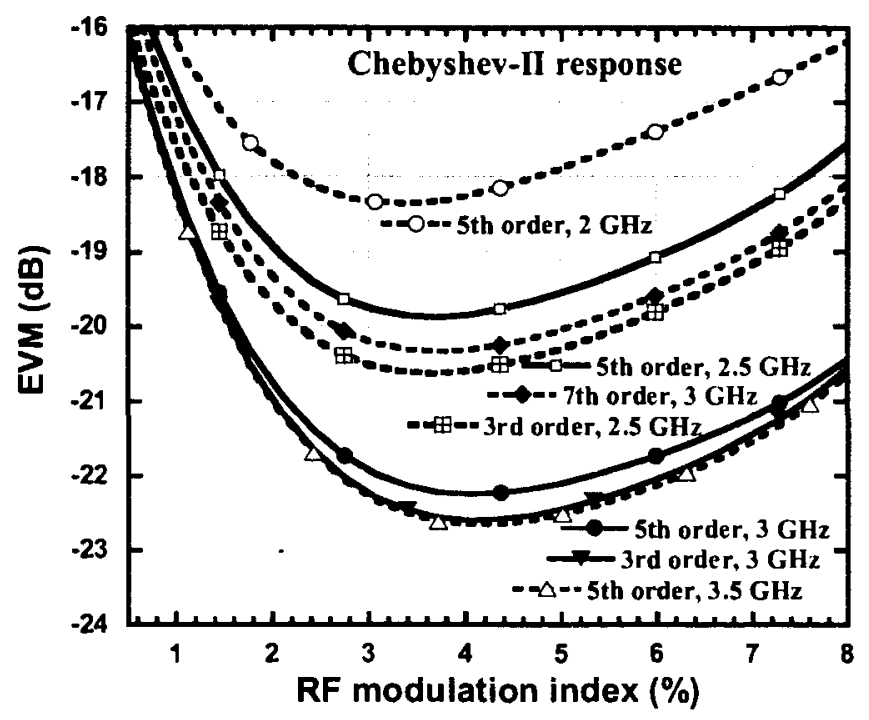

Figure 5.10 Simulated EVM with Chebyshev-II filter order and bandwidth for $200 \mathrm{Mb} / \mathrm{s}$ UWB signal transmitted over $20 \mathrm{~km}$ of fiber.

If the delay fluctuates within the passband, the OFDM subcarriers undergo different phase shift. The effect of delay fluctuation due to filter response is similar to the effect of phase shift introduced by fiber dispersion. A filter's group delay and overshoot are nearly proportional to the filter order and inversely proportional to the filter bandwidth. So, for a higher order filter if the bandwidth is increased the delay response gets better. It is seen that if the bandwidth is low, e.g. $2 \mathrm{GHz}$, the EVM degrades because the OFDM subcarriers near the edges experience a slight attenuation and high group delay fluctuation. The delay within the signal bandwidth varies from 80 to $250 \mathrm{ps}$ for fifth order Chebyshev-II response with $2 \mathrm{GHz}$ bandwidth. In contrast, the average delay fluctuation is 57,80 and 62 ps for third order filter with $2.5 \mathrm{GHz}$, fifth order filter with $2.5 \mathrm{GHz}$ and seventh order filter with $3 \mathrm{GHz}$, respectively. Consequently, the EVM 
performance using seventh order filter with $3 \mathrm{GHz}$ is in between fifth order filter with 2.5 $\mathrm{GHz}$ and third order filter with $2.5 \mathrm{GHz}$. For the 3-dB bandwidth a seventh order filter has a very sharp cutoff but its delay response is not as good as third and fifth order filter. Also microwave filter with higher than fifth order is complex to be implemented and is expensive.

Figure 5.10 shows the EVM performance using third order $3 \mathrm{GHz}$, fifth order 3 and $3.5 \mathrm{GHz}$ filter's response is alike since their average delay fluctuation is close $(42,47$ and 40 ps respectively). On the other hand, the best filter order and bandwidth of the optical receiver in uplink UWB over fiber system may also depend on environment, because an UWB antenna may be placed close to some narrow band interferers like GSM at $0.8 \sim 0.9 \mathrm{GHz}$ and microwave ovens at $1.5 \sim 2 \mathrm{GHz}$. So, for uplink when the data is received by a base station from a user with an antenna under the presence of such narrow band interferers, optical receiver with third order filter response can perform poorly due to its slowly decaying stopband and the large magnitude of out of band ripples. From magnitude response in Appendix B it is seen third order $3 \mathrm{GHz}$ filter will not satisfy the required $20-\mathrm{dB}$ suppression of the sidebands. So, optical receiver with fifth order filter response is a better choice than third order filter response for filtering of MB OFDM UWB signals.

As stated before, increasing the filter bandwidth for a given order improves its delay response, and therefore reduces the EVM. But, the receiver noise increases if the electrical bandwidth of optical receiver is increased which is explained in Appendix B. 
Consequently, an optical receiver with bandwidth as narrow as possible should be selected under the condition of without introducing amplitude and delay distortion. It is also seen from Fig. 5.10 that the EVM performance using the fifth order filter response was improved by only $0.29 \mathrm{~dB}$ at $\mathrm{RF}$ modulation index of $4 \%$ when the bandwidth is increased from $3 \mathrm{GHz}$ to $3.5 \mathrm{GHz}$. Therefore, $3 \mathrm{GHz}$ is considered the optimum bandwidth, which is roughly two times of the bandwidth $(3.168 \mathrm{GHz}=2 \times 3 \times 528 \mathrm{MHz})$ of the band.

\subsection{Impact of Received Optical Power}

Until now, we have assumed all the losses due to fiber and optical components are compensated using an EDFA. To see the effect of the received optical power, we first operate the system for the best EVM at data rate of $200 \mathrm{Mb} / \mathrm{s}$ by setting the RF modulation index to $4 \%$ and received optical power to $0 \mathrm{dBm}$ at the photodetector. Then we insert a precision variable optical attenuator Agilent $8156 \mathrm{~A}$ before the photodetector to vary the received optical.

Figure 5.11 shows measured EVM at $200 \mathrm{Mb} / \mathrm{s}$ considering back-to-back and after $20 \mathrm{~km}$ of fiber. The EVM performance is degraded as the received optical power decreases. To understand the behavior in Fig. 5.11, we study the impairment of noise. In Appendix B, each contribution of thermal noise, shot noise, RIN, signal-ASE beat and ASE-ASE beat noise is studied. It is found that signal-ASE beat noise is dominant in 
additive noise. Therefore, EVM decrease with the increase of the received optical power is due to the fact that UWB RF power is increased with the increase of received optical power. The total EVM dependency on the optical power (given by Eq. (4.14)) is of the form $A / P_{o p t}+\left\langle E V M_{0}^{2}\right\rangle$, A-constant, and $E V M_{0}$ does not depend on optical power. It is clear that EVM degradation is inversely proportional to received optical power.

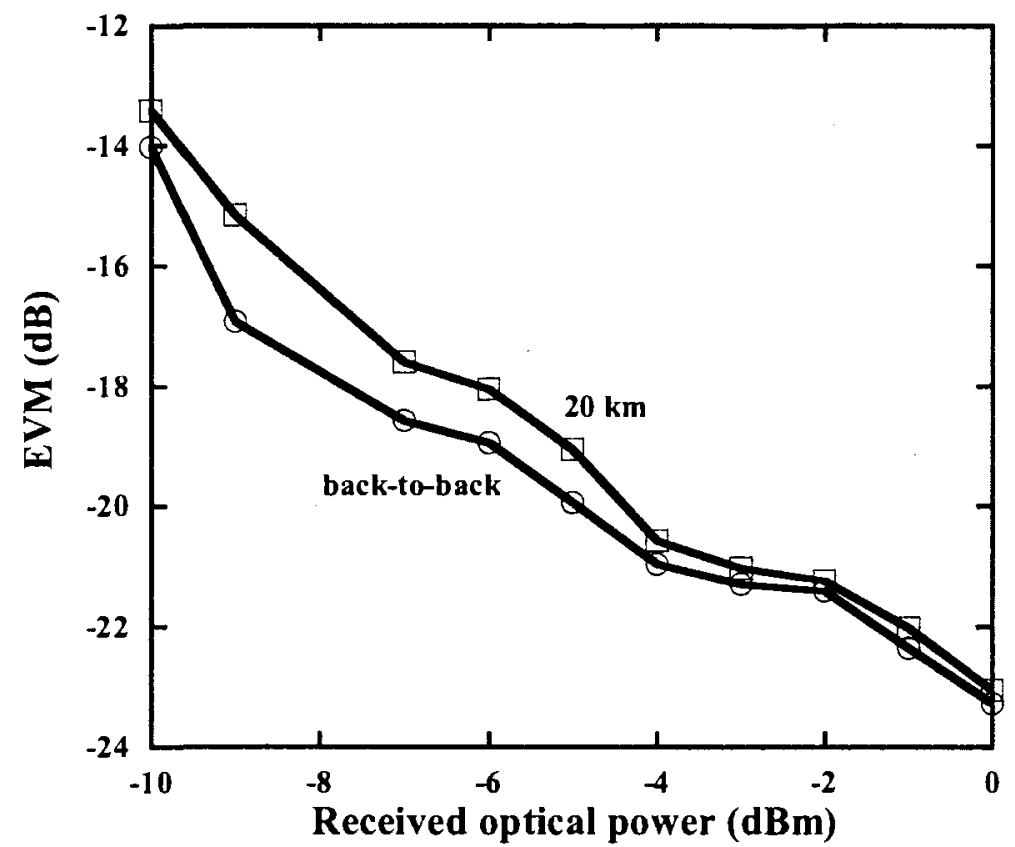

Figure 5.11 Measured EVM versus received optical power at photodetector.

We calculate the EVM degradation versus received optical power as shown in Fig. 5.12. It is evident by Fig. 5.12 that the EVM degrades almost linearly with the received optical power. Experimental results in Fig. 5.11 are in good agreement with calculated EVM from 0 up to $-9 \mathrm{dBm}$. However there is a small discrepancy at low 
optical power and it is due to the limited sensitivity of the real time oscilloscope that has difficulty in triggering at low RF power level. As shown in Fig. 5.11, there is a difference in EVM performance between back-to-back and $20 \mathrm{~km}$ of fiber, which is explained in Fig. 5.6.

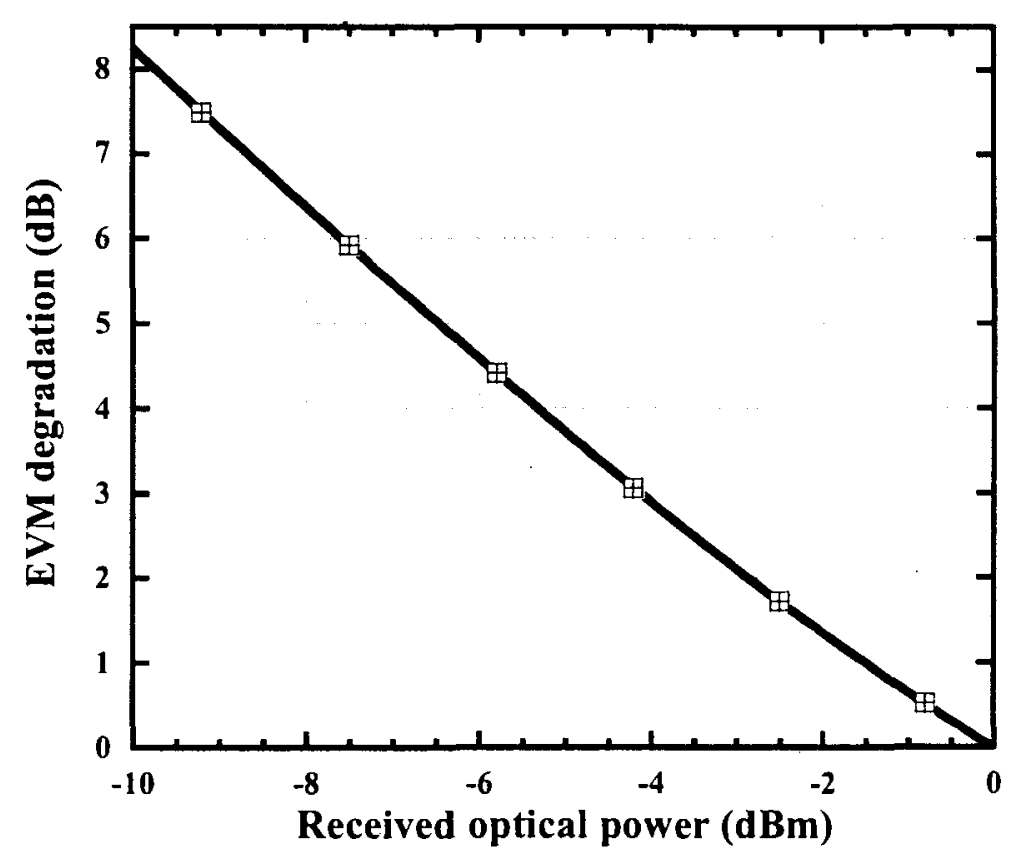

Figure 5.12 Calculated EVM degradation versus received optical power for back-to-back transmission with respect to $0 \mathrm{dBm}$ received optical power.

Another important finding from Fig. 5.11 is that the received optical power required in UWB over fiber is higher compared to conventional radio over fiber which can work with optical power of as low as $-40 \mathrm{dBm}$ [16]. The higher optical power 
requirement is due to the low RF modulation index and the low power spectral density ($41.3 \mathrm{dBm} / \mathrm{MHz}$ ) of the UWB RF signal.

\subsection{Impact of Relative Intensity Noise}

In this section we discuss the impact of various physical parameters that control laser RIN and fiber transmission with focus of MB UWB technology. Laser RIN depends on many quantities, the most important of which are power at laser output, relaxation frequency, frequency of the RF signal, magnitude of the optical feedback, mode suppression ratio and temperature.

\subsubsection{Laser Intrinsic RIN, Resonant Frequency and Laser Output Power}

Ultra wide band systems are adversely affected by the RIN in two ways. Since the transmitted RF signal has a wide bandwidth the total noise over signal bandwidth is large. On the other hand most of the low cost diode lasers' relaxation frequency coincides with the frequency spectrum of the MB UWB signal. We will first investigate how laser intrinsic RIN affects the system performance and how we can improve system performance by reducing intrinsic laser RIN. Using the setup as shown in Fig. 4.1, we experimentally characterize the impact of relative intensity noise and fiber transmission using measured error vector magnitude (EVM). We adjust the gain of the EDFA to fully compensate for all loss and keep the same input power to the photodiode. 
Measured RIN spectrum versus frequency is presented in Fig. 5.13 at the laser output for the laser module in Fig. 4.1 with linewidth of $30 \mathrm{MHz}$. It is observable that RIN frequency spectrum is not flat. The RIN spectral density is small at low frequencies and peaks at the relaxation resonance frequency and then falls to the shot noise level. Measured RIN peak frequency and corresponding intensity noise spectral density is presented in Fig. 5.14. It is evident from Fig. 5.14 that as the laser output power increases, the RIN peak shifts to higher frequency and the RIN magnitude is decreased. System's EVM performance as a parameter of laser output power for back to back transmission is presented in Fig. 5.15. We found that EVM performance depends highly on the RIN of the laser.

At low power levels, i.e. from -6 to $-2 \mathrm{dBm}$, EVM performance of all three bands is poor due to high RIN of the laser. Also at low power the RIN peak resides at low frequencies. That is why the first band $(3.432 \mathrm{GHz})$ of MB UWB suffers most at low power and has poor EVM performance. We see that EVM peaks at around $-3 \mathrm{dBm}$ which makes perfect sense since at around $-3 \mathrm{dBm}$ the RIN peak exactly coincides with the first band. As the laser output power increases from -2 to $0 \mathrm{dBm}$ the relaxation peak shifts to the second band and worst EVM performance for second band $(3.96 \mathrm{GHz})$ is observed at $-1 \mathrm{dBm}$. After $-1 \mathrm{dBm}$ with the increase of laser power the RIN begins to decrease and falls below $-142 \mathrm{~dB} / \mathrm{Hz}$. That is why EVM performance of all the bands improves considerably after $0 \mathrm{dBm}$. The RIN peak eventually shifts to the third band $(4.488 \mathrm{GHz})$ at around $1 \mathrm{dBm}$ so its EVM performance is worse than the other bands. However, it is 
notable that since this peak occurs at higher power with low RIN, overall EVM performance of all the bands is satisfactory after $1 \mathrm{dBm}$.

Therefore, choice of laser output power is a critical factor in minimizing system's intrinsic RIN and avoiding operation exactly at the RIN peak frequency. Since, most of the low cost commercial diode lasers have an output power of $2 \sim 3 \mathrm{dBm}$ and relaxation peak at around $5 \mathrm{GHz}$. So we choose the laser output power of $2 \mathrm{dBm}$ in the rest of thesis.

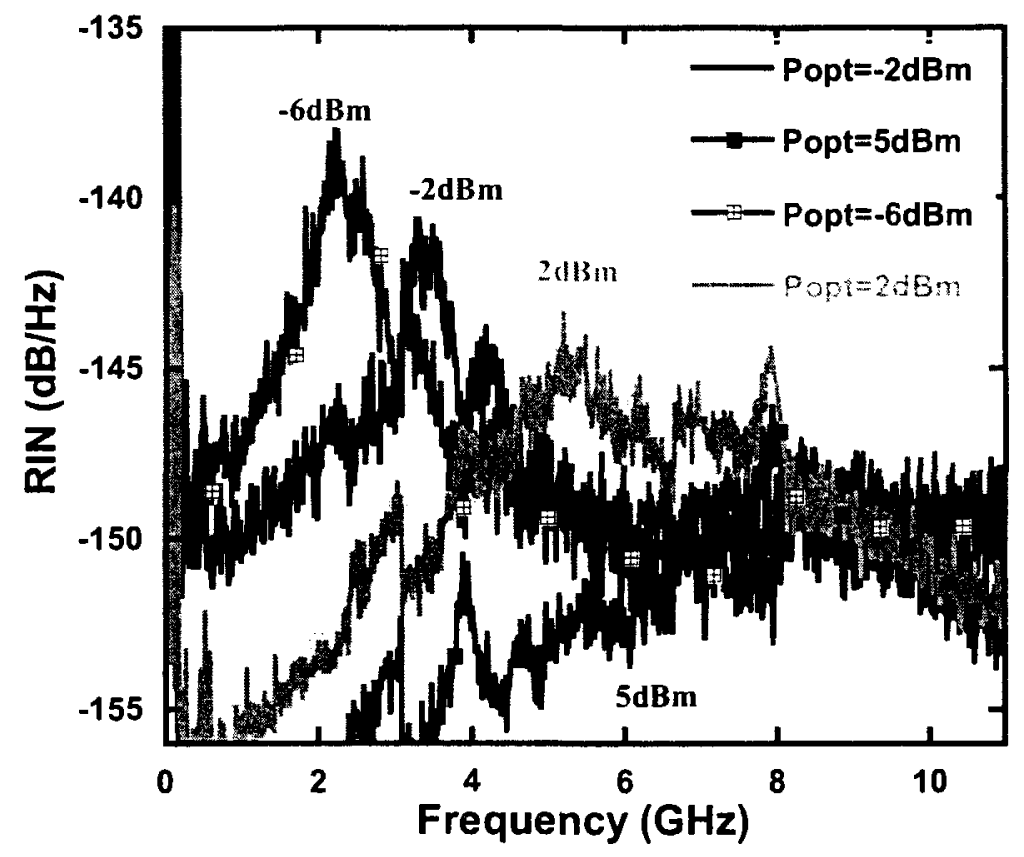

Figure 5.13 Measured spectral density of RIN as a function of frequency for back to back transmission. 


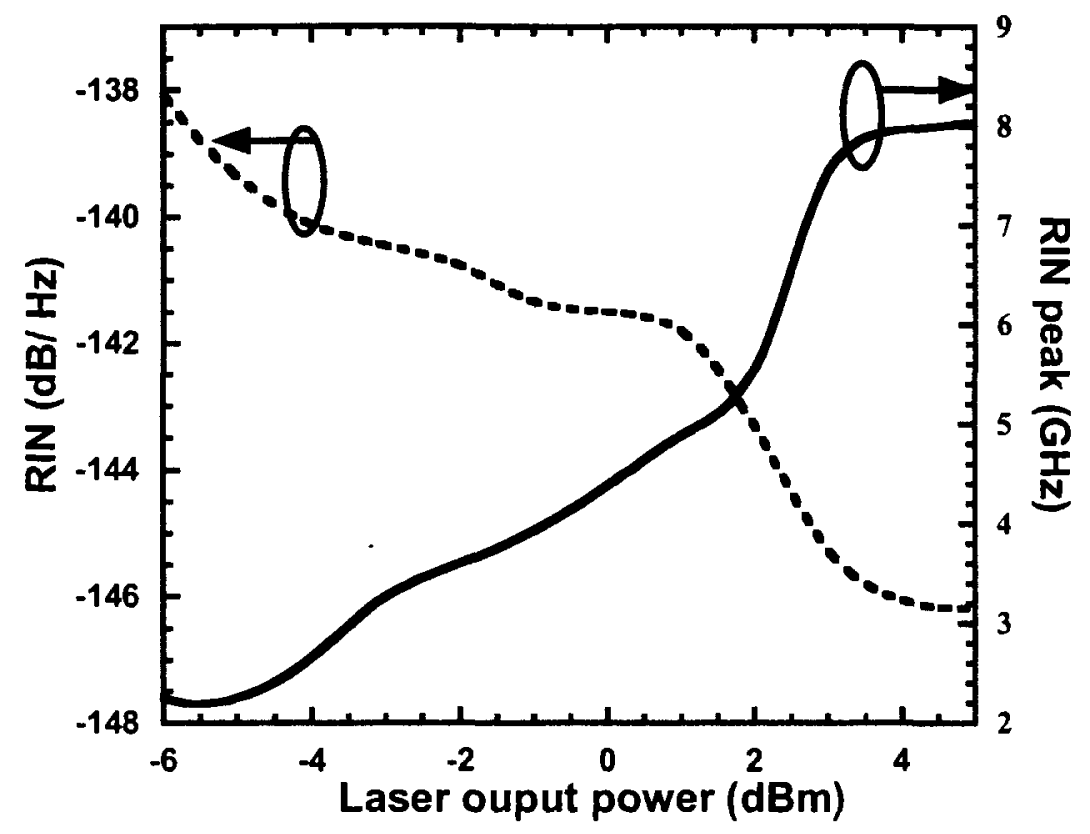

Figure 5.14 Measured RIN peak frequency and corresponding spectral density of RIN for back to back transmission.

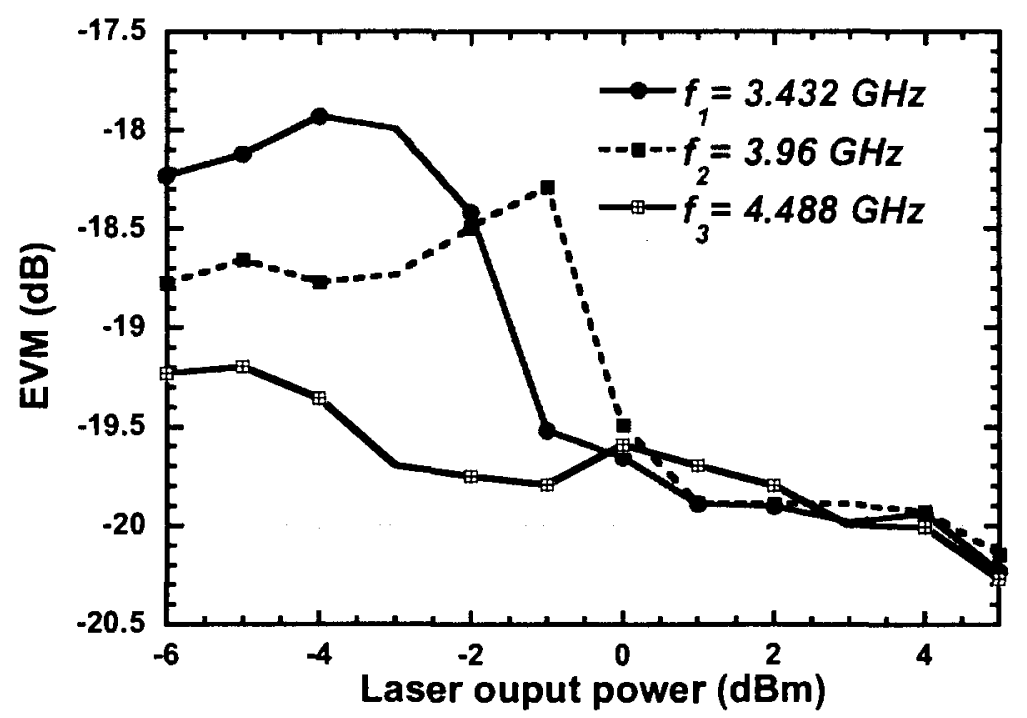

Figure 5.15 Measured EVM with laser output power for back to back transmission at bit rate of $200 \mathrm{Mb} / \mathrm{s}$. 


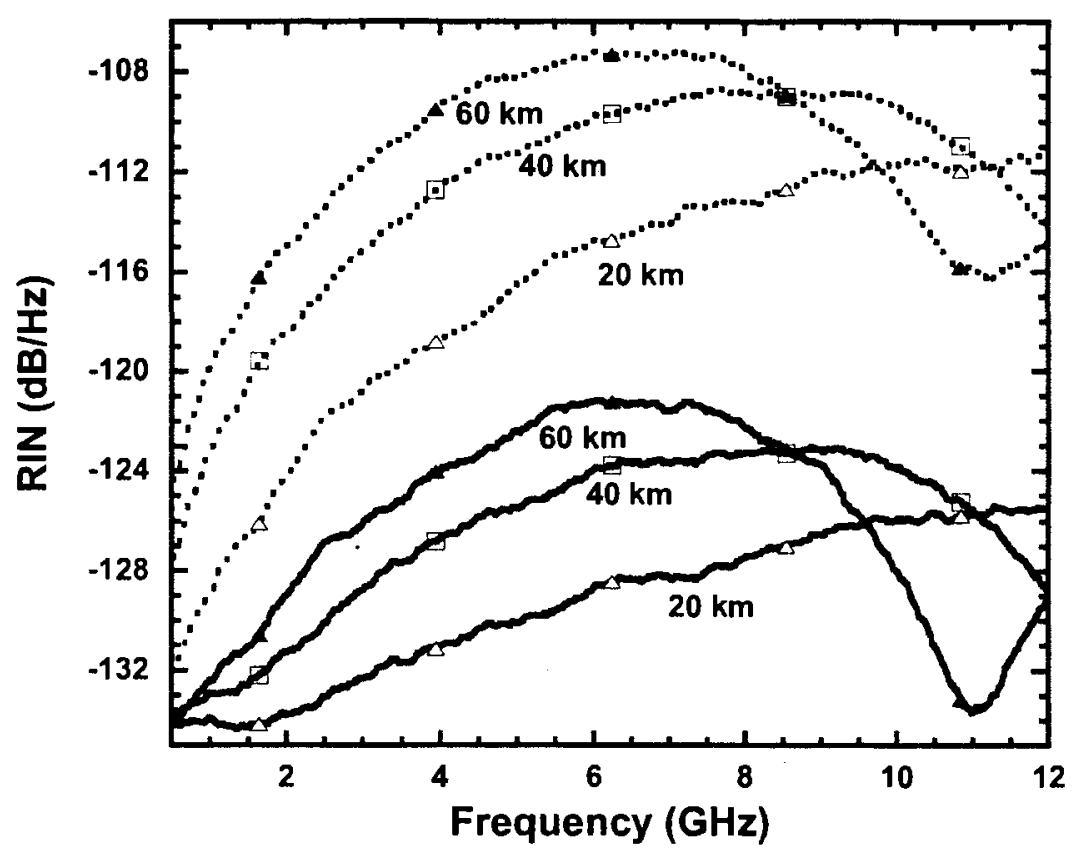

Figure 5.16 Calculated RIN versus frequency for 20,40 and $60 \mathrm{~km}$ (Solid: linewidth of $30 \mathrm{MHz}$. Dotted: linewidth of $1 \mathrm{GHz}$ ).

\subsubsection{Laser Linewidth, Fiber Dispersion and RF frequency}

It is well known that laser RIN can be enhanced by group velocity dispersion in optical fiber. In addition, previous work shows that laser RIN due to phase to intensity noise conversion by fiber dispersion is directly proportional to the laser linewidth [3031]. Calculated spectral density of RIN as a parameter of frequency for the laser source depicted in Fig. 4.1 is shown in Fig. 5.16 for two different linewidths of $30 \mathrm{MHz}$ and 1 $\mathrm{GHz}$ for fiber transmission of 20,40 and $60 \mathrm{~km}$, respectively. 


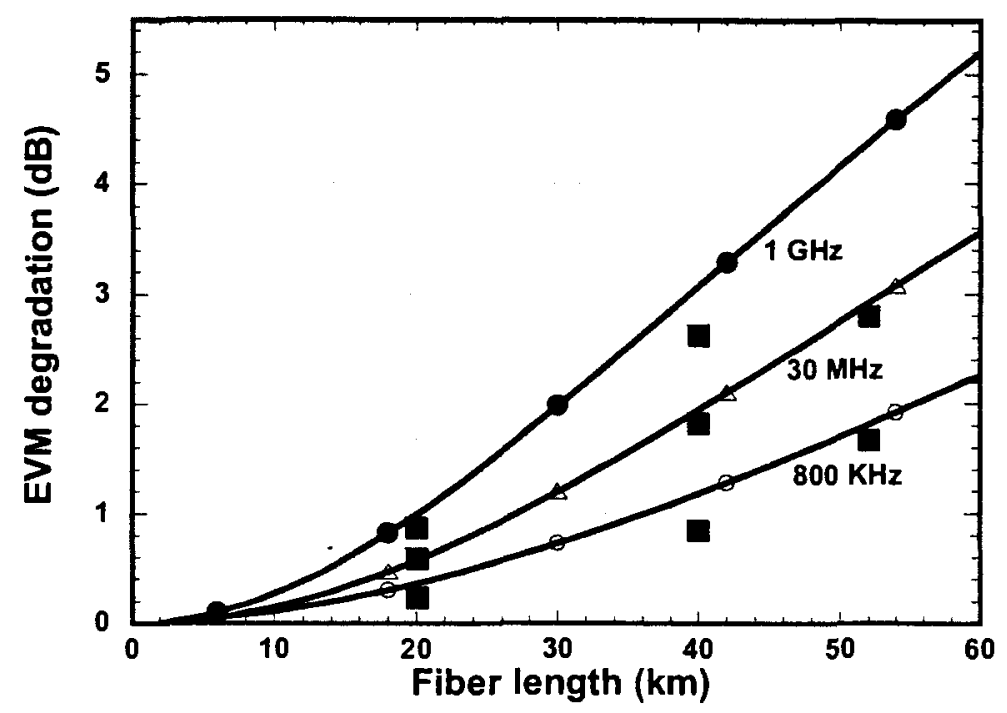

Figure 5.17 Calculated EVM degradation versus fiber length with respect to back-to-back. Square: experimental results for 20,40 and $52 \mathrm{~km}$.

To understand the effect of laser linewidth on RIN and EVM performance, we choose the second band centered at $3.96 \mathrm{GHz}$ and bandwidth of $528 \mathrm{MHz}$. Calculated EVM degradation of the system versus fiber length using Eq. (4.14) is shown in Fig. 5.17 for three different laser linewidths. The laser depicted in Fig. 4.1 has an intrinsic RIN of $145 \mathrm{~dB} / \mathrm{Hz}$ with linewidth of $30 \mathrm{MHz}$ and $1 \mathrm{GHz}$. The other laser has $800 \mathrm{kHz}$ linewidth and $-155 \mathrm{~dB} / \mathrm{Hz}$ intrinsic RIN. The experimental EVM is also shown in Fig. 5.17. Calculated results are in good agreement with the measurement in Fig. 5.17. However, the experimental EVM is slightly better because of the complex demodulation and error correction schemes used at the UWB receiver. Compared to the back to back, it is clearly shown that EVM degradation of $\sim 0.36$ and $\sim 1.1 \mathrm{~dB}$ is expected for $800 \mathrm{kHz}$ laser, whereas it is $\sim 0.58$ and $\sim 1.95 \mathrm{~dB}$ for $30 \mathrm{MHz}$ laser, after 20 and $40 \mathrm{~km}$ of fiber transmission, respectively. For the laser with linewidth of $1 \mathrm{GHz}$, the EVM degradation is $\sim 1$ and $\sim 3 \mathrm{~dB}$ for the two fiber lengths. The EVM degradation shown in Fig. 5.17 is due 
to increased RIN due to the interaction of the laser phase noise and chromatic dispersion as shown in Fig. 5.16.

At last one of the most important factors is the UWB carrier frequency of the UWB system. MB UWB has 14 bands with center frequency $f_{c}(M H z)=2904+528 n_{b}$, $n_{b}=1,2, \ldots, 14$. We perform simulation for all the 14 bands of MB OFDM using VPI TransmissionMaker software. Figure 5.18 shows simulated EVM for 14 bands UWB over fiber with fiber length of back-to-back, 20,40 and $60 \mathrm{~km}$, considering bit rate of 200 $\mathrm{Mb} / \mathrm{s}$ and laser linewidth of $30 \mathrm{MHz}$. The experimentally measured EVM is also shown in Fig. 5.18 for 20 and $40 \mathrm{~km}$ for bands centered at $3.432,3.96$ and $4.488 \mathrm{GHz}$.

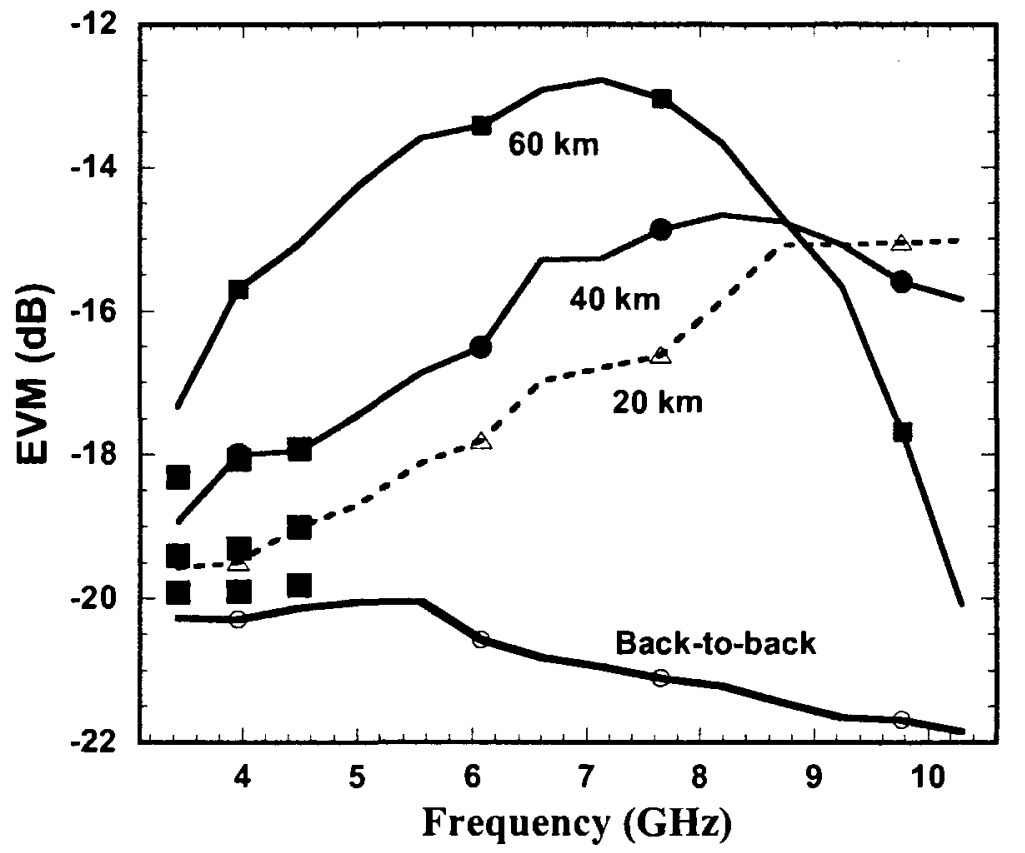

Figure 5.18 Simulated (line) EVM versus bands in an MB UWB over fiber system. Square: experimental results for 0,20 and $40 \mathrm{~km}$ centered at $3.432,3.96$ and $4.488 \mathrm{GHz}$ band. 
The results from Fig. 5.18 shows that for back to back as the frequency increases, EVM initially degrades up to the RIN peak, then it is improved after $5.544 \mathrm{GHz}$ band. However, with fiber dispersion the scenario is completely different. For $20 \mathrm{~km}$ of fiber, dispersion shifts the peak of RIN to upper frequency bands as shown in Fig. 5.16 and as the frequency increases the EVM also increases for all the frequency bands. Also for 20 $\mathrm{km}$ of fiber, from Fig. 5.16 we see the RIN becomes almost constant after $9.768 \mathrm{GHz}$ band and so does the EVM. With fiber transmission link of $40 \mathrm{~km}$ the EVM initially increases until $8.184 \mathrm{GHz}$ band than gradually decreases as the RIN drops in value. The result for $60 \mathrm{~km}$ fiber transmission link is quite interesting. Due to sinusoidal behavior of the dispersion induced phase noise to intensity noise conversion the RIN begins to decrease considerably after $7.656 \mathrm{GHz}$ band and EVM is improved rapidly.

\subsection{Performance of Multi-band OFDM Ultra-Wideband over} Fiber Transmission under the Presence of In Band Interferers

For real world applications UWB receivers face jamming scenarios from multiple in-band and out of band interferers. Some of the typical interferers at the input of a MB OFDM receiver are shown in Fig. 1. IEEE $802.11 \mathrm{~b} / \mathrm{g}$, Bluetooth and microwave ovens act as out of band interferers. WiMAX at $3.5 \mathrm{GHz}$ interferes with band group 1, IEEE 
$802.11 \mathrm{a} / \mathrm{n}$ interferes with band group 2 and radar signal interferes with band group 4 , respectively.

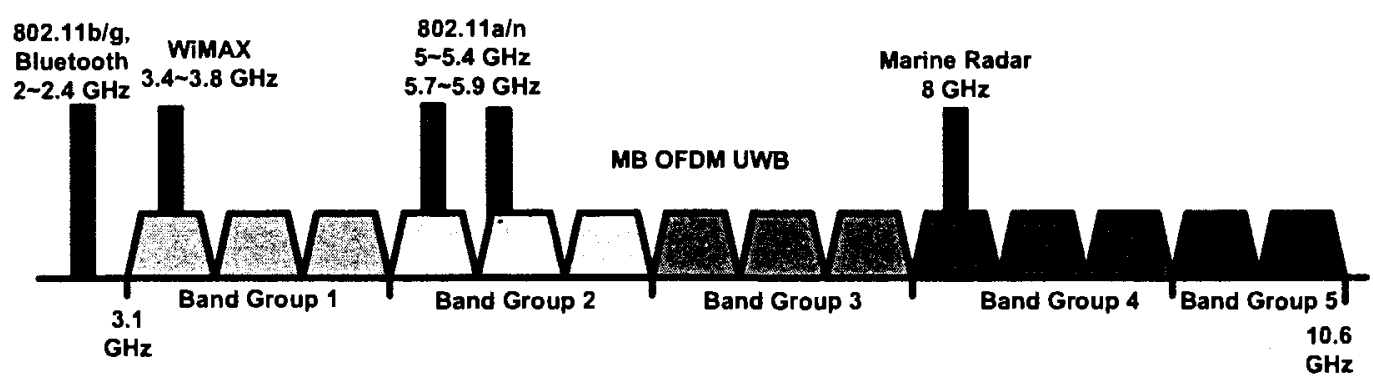

Figure 5.19 Spectrum of UWB signal with narrow band interferes.

In this section we analyze EVM performance of MB UWB over fiber under different in band interference scenarios. We first investigate the system performance without any in-band interference and use the information to compare degradation under interference conditions. To emulate $1 \mathrm{~m}$ transmission over air under real antenna scenarios we added two multipath version of our generated signal with delay of 1 symbol and 2 symbols, relative amplitude of $-35 \mathrm{~dB}$ and $-40 \mathrm{~dB}$ and phase of 5 and 10 degree, respectively from the arbitrary waveform generator before transmission through the fiber. We tried to make our results independent of the amplification at the receiver front end so we present the results as a parameter of interferer to UWB peak power ratio rather than the power of interferer. For example free space path loss for $1 \mathrm{~m}$ can be emulated by inserting a $40 \mathrm{~dB}$ attenuator at point $\mathrm{A}$ in Fig. 4.3. In that case, extra RF amplification has to be provided at point $\mathrm{B}$ in Fig. 4.3 by using a RF amplifier with higher gain and keep 
the RF modulation index at the electro optic modulator constant. It should be noted that EVM is the ratio of the distortion in the received constellation with respect to transmitted constellation. So, theoretically fixed gain or attenuation at the front end is a common multiplication factor both for the numerator and denominator and does not have any impact on EVM performance.

\subsubsection{UWB over Single Mode Fiber without Interferers}

We turn off the interferers from arbitrary waveform generator and transmit only MB UWB signal of band group 1, band group 2 and band group 4 according to WiMedia specification as described in Table 4.1 through the fiber one by one and measure the EVM at point $F$ in Fig. 4.3.

We also measure the intrinsic EVM of the generator, where the output of generator at point $\mathrm{A}$ is directly connected to point $\mathrm{F}$ in Fig. 4.3 using a coaxial cable. Results from our measurement are enlisted in Table 5.1. The results show that EVM degradation due to electrical to optical conversion is usually $2 \sim 3 \mathrm{~dB}$. EVM performance for back-to-back (B-B), i.e. no actual fiber transmission is around $-24 \mathrm{~dB}$ for all the band groups. This value increases by $1.4 \sim 2 \mathrm{~dB}$ for $20 \mathrm{~km}$ fiber transmission. At high frequencies there is a higher degradation in EVM performance for $20 \mathrm{~km}$ fiber transmission compared to back-to-back due to fiber chromatic dispersion induced laser phase to intensity noise conversion or relative intensity noise [30]. The results obtained are still much better than the WiMedia defined -16 dB limit of EVM [4] and for lower band groups and the fiber link can be easily extended up to $60 \sim 80 \mathrm{~km}$ of single mode 
fiber depending on the quality of the devices used for electrical to optical and optical to electrical conversion.

Table 5.1 Measured EVM Performance of UWB Transmission without Interferer.

\begin{tabular}{|l|c|c|c|}
\hline Band Group & Intrinsic & B-B & 20 km of SMF \\
\hline Band group 1 & $-27.32 \mathrm{~dB}$ & $-24.50 \mathrm{~dB}$ & $-23.12 \mathrm{~dB}$ \\
\hline Band group 2 & $-26.21 \mathrm{~dB}$ & $-24.25 \mathrm{~dB}$ & $-22.65 \mathrm{~dB}$ \\
\hline Band group 4 & $-25.52 \mathrm{~dB}$ & $-23.44 \mathrm{~dB}$ & $-21.30 \mathrm{~dB}$ \\
\hline
\end{tabular}

\subsubsection{Performance of Band Group 1 of MB OFDM UWB under the presence of WiMAX with Fiber Distribution}

Recently (21/5/2008) European Commission opened the radio spectrum 3.4 3.8 GHz for WiMAX applications [12]. This notion raised many concerns among the UWB communities because WiMAX will interefere with band group 1. Band goup 1 of UWB was thought to have the most potential because of mature CMOS technology in this frequency range and absence of other interferers. Subsequently, how WiMAX will affect UWB over fiber transmission has become a cause of apprehension among researchers. We study the performance of UWB over fiber transmission under the presence of WiMAX. We generate a $20 \mathrm{MHz}$ wide WiMAX signal centered at $3.5 \mathrm{GHz}$ with 64 Qudrature Amplitude Modulation (QAM) and a bit rate of $54 \mathrm{Mb} / \mathrm{s}$ based on IEEE 
802.16-2004 technology [33]. The signal has 256 OFDM subcarriers among which 56 are null and guard tones, 8 are pilots and the rest 192 are data tones.

Transmitted RF spectrum of UWB and WiMAX at point A and received spectrum after $20 \mathrm{~km}$ at point $\mathrm{F}$ in Fig. 4.3 is showed in Fig. 5.20(a) and (b), respectively. The results from EVM measurement presented in Fig. 5.21 shows that EVM performance of UWB over fiber transmission is severely degraded under presence of WiMAX. If the WiMAX to UWB peak power ratio is more than $\sim 14 \mathrm{~dB}$ the transmission performance quickly falls below WiMedia defined limit of $-16 \mathrm{~dB}$. The performance degradation is mainly due to large number of subcarriers presents in WiMAX within a small bandwidth so the sub-carriers interact with each other and the OFDM subcarriers in MB OFDM UWB signal resulting in a high carrier leakage and inter carrier interference.

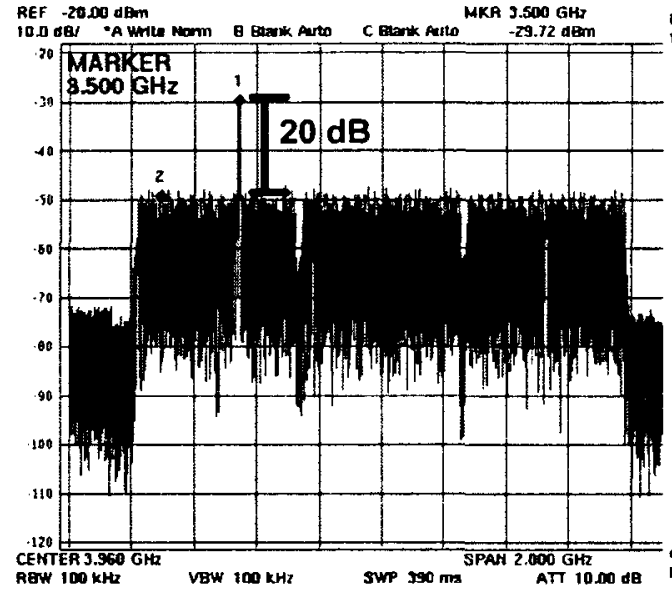

(a)

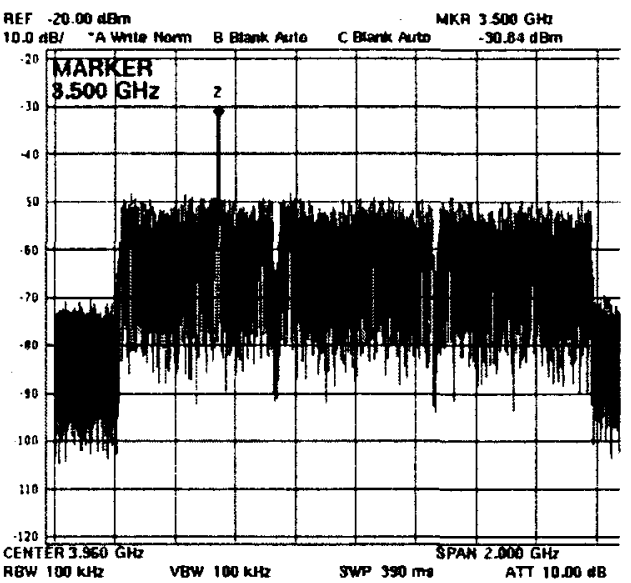

(b)

Figure 5.20 RF spectrum of UWB band group 1 and WiMAX (a) transmitted at point A (b) received at point $F$ in Fig. 2 for bit rate of $200 \mathrm{Mb} / \mathrm{s}$ with $20 \mathrm{~km}$ fiber transmission (Interferer to UWB peak power ratio is $20 \mathrm{~dB}$ ). 


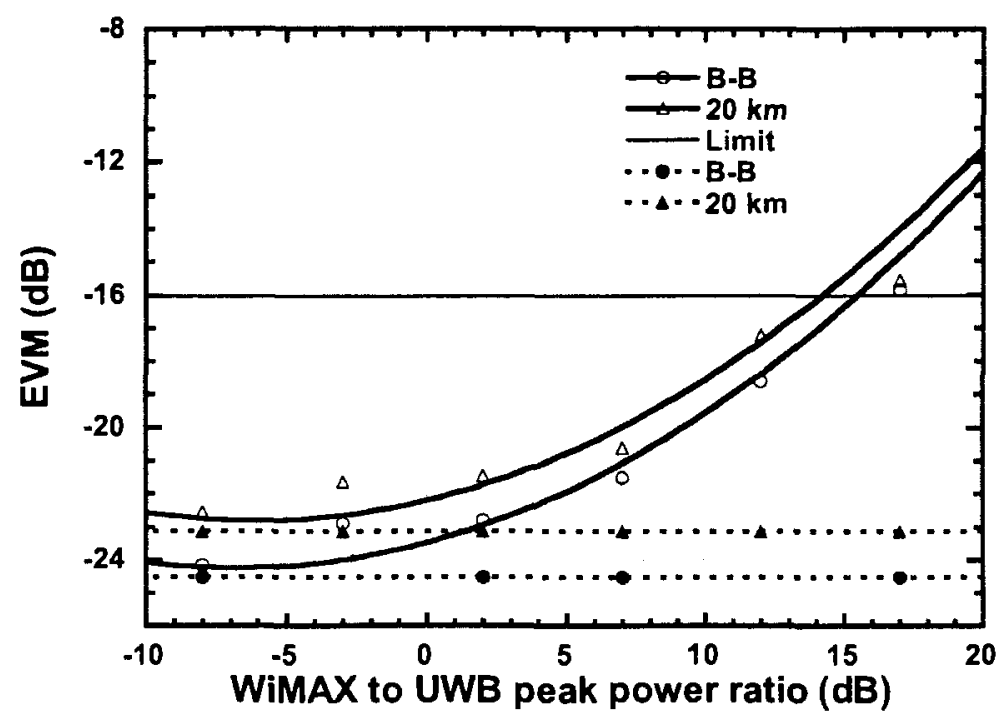

Figure 5.21 EVM performance of UWB over fiber transmission under the presence of WiMAX as a function of WiMAX to UWB peak power ratio (Solid lines: best fitted curves, dotted lines: without interference).

\subsubsection{Performance of Band Group 2 of MB OFDM UWB under the presence of WLAN MIMO and WLAN with Fiber Distribution}

IEEE $802.11 \mathrm{n}$ MIMO is an emerging technology that can provide throughput higher than $100 \mathrm{Mb} / \mathrm{s}$ for access network applications. The standard is currently in final stage and it is expected it will be ratified in November 2009 [34]. So interference from MIMO to UWB is an interesting research topic. We generate a $54 \mathrm{Mb} / \mathrm{s}$ WLAN MIMO signal according to IEEE $802.1 \mathrm{ln}$ standard. The signal uses OFDM modulation of 64 subcarriers each modulated with 64 QAM. The signal also uses space time block coding (STBC) which makes the power distribution and spectral density in the RF signal different from conventional WLAN. Transmitted RF spectrum of UWB and WLAN 
MIMO at point $A$ and received spectrum after $20 \mathrm{~km}$ at point $F$ in Fig. 4.3 is showed in Fig. 5.22(a) and (b), respectively.

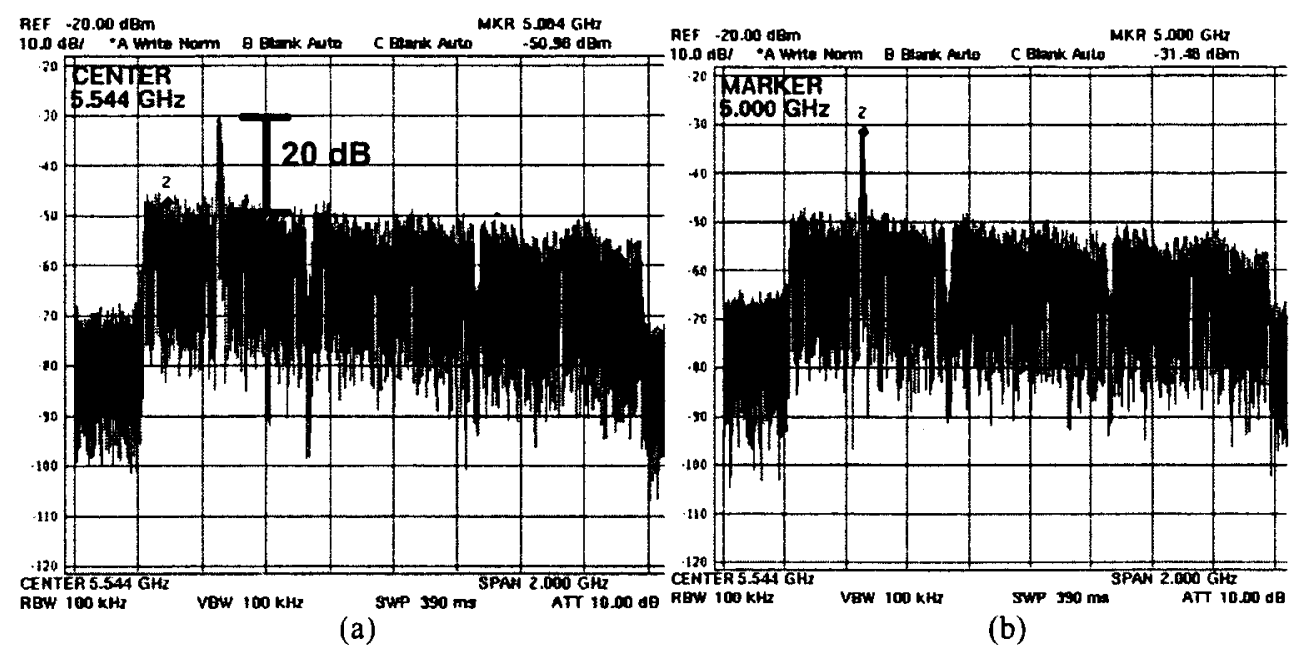

Figure 5.22 RF spectrum of UWB band group 1 and WLAN MIMO (a) transmitted at point A (b) received at point $\mathrm{F}$ in Fig. 2 for bit rate of $200 \mathrm{Mb} / \mathrm{s}$ with $20 \mathrm{~km}$ fiber transmission (Interferer to UWB peak power ratio is $20 \mathrm{~dB}$ ).

Measured EVM is shown in Fig. 5.23 and it is found that EVM of the transmitted UWB signal degrades as the WLAN MIMO's power is increased but acceptable performance is found if the WLAN MIMO's peak power level is not higher than UWB peak power level by more than $\sim 17.5 \mathrm{~dB}$. 


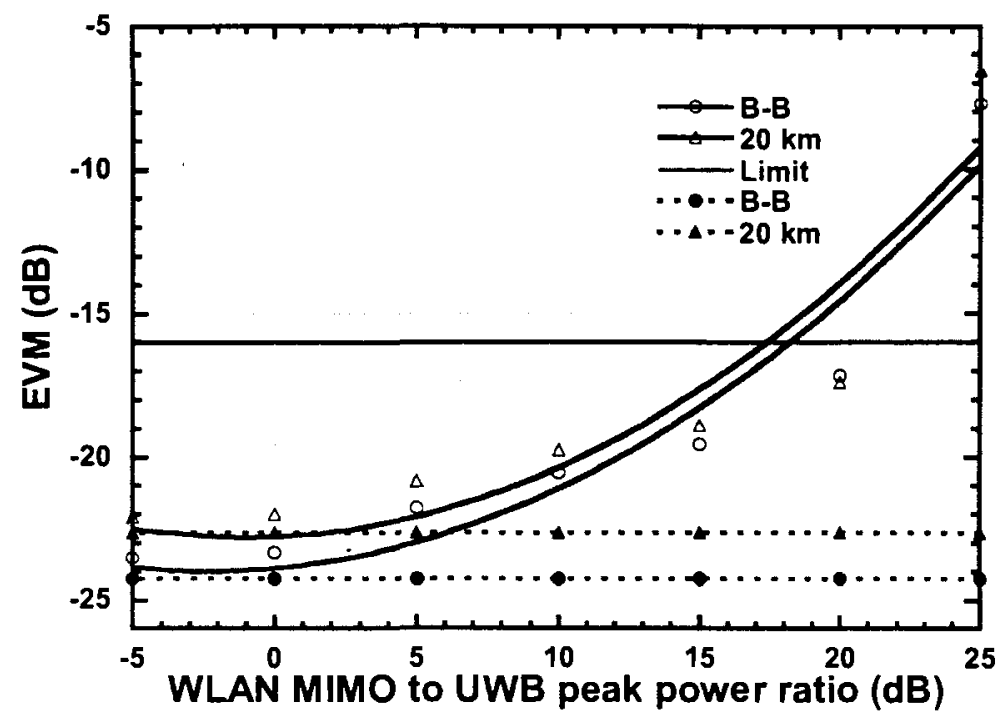

Figure 5.23 EVM performance of UWB over fiber transmission under the presence of WLAN MIMO as a function of WLAN MIMO to UWB peak power ratio (Solid lines: best fitted curves, dotted lines: without interference).

IEEE 802.11a, conventional WLAN is a widely used technology. WLAN signal consists of a $20 \mathrm{MHz}$ channel with center frequency $5.8 \mathrm{GHz}$ comprising of 64 subcarriers of which 52 are effective subcarriers, 48 carrying data and 4 pilots [36]. The modulation scheme is 64 QAM OFDM with bit rate of $54 \mathrm{Mb} / \mathrm{s}$. Transmitted RF spectrum of UWB and WLAN at point $A$ and received spectrum after $20 \mathrm{~km}$ at point $\mathrm{F}$ in Fig. 4.3 is showed in Fig. 5.24(a) and (b), respectively. Our experimental results from EVM measurement of UWB over fiber transmission is shown in Fig 5.25. The EVM is almost constant for back-to-back and $20 \mathrm{~km}$ fiber transmission until WLAN to MB UWB peak power level ratio reaches $10 \mathrm{~dB}$. After that the EVM begins to decrease slowly in 
both cases. The EVM falls below $-16 \mathrm{~dB}$ limit if the peak power ratio is more than $20 \mathrm{~dB}$ and $21 \mathrm{~dB}$, respectively for back-to-back and $20 \mathrm{~km}$ fiber distribution.

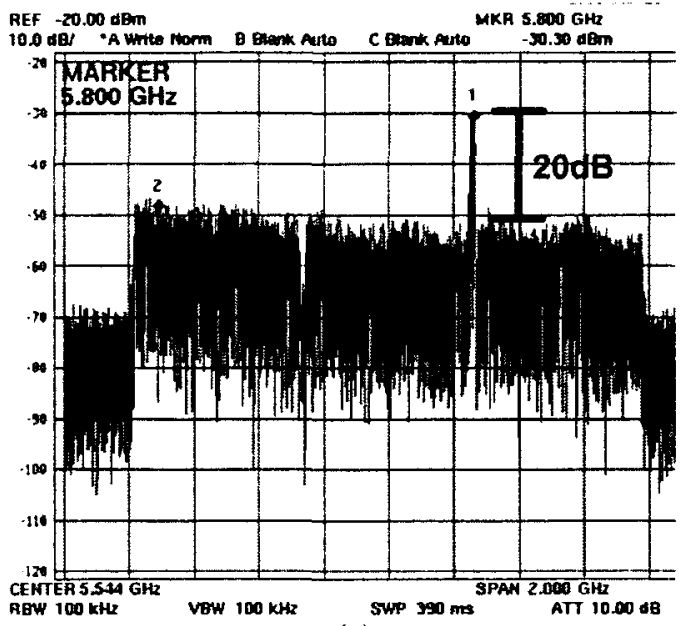

(a)

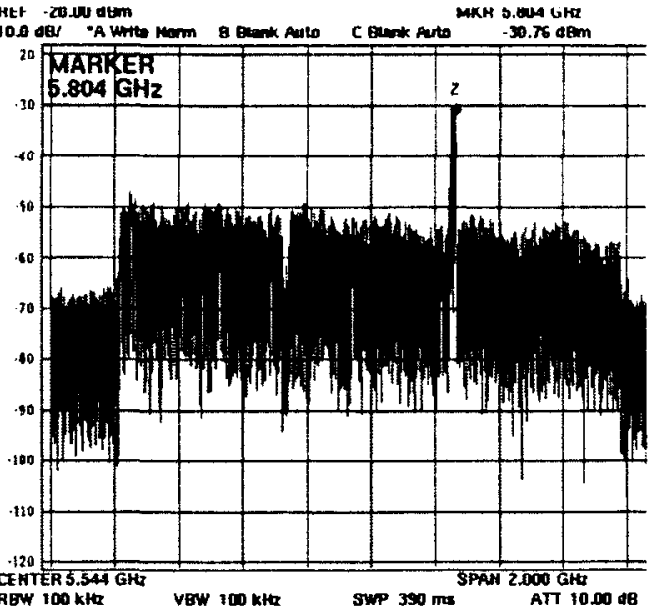

(b)

Figure 5.24 RF spectrum of UWB band group 2 and WLAN (a) transmitted at point A (b) received at point $\mathrm{F}$ in Fig. 2. for bit rate of $200 \mathrm{Mb} / \mathrm{s}$ with $20 \mathrm{~km}$ fiber transmission (Interferer to UWB peak power ratio is $20 \mathrm{~dB}$ ).

It should be noted that WLAN signals interfere with UWB signals much less than WiMAX due to the fact that the number of subcarriers carrying data in WLAN is only one fourth of that in WiMAX. As a result, WLAN will disrupt smaller numbers of subcarriers in UWB when compared to WiMAX. 


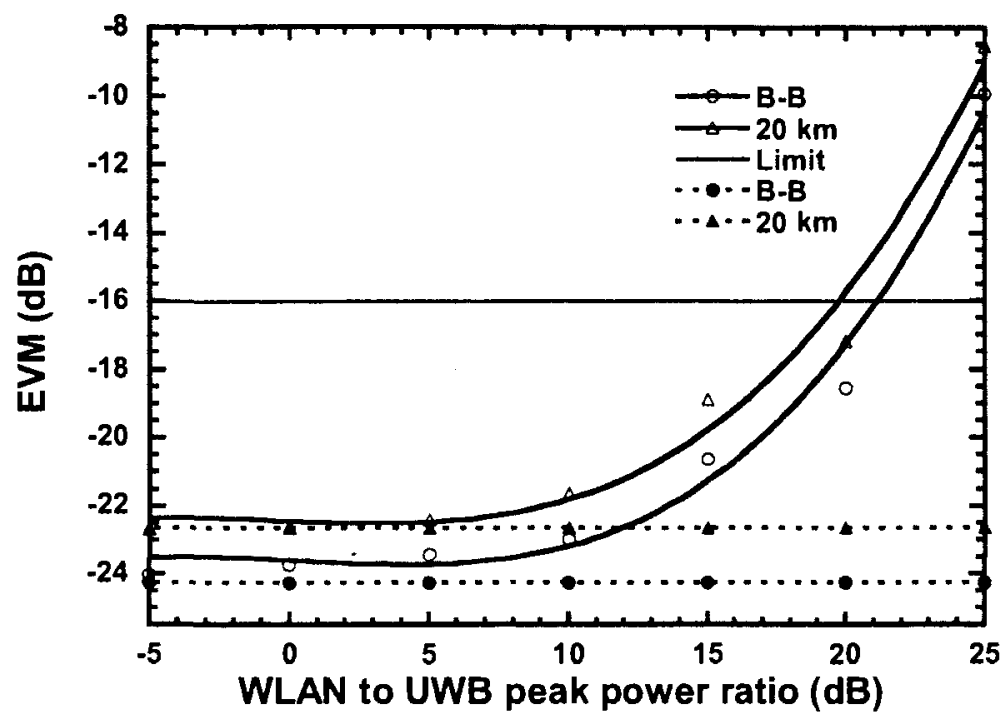

Figure 5.25 EVM performance of UWB over fiber transmission under the presence of WLAN as a function of WLAN to UWB peak power ratio (Solid lines: best fitted curves, dotted lines: without interference).

\subsubsection{Performance of Band Group 4 of MB OFDM UWB under the} presence of Marine Radar with Fiber Distribution

Radar signals at high frequency can also interfere with MB UWB signals. We used a linear frequency modulated pulse (LFM) centered at $8 \mathrm{GHz}$ with pulse width of 5 $\mu$ s and frequency sweep of $20 \mathrm{MHz}$ as a radar source and integrate with band group 4 of UWB signal. Transmitted RF spectrum of UWB and marine radar and received spectrum after $20 \mathrm{~km}$ is showed in Fig. 5.26(a) and (b), respectively. 


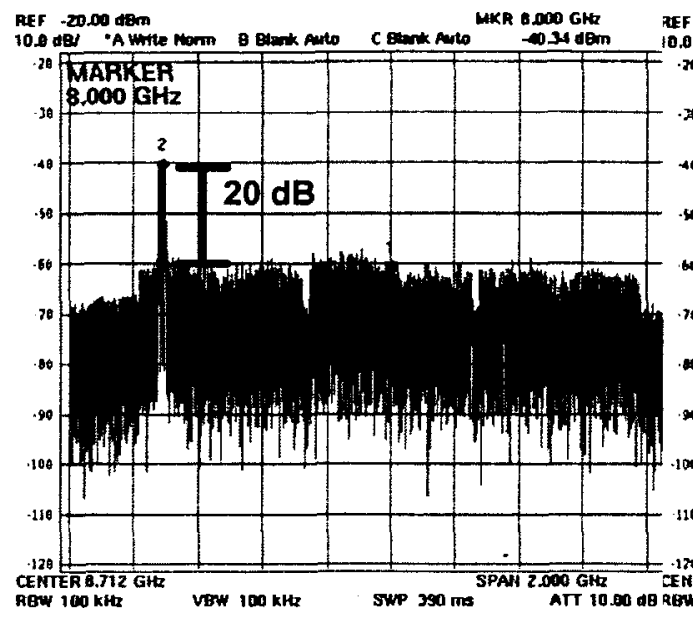

(a)

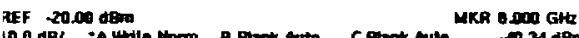

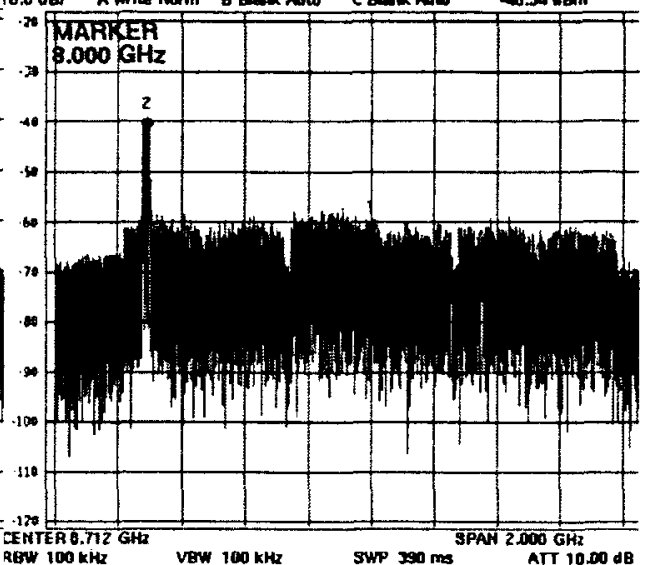

(b)

Figure 5.26 RF spectrum of UWB band group 4 and marine radar (a) transmitted at point A (b) received at point $\mathrm{F}$ in Fig. 2 for bit rate of $200 \mathrm{Mb} / \mathrm{s}$ with $20 \mathrm{~km}$ fiber transmission (Interferer to UWB peak power ratio is $20 \mathrm{~dB}$ ).

The EVM performance of UWB as a parameter of radar peak power to UWB peak power is shown in Fig. 5.27. The EVM degrades quickly if the radar peak power is increased for both back-to-back and $20 \mathrm{~km}$ fiber transmission. Referring to Fig. 5.27, EVM value of $-16 \mathrm{~dB}$ is found for radar to UWB peak power ratio of $17 \mathrm{~dB}$ and $15 \mathrm{~dB}$ for back-to-back and $20 \mathrm{~km}$ fiber transmission, respectively. Large difference between back-to-back and $20 \mathrm{~km}$ fiber distribution is due to laser relative intensity noise as explained in Sub-section A. Experiments with $\mathrm{C}$ band military radar in the frequency range of 5.250 5.925 GHz and band group $2(4.752 \sim 6.336 \mathrm{GHz})$ give similar results. 


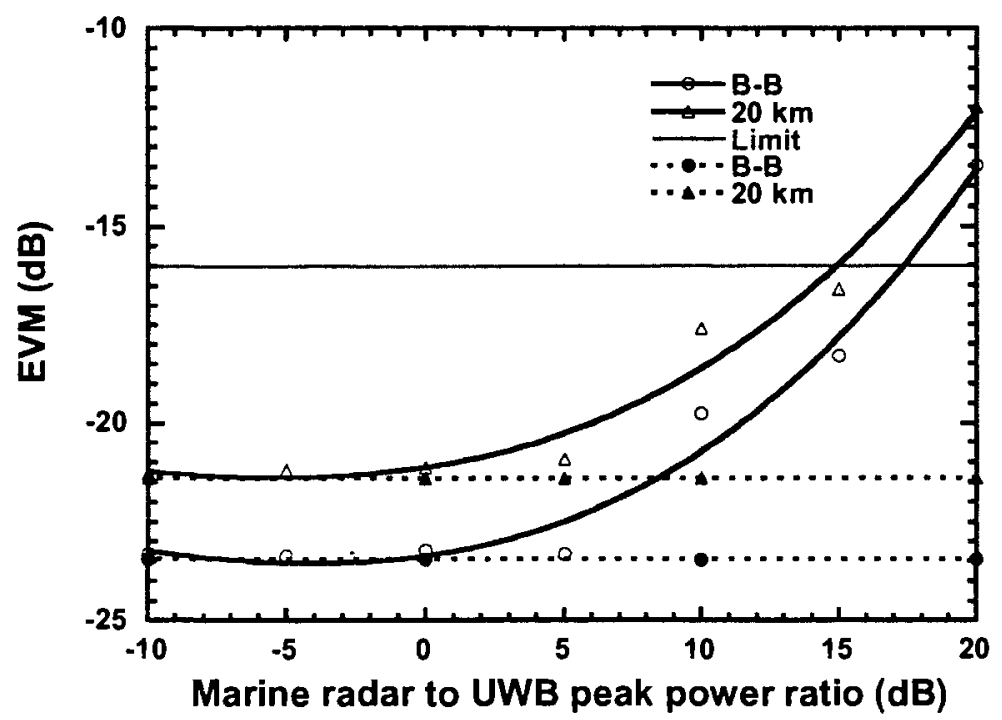

Figure 5.27 EVM performance of UWB over fiber transmission under the presence of WLAN as a function of WLAN to UWB peak power ratio (Solid lines: best fitted curves, dotted lines: without interference).

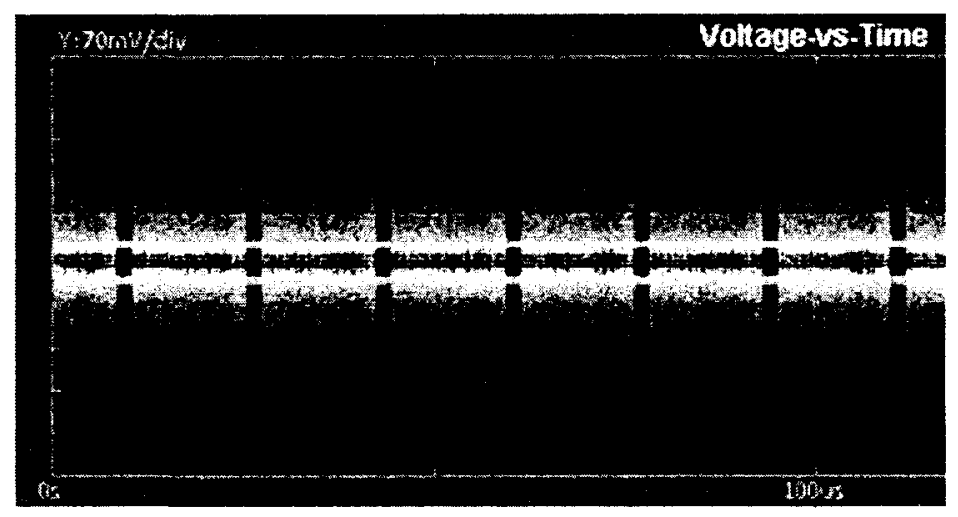

Figure 5.28 Received time domain spectrum for band group 4 after $20 \mathrm{Km}$ of fiber transmission with bit rate of $200 \mathrm{Mb} / \mathrm{s}$ without any signal interferer. 


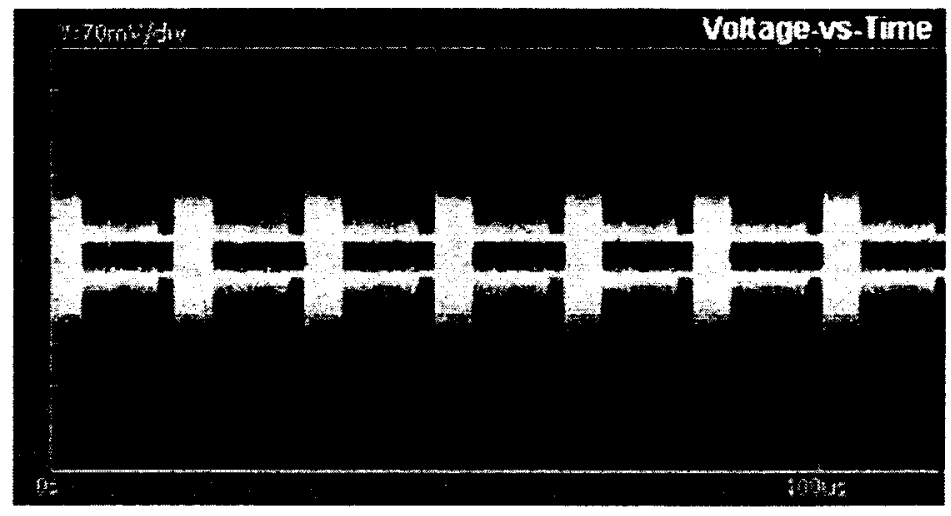

Figure 5.29 Received time domain spectrum for band group 4 after $20 \mathrm{Km}$ of fiber transmission with bit rate of $200 \mathrm{Mb} / \mathrm{s}$ with radar signal as interferer (Interferer to UWB peak power ratio is 20 $\mathrm{dB})$.

Low duty cycle pulses from radar signal interact and disrupt the subcarriers in UWB signal. Time domain spectrum after $20 \mathrm{~km}$ of fiber transmission without and with radar signal as interferer is presented in Fig. 5.28 and 5.29 , respectively. It is clearly visible that radar signal distorts the waveform at the edges. It should be noted that in UWB receivers most of the signal processing is performed in time domain. Therefore, any short pulse like radar in time domain jams the UWB signal at UWB receiver input and causes the degradation of EVM. 


\section{Chapter 6 Conclusions}

\subsection{Summary}

We have experimentally investigated and theoretically analyzed the performance of MB-OFDM UWB when transmitted over fiber. EVM is employed to evaluate the system quality of UWB signal considering system's parameters such as RF modulation index of MZM, fiber transmission and received optical power. Performance degradation of MB-OFDM UWB caused by various impairments including MZM nonlinearities, fiber dispersion and optical receiver response are also comprehensively investigated.

It is found that for RF modulation index of up to $\sim 4 \%$ OFDM subcarriers suffer from relative phase shift due to fiber dispersion and are immune to amplitude distortion that could be induced by the combined effect of MZM response nonlinearities and fiber dispersion. However, if modulation index is more than $4 \%$ the OFDM subcarriers suffer from both amplitude and phase distortion due to the combined effect of MZM response nonlinearities and fiber dispersion. Therefore modulation index of $4 \%$ is optimum to achieve the best EVM.

Moreover, when the optimum modulation index is used fiber transmission is further limited by laser phase noise converted RIN due to fiber dispersion and phase distortion induced by fiber dispersion in addition to increase of optical amplifier noise due to fiber loss, compared to back to back UWB over fiber.

Also, it has been found that the optical receiver response has significant impact on EVM performance. It is found that Chebyshev-II response with $3 \mathrm{GHz}$ and fifth order 
is the best for MB OFDM UWB over fiber. This is due to low amplitude and phase distortion within the passband. Since the UWB over fiber is operated at a low RF modulation index and multiband UWB has a low power spectral density, it is found that required optical power at optical receiver in UWB over fiber is reasonably higher than that in radio over fiber with other modulation schemes. Furthermore, we have found that EVM in UWB over fiber is degraded almost linearly with the decrease of receiver optical power.

The performance of multi-band OFDM UWB was investigated when transmitted over fiber under the effect of relative intensity noise considering system's parameters such as laser output power, linewidth and fiber transmission length. It is found that the system should be operated at a high laser output power to avoid RIN degradation. It is shown using a narrow linewidth laser with low RIN will significantly improve system performance. Also simulations were performed for all the 14 bands of MB UWB to show performance dependence of RF carrier frequency. The results show bands higher than 7.656 $\mathrm{GHz}$ are critically affected depending on fiber dispersion induced phase to intensity noise conversion.

It is found different in-band interferers can affect the performance of MB-OFDM UWB severely if certain interferer to UWB peak power ratio is not maintained. For WiMax/marine radar and UWB devices that are co-located this ratio is only $\sim 14$ and $\sim 15$ $\mathrm{dB}$, respectively for WiMAX and radar signals if distributed over $20 \mathrm{~km}$ of single mode fiber. MB OFDM UWB over fiber is more resistant to interference from WLAN MIMO and conventional WLAN. The interferer to MB OFDM UWB peak power ratio is $\sim 17.5$ and $\sim 20 \mathrm{~dB}$ for $20 \mathrm{~km}$ fiber transmission for WLAN MIMO and conventional WLAN, 
respectively for satisfying the EVM requirement of $-16 \mathrm{~dB}$, which is the EVM threshold specified for conformance testing according to the recent WiMedia standard for UWB. Our results will allow the future researchers in the field of MB OFDM UWB over fiber transmission to optimize performance UWB over fiber transmission under the presence of all possible in-band interferers.

\subsection{Future Works}

MB-OFDM UWB over fiber is a fast emerging technology. However, many areas of UWB over fiber are yet to be explored.

First, the basic form of UWB over fiber uses a single optical channel. However, initial cost of laying fiber in an access network is high and with single channel system only a fraction of its enormous bandwidth is utilized. Optical wavelength division multiplexing (WDM) has several advantages- 1) Effective use of fiber bandwidth, i.e. up to 64 channels can be supported. With time and frequency interleaving properties of $\mathrm{MB}$ UWB hundreds of subscribers can be supported with single fiber. This can be very handy in wireless hotspots where service has to be provided to large numbers of subscribers. 2) WDM based on arrayed waveguide grating (AWG) is highly scalable and favourable to future network extension. 3) In WDM, different optical line terminal (OLT) and optical network units (ONUs) can support different bit rates. This is very important for MB

UWB because transmission distance in UWB is constrained by bit rate i.e. $53.3 \mathrm{Mb} / \mathrm{s}$ covers $10 \mathrm{~m}$ and $480 \mathrm{Mb} / \mathrm{s}$ covers only $4 \mathrm{~m} .4$ ) Using different optical carriers provide 
physical separation between subcarrier signals. 5) WDM is colorless. It supports any type of internet protocol (IP), video and data services. Especially in the case of video surveillance system for long tunnels WDM UWB can be an economical way to transfer simultaneously a number of video streams and control signals. As a result, MB-OFDM UWB over WDM is an open research challenge.

Bi-directional transmission over fiber is another important issue that needs to be addressed. Bi-directional MB-OFDM UWB has great prospect for future access network applications. If same optical wavelength is used, the study of stimulated Raman scattering (SRS) and stimulated brillouin scattering (SBS) will be an interesting research topic.

Third, investigation of performance of optical transmission by using a low cost Electro-absorption modulator (EAM) or integrated DFB laser-EAM (EML) can be done.

Also, the effect of cross interference from narrowband jammers is not studied in this thesis. Practically two in-band jammers can exist simultaneously i.e. IEEE $802.11 \mathrm{~b} / \mathrm{g}$ at $2.4 \mathrm{GHz}$ and $802.11 \mathrm{a}$ at $5.8 \mathrm{GHz}$ can beat together to create a harmonics at $3.4 \mathrm{GHz}$ which will interfere with sub-band 1 Band Group 1.

Besides, MB-OFDM UWB over fiber technology will benefit from studies on MAC layer protocols for optimum transmission performance. 


\section{References}

[1] Federal Communications Commission, Revision of part 15 of the Commission's rules regarding ultra-wideband transmission system first report and order, ET-Docket 98153, FCC02-48, Feb. 2002.

[2] "Multi-band OFDM physical layer proposal for IEEE 802.15 Task Group 3a", IEEE P802.15 Working Group for WPAN, Mar. 2004.

[3] G. Heidari, WiMedia UWB: technology of choice for wireless USB and Bluetooth, Chapter 1, lst ed. Wiley, 2008.

[4] ECMA-368, High rate ultra wideband PHY and MAC standard, ECMA International, Geneva, 2nd edition, Dec. 2007.

[5] Y. Guennec, M. Lourdiane, B. Cabon, G. Maury, P. Lombard, "Technologies for UWB-over fiber," in Proc. IEEE Lasers and Electro-Optics Society (LEOS) Annual Meeting, pp. 518-519, Oct. 2006.

[6] A. Pizzinat, B. Charbonnier, M. Moignard, "Analysis of laser induced distortions in ultra wide band MB-OFDM over fiber," in Proc. IEEE Lasers and Electro-Optics Society (LEOS) Annual Meeting, pp. 339-340, Oct. 2006.

[7] M. Yee, V. Pham, Y. Guo, L. Ong, B. Luo, "Performance evaluation of MB-OFDM ultra-wideband signals over single mode fiber," in Proc. International Conference on the Ultra Wideband (ICUWB), pp. 674-677, Sep. 2007. 
[8] A. Pizzinat, P. Urvoas, B. Charbonnier, "1.92 Gbit/s MB-OFDM ultra wideband radio transmission over low bandwidth multimode fiber," in Proc. Optical Fiber Communication Conference and Exposition (OFC) and The National Fiber Optic Engineers Conference (NFOEC), pp. 1-3, Feb. 2007.

[9] Y. Guo, V. Pham, M. Yee, L. Ong, B. Luo, "Performance study of MB-OFDM ultrawideband signals over multimode fiber," in Proc. International Conference on the Ultra Wideband (ICUWB), pp. 429-431, Sept. 2007.

[10] Y. Ben-Ezra, M. Ran, E. Borohovich, A. Leibovich, M. Thakur, R. Llorente, S. Walker, "Wimedia-Defined, ultra-wideband radio transmission over optical fibre," in Proc. Optical Fiber Communication Conference and Exposition (OFC) and The National Fiber Optic Engineers Conference (NFOEC), pp. 1-3, Feb. 2008.

[11] M. Yee, V. Pham, Y. Guo, L. Ong, B. Luo, "Performance evaluation of multiband radio-over fiber for WLAN, Gigabit Ethernet and UWB," in Proc. IEEE International Microwave Symposium (IMS), pp. 491-494, Jun. 2008.

[12] R. Llorente, T. Alves, M. Morant, M. Beltran, J. Perez, A. Cartaxo, J. Marti, "Optical distribution of OFDM and impulse-Radio UWB in FTTH networks," in Proc. Optical Fiber Communication Conference and Exposition (OFC) and The National Fiber Optic Engineers Conference (NFOEC), pp. 1-3, Feb. 2008.

[13] M. Jazayerifar, B. Cabon, J. Salehi, "Transmission of multi-band OFDM and impulse radio ultra- wideband signals over single mode fiber," J. Lightw. Technol., vol. 26 , no. 15 , pp. 2594-2603, Aug. 2008. 
[14] D. Smith, A. Borghesani, D. Moodie, M. Thakur, T. Quinian, S. Dudley, M. Toycan, C. Bock, S. Walker, M. Ran, Y. Ben-Ezra, “480 Mbps ultra-wideband radio over fibre transmission using a $1310 / 1550 \mathrm{~nm}$ reflective electro-absorption transducer and offthe-shelf components," in Proc. Optical Fiber Communication Conference and Exposition (OFC) and The National Fiber Optic Engineers Conference (NFOEC), pp. 1-3, Feb. 2008.

[15] A. Pizzinat, F. Payoux, B. Charbonnier, S. Meyer, "Ultra wideband over fibre transparent architecture for high-bit-rate home networks," SpringerLink Annals of Telecommunications, vol. 63, no. 9-10, pp. 455-462, Oct. 2008.

[16] H. Al-Raweshidy, S. Komaki, Radio over fiber technologies for mobile communication networks, Chapter 1 to 5, 1st ed. Boston: Artech House, 2002.

[17] D. Linh, I. Niemegeers, "Analysis of IEEE 802.11 in Radio over Fiber Home Networks," in Proc. IEEE Conference on Local Computer Networks, pp. 744-747, Nov. 2005.

[18] P. Tang, L. Ong, A. Alphones, B. Luo, M. Fujise, "PER and EVM measurements of a radio-over-fiber network for cellular and WLAN system applications," J. Lightw. Technol., vol. 22, no. 11, pp. 2370-2376, Nov. 2004.

[19] T. Barrett, "History of ultra wideband communications and radar: part I, UWB communications," Microwave Journal, Feb. 2001.

[20] T. Barrett, "History of ultra wideband communications and radar: part II, UWB radars and sensors," Microwave Journal, Feb. 2001. 
[21] http://www.wimedia.org/en/resources/worldwide_regulatory.asp

[22] M. Welborn, "System considerations for ultra-wideband wireless networks," in Proc. IEEE Radio Wireless Conference, pp. 5-8, Aug. 2001.

[23] W. Siriwongpairat, K. Liu, Ultra-Wideband Communications Systems: Multiband OFDM Approach, 1st ed. IEEE press and Wiley \& Sons,Inc., 2008.

[24] S. Ghassemzadeh, V. Tarokh, "UWB Path Loss Characterization in Residential Environments," in Proc. Radio Freq. Integrated Circuits Symp., 2003, pp. 501-504.

[25] C. Zhao, R. Baxley, "Error vector magnitude analysis for OFDM systems," in Proc. Asilomar Conference on Signals, Systems, and Computers (ACSSC), pp. $1830-1834$, Oct.-Nov. 2006.

[26] A. Georgiadis, "Gain, phase imbalance, and phase noise effects on error vector magnitude," IEEE Trans. on Vehicular Tech, vol. 53, no. 2, pp. 443-449, Mar. 2004.

[27] M. Faulkner, "The effect of filtering on the performance of OFDM systems," IEEE Trans. on Vehicular Tech, vol. 49, no. 5, pp. 1877-1884, Sept. 2000.

[28] L. Paarmann, Design and analysis of analog filters, Chapter 5, Kluwer Academic Publishers, 2001.

[29] D. Pozar, Microwave Engineering, Chapter 8, 3rd ed. Wiley, 2007.

[30] W. Marshall, B. Crosignani, A. Yariv, "Laser phase noise to intensity noise conversion by lowest-order group-velocity dispersion in optical fiber: exact theory," Optics Letters, vol. 25, no. 3, pp. 165-167, Feb. 2000. 
[31] P. Laurencio, M. Medeiros, "Relative intensity noise in optical single side band systems with multiple in line amplifiers: analysis and validation," Fiber and Integrated Optics, vol. 27, pp. 78-88, Mar. 2008.

[32] M. Mohamed, X. Zhang, B. Hraimel, Ke Wu, "Analysis of frequency quadrupling using a single Mach-Zehnder modulator for millimeter-wave generation and distribution over fiber systems," Optics Express, vol. 16, no. 14, Jul. 2008.

[33] European Commission's decision of 21:5:2008 on the harmonization of 3400 3800 $\mathrm{MHz}$ frequency band for terrestrial system capable of providing electronic communication services in the community.

[34] IEEE 802.16 Task Group d, http://www.ieee802.org/16/tgd/

[35] 802.1 In Report, http://www.ieee802.org/11/Reports/tgn_update.html

[36] 802.1 la-1999, High-speed physical layer in the $5 \mathrm{GHz}$ band. 


\section{Appendix A Analysis of Optical Receiver Noise}

Let us assume the optical and electrical filter are rectangular with equivalent bandwidth $B_{o}=\frac{1}{2 \pi} \int_{-\infty}^{\infty}\left|H_{F}(\omega)\right|^{2} d \omega$ and $B_{e}=\frac{1}{2 \pi} \int_{-\infty}^{\infty}\left|H_{e}(\omega)\right|^{2} d \omega$, where $H_{F}(\omega)$ and $H_{e}(\omega)$ are the transfer function of the optical and electrical filter respectively. Because the optical bandwidth is considerably larger than the signal bandwidth, the optical filter won't affect the UWB signal but only the ASE noise. Variance of the ASE-ASE beat noise, $\sigma_{I_{\text {ASE-ASE }}}^{2}(t)$, signal-ASE beat noise, $\sigma_{I_{s_{\text {Igna }-A S E}}^{2}}(t)$, and electrical noise, $\sigma_{e l e c}^{2}$, at the receiver can be expressed as [32]

$$
\begin{aligned}
& \sigma_{I_{\text {ASE }-A S E}}^{2}(t)=2 G_{A} \mathfrak{R}^{2} N_{o}^{2} \frac{1}{2 \pi} \int_{-\infty}^{\infty}\left|H_{e}(\omega)\right|^{2}\left(\left|H_{F}(\omega)\right|^{2} \otimes\left|H_{F}(\omega)\right|^{2}\right) d \omega=2 G_{A} \mathfrak{R}^{2} N_{o}^{2}\left(2 B_{o} B_{e}-B_{e}^{2}\right), \\
& \sigma_{l_{\text {signal }-A S E}}^{2}(t)=2 G_{A} \mathfrak{R}^{2} \frac{1}{2 \pi} N_{o} \int_{-\infty}^{\infty}\left|H_{F}(\omega)\right|^{2} \times\left|S^{*}(t) e^{j \omega t} \otimes h_{e}(t)\right|^{2} d \omega=2 G_{A} \mathfrak{R}^{2} N_{o} P_{o p t} B_{e},
\end{aligned}
$$

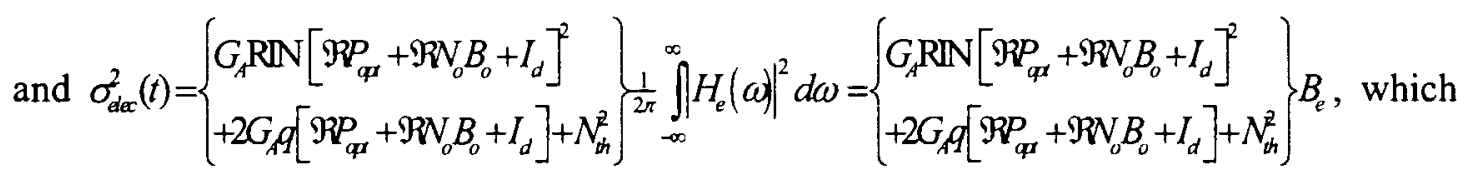
consists of contribution of RIN, $G_{A} \operatorname{RIN}\left[R P_{o p t}+\Re N_{o} B_{o}+I_{d}\right]^{2} B_{e}$, shot noise, $2 G_{A} q\left[\Re P_{o p t}+\Re N_{o} B_{o}+I_{d}\right] B_{e}$, and thermal noise, $N_{t h}^{2} B_{e}$,

where $S^{*}(t)$ is the complex conjugate of the received optical signal at the photodetector, $G_{A}=26 \mathrm{~dB}$ is the RF amplifier gain at the optical receiver, $P_{o p t}$ is the received optical power, and $N_{o}$ represents the single sided ASE noise density for a single polarization expressed by $N_{o}=F h c(G-1) /(2 \lambda), h$ - the Plank's constant, $\lambda$ - the wavelength of the 
laser source, c - speed of light in vacuum, and noise figure of $F=4 \mathrm{~dB}$ and gain of $G=8$ $\mathrm{dB}$ of the EDFA. The symbol $\otimes$ denotes the convolution. $\sigma_{\text {elec }}^{2}$ is the noise contribution from both optical transmitter and receiver electronic noise, and this is called "back-toback" system noise (shot noise, thermal noise, and RIN). $q$ is the electron charge, and $I_{d}=100 \mathrm{nA}$ is the dark current. The variance of thermal noise is given as $N_{t h}^{2}=4 k_{B} T F_{n} B_{e} / R_{L}$, where $k_{B}=1.38 \times 10^{-23} \mathrm{~J} / \mathrm{K}$ is the Boltzman constant, and $F_{n}=5 \mathrm{~dB}$ is noise figure of the RF amplifier at the receiver, $T$ is the room temperature in degree Kelvin, and $R_{L}=50 \Omega$ is the load resistance.

Now, the total noise power will be given as [32]

$$
\sigma_{n}^{2}(t)=\sigma_{I_{\text {ASE-ASE }}}^{2}(t)+2 \sigma_{l_{\text {Sigrat }- \text { ASE }}^{2}}^{2}(t)+\sigma_{\text {elec }}^{2}(t)
$$

All calculated receiver noise contributions are presented in Fig. 17 for the back to back. It is shown that ASE-ASE beat noise is negligible because of the low noise figure of the EDFA and narrow bandwidth of the optical filter used. The output from the RF amplifier at the optical receiver in Fig. 4.1 is directly connected to the data analyzer. The scope captures the signal in time domain and performs Fast Fourier transform (FFT) on it. It analyzes the data within $1.6 \mathrm{GHz}$ bandwidth. The thermal noise within this bandwidth is around $-71.2 \mathrm{dBm}$ which is small compared to other noise contributions. It is seen that shot noise, RIN and signal-ASE beat noise are the major sources of noise at the receiver for higher optical power. 


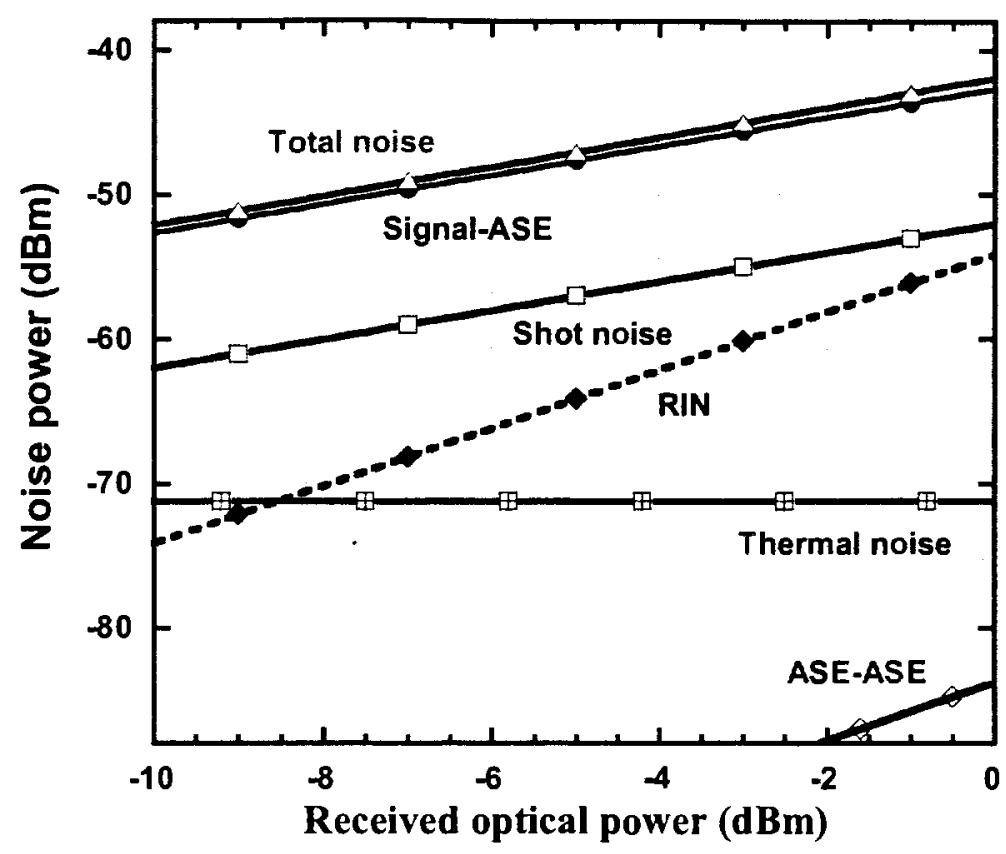

Figure A. 1 Noise power level at the receiver versus received optical power for back-to-back transmission.

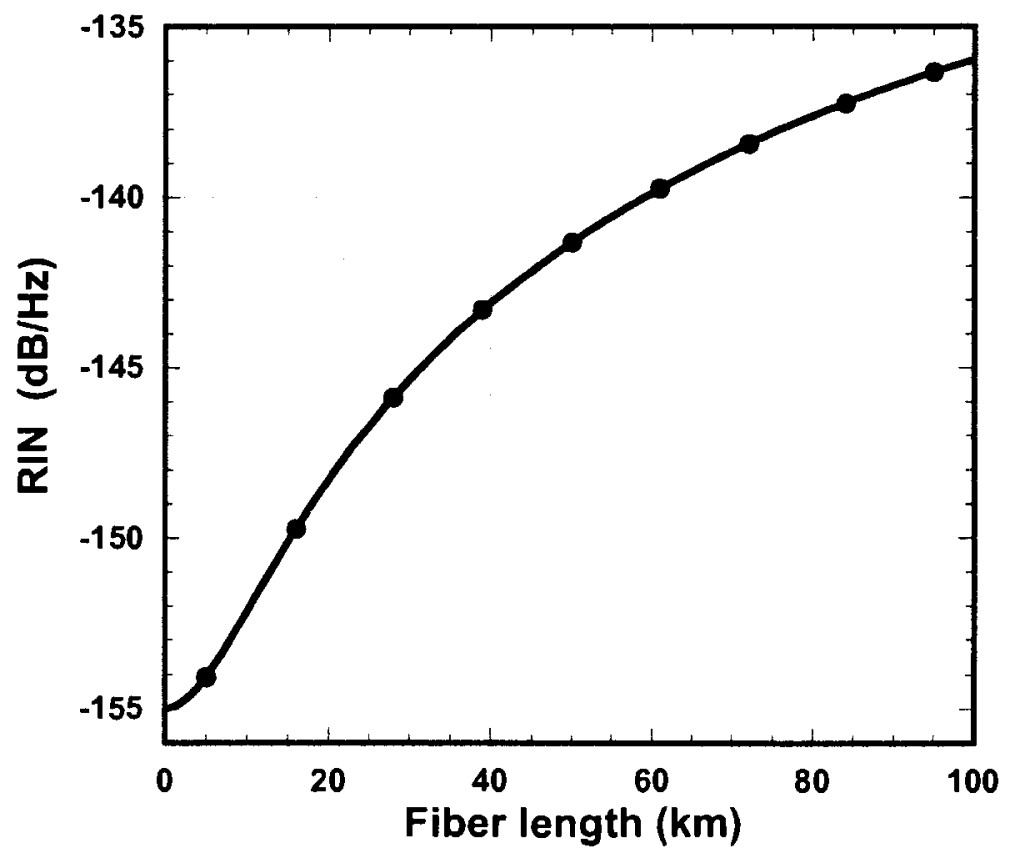

Figure A.2 Calculated total RIN versus fiber length. 
Fiber dispersion changes the laser RIN due phase noise to intensity noise conversion [30-31]. Figure A.2 shows total relative intensity noise due to fiber dispersion for a laser with linewidth of $\Delta \omega /(2 \pi)=800 \mathrm{KHz}$ at $1550 \mathrm{~nm}$ by using [30]

$$
R I N(L) \simeq R I N(0)+\frac{1}{\Omega_{u}-\Omega_{l}} \int_{\Omega_{l}}^{\Omega_{u}} \frac{8 \Delta \omega}{\Omega^{2}} \sin ^{2}\left(\frac{1}{2} \beta_{2} L \Omega^{2}\right) d \Omega
$$

where $\left(\Omega_{u}-\Omega_{l}\right) / 2 \pi=B W$ is the signal occupied bandwidth, $L$ is the fiber length and $R I N(0)=-155 \mathrm{~dB} / \mathrm{Hz}$ is the laser intrinsic $\mathrm{RIN}$.

For the frequency band of 3.176 to $4.744 \mathrm{GHz}$, the dispersion increases the RIN by 6.7 and $11.8 \mathrm{~dB}$ for 20 and $40 \mathrm{~km}$, respectively, compared to the back to back. This is one of the reasons why the EVM degrades with fiber length. 


\section{Appendix B Simulated magnitude and Delay Response of Chebyshev-II Filter}

Simulated magnitude and delay response of Chebyshev-II filter is presented in Fig. B.1 for third, fifth and seventh order filters with different bandwidths. From Fig. B.1(a) we see Chebyshev-II filter has a flat magnitude response in the passband. Though, out of band ripples increase with filter order, which is not of importance if the side-lobe suppression is higher than the required $20 \mathrm{~dB}$ ACPR for WiMedia standard. Fig. B.1(b) shows that the delay increases if the filter order is increased and decreases if the filter bandwidth is increased. Also higher order filters have high delay overshoot at the edges.

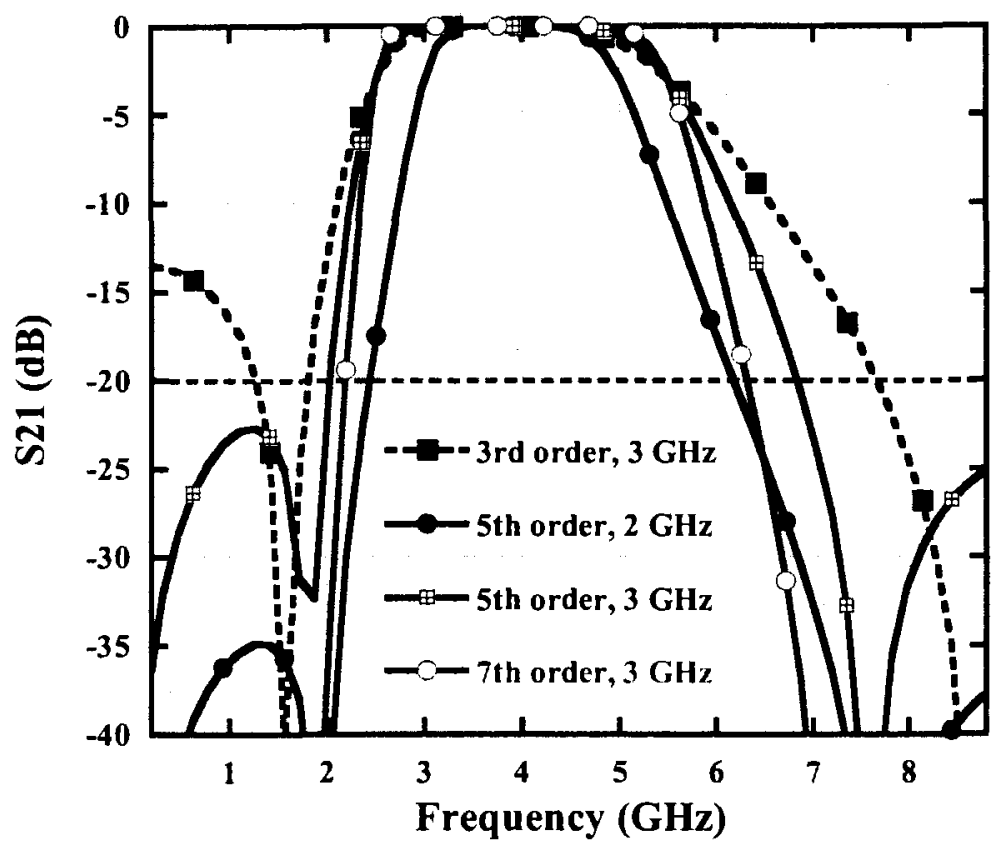

(a) 


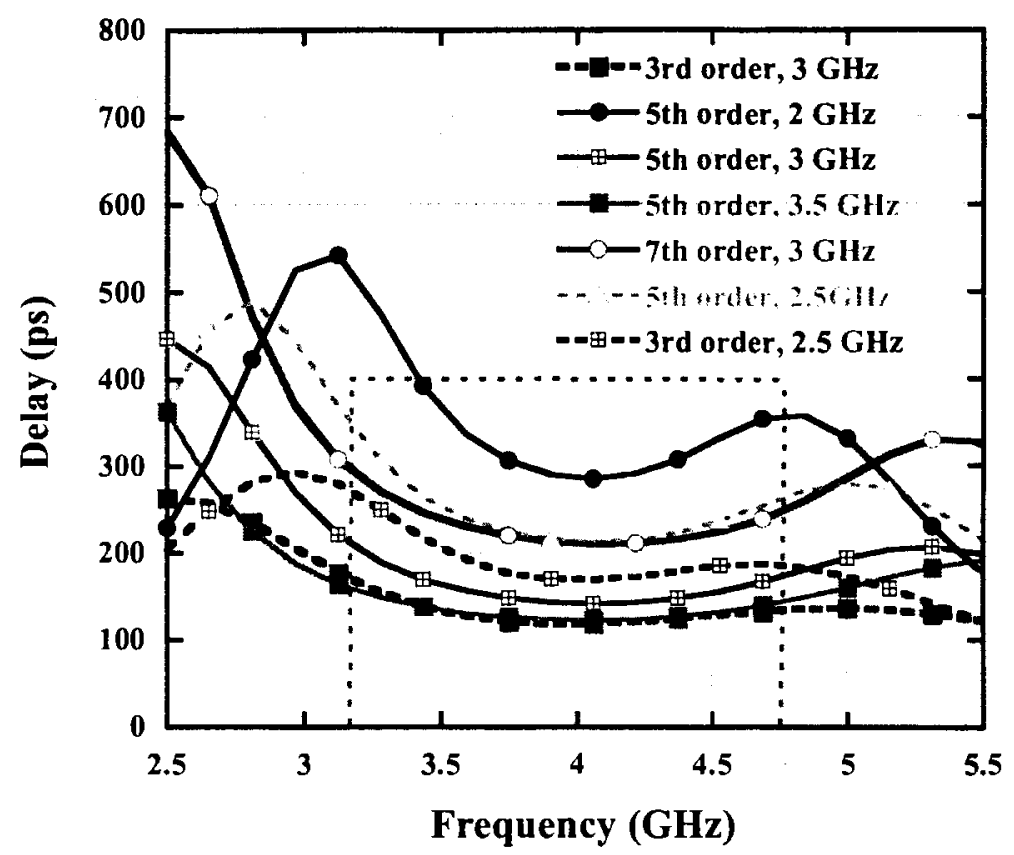

(b)

Figure B.1 Simulated (a) magnitude and (b) delay response of Chebyshev-II filter. 